TRANSACTIONS OF THE

AMERICAN MATHEMATICAL SOCIETY

Volume 349, Number 4, April 1997, Pages 1285-1332

S 0002-9947(97)01805-9

\title{
SOME COMBINATORIAL ASPECTS OF REDUCED WORDS IN FINITE COXETER GROUPS
}

\author{
JOHN R. STEMBRIDGE
}

\begin{abstract}
We analyze the structure of reduced expressions in the Coxeter groups $A_{n}, B_{n}$ and $D_{n}$. Several special classes of elements are singled out for their connections with symmetric functions or the theory of $P$-partitions. Membership in these special classes is characterized in a variety of ways, including forbidden patterns, forbidden subwords, and by the form of canonically chosen reduced words.
\end{abstract}

\section{INTRODUCTION}

This paper is the third in a series on $P$-partitions, symmetric functions, commutation monoids, pattern avoidance, and reduced words in Coxeter groups.

Previously, in [Ste1] we introduced the notion of enriched $P$-partitions. These are related to Schur's $Q$-functions in the same way that Stanley's $P$-partitions (see [St2, $\S 4.5]$ ) are related to Schur's $S$-functions. For example, the generating function $\Delta(P)$ for the set of enriched $P$-partitions of a (labeled) poset $P$ is a quasisymmetric formal series in a set of variables $z_{1}, z_{2}, \ldots$; in case $P$ is a shifted Young diagram, $\Delta(P)$ is a Schur $Q$-function.

In [Ste2], we analyzed the fully commutative elements of Coxeter groups. These are elements $w$ with the property that any reduced expression for $w$ can be obtained from any other by transposing adjacent pairs of commuting generators. One of the characterizing properties of full commutativity is that the reduced words for such an element can be viewed as the linear extensions of a "heap" - a poset whose vertices are labeled by generators of the Coxeter group.

In the present paper, we show that enriched $P$-partitions are closely related to the symmetric functions associated with elements of the Coxeter groups $B_{n}$ and $D_{n}$ (known elsewhere as "stable Schubert polynomials" or "Stanley symmetric functions" - see [BH], [FK1, FK2], [L]). In fact each of these symmetric functions is a linear combination of the generating functions $\Delta(P)$ for certain labeled posets $P$. (See Propositions 6.5 and 8.1.)

This connection has interesting implications for an open problem identified in [Ste1]: the classification of labeled posets $P$ such that $\Delta(P)$ is a symmetric function. There is exactly one term in the $\Delta$-expansion for the symmetric function indexed by a given $w \in B_{n}$ or $D_{n}$ if and only if $w$ is fully commutative. Furthermore, the one labeled poset $P$ that appears in the expansion is the heap. Thus as a

Received by the editors January 1, 1996

1991 Mathematics Subject Classification. Primary 05E05, 05E10, 06A07, 20 F55.

Partially supported by NSF Grants DMS-9057192 and DMS-9401575.

(C)1997 American Mathematical Society 
corollary, we obtain that the heap of any fully commutative member of $B_{n}$ or $D_{n}$ is $\Delta$-symmetric.

We analyze in detail the structure of the fully commutative members of $B_{n}$ and $D_{n}$ and their heaps in Sections 5, 6, and 10. It turns out that for both groups, the fully commutative elements can be naturally partitioned into two families. In one family, the heaps are merely shifted (skew) diagrams, and the corresponding generating functions are (skew) Schur $Q$-functions. On the other hand, although the members of the second family are indexed in a natural way by skew shapes, the corresponding heaps are not Young diagrams. (See Propositions 6.4 and 10.6, and the examples in Figures 2 and 4.)

We also introduce here two additional families of symmetric functions associated with Coxeter groups, one indexed by members of $A_{n}$ and depending on a free parameter $t$, and the second being indexed by a subset of $D_{n}$ and depending on two free parameters. The first family is related to, but not a specialization of, the symmetric functions indexed by $A_{n}$ defined by Stanley in [St1]. Stanley's symmetric functions encode information about the number of reduced words for each $w \in A_{n}$, whereas these new symmetric functions carry information about a generating function for such words in which the number of occurrences of an "endnode" generator is marked. We prove that these new symmetric functions are sums of the symmetric functions associated with $B_{n}$ (see Theorem 3.4). It follows that the number of reduced words for any $w \in A_{n}$ in which an end-node generator occurs $k$ times can be expressed in terms of the number of standard shifted tableaux of certain shapes.

There are four special subsets of $B_{n}$, and six subsets of $D_{n}$, that occur naturally in the course of this work (e.g., the sets of fully commutative members of both $B_{n}$ and $D_{n}$ ). In each of these ten cases, we provide (typically) three characterizations for membership of an element $w$ in the set: a collection of subwords that cannot appear in any reduced word for $w$, a set of "patterns" that must be avoided in a vector representation of $w$, and a set of properties that a canonically chosen reduced word for $w$ must possess. (The latter facilitates enumeration of the members of the set.) The existence of pattern avoidance characterizations for these sets is not surprising, since there are numerous instances of pattern-avoidance arising naturally in previous work on reduced words in $A_{n}$. For example, the 321 -avoiding permutations of $n$ objects are known to be the fully commutative members of $A_{n-1}$ (see [BJS, $\S 2]$ ). On the other hand, two of our ten subsets cannot be given pattern-avoidance characterizations.

\section{Preliminaries}

Let $W$ be a Coxeter group with generating set $S=\left\{s_{i}: i \in I\right\}$, where $I$ is any suitable (finite, totally ordered) index set. For $i, j \in I$, define $m(i, j)$ to be the order of $s_{i} s_{j}$ in $W$, so that $M=[m(i, j)]_{i, j \in I}$ is the Coxeter matrix. One allows $m(i, j)=\infty$.

1.1 Reduced words. Let $I^{*}$ denote the free monoid consisting of all words that can be formed from the alphabet $I$. By a subword of $\mathbf{i}=i_{1} \cdots i_{l} \in I^{*}$, we shall mean a subsequence of $\mathbf{i}$ occupying consecutive positions.

For $w \in W$, let $\ell(w)$ denote the common length of every reduced (i.e., minimal) expression $w=s_{i_{1}} \cdots s_{i_{l}}$ with $i_{1}, \ldots, i_{l} \in I$. The corresponding index sequence $\mathbf{i}=i_{1} \cdots i_{l} \in I^{*}$ is called a reduced word. We use the notation $\mathcal{R}(w)$ for the set of 
reduced words for $w$, and $\mathcal{R}(W)=\bigcup_{w \in W} \mathcal{R}(w)$ for the set of reduced words for all members of $W$.

For integers $m \geq 0$ and $i, j \in I$, define

$$
\langle i, j\rangle_{m}:=\underbrace{i j i j i \cdots}_{m} \in I^{*},
$$

and let $\approx$ denote the congruence on $I^{*}$ generated by the braid relations

$$
\langle i, j\rangle_{m(i, j)} \approx\langle j, i\rangle_{m(i, j)}
$$

for all $i, j \in I$ such that $m(i, j)<\infty$. It is well known that $\mathcal{R}(w)$ constitutes a single braid equivalence class; i.e., any reduced word for $w$ can be obtained from any other by means of the braid relations [B, §IV.1.5].

1.2 Heaps and commutativity classes. Let $\sim$ denote the congruence on $I^{*}$ generated by the braid relations corresponding to pairs of commuting generators of $W$; i.e., $i j \sim j i$ for all $i, j \in I$ such that $m(i, j)=2$. The equivalence class of a word $\mathbf{i} \in I^{*}$ with respect to $\sim$ is called the commutativity class of $\mathbf{i}$. Since $\sim$ is consistent with $\approx$, it follows that for each $w \in W$, there is a decomposition

$$
\mathcal{R}(w)=\mathcal{C}_{1} \dot{\cup} \ldots \dot{\cup} \mathcal{C}_{l},
$$

where each $\mathcal{C}_{i}$ is a commutativity class. In case $\mathcal{R}(w)$ consists of a single commutativity class, we say that $w$ is fully commutative. It is not hard to show that $w$ is fully commutative if and only if $\langle i, j\rangle_{m}$ is not a subword of any $\mathbf{i} \in \mathcal{R}(w)$ whenever $m=m(i, j) \geq 3$.

Given a word $\mathbf{i}=i_{1} \cdots i_{l} \in I^{*}$, the heap of $\mathbf{i}$ is defined to be the partial ordering $P=P_{\mathbf{i}}$ of $\{1, \ldots, l\}$ generated by the transitive closure of the relations

$$
r<_{P} s \text { for } r<s \text { such that } i_{r} i_{s} \nsim i_{s} i_{r} \text { or } i_{r}=i_{s} .
$$

Let $\mathcal{L}\left(P_{\mathbf{i}}\right) \subset I^{*}$ denote the set of (labeled) linear extensions of $P_{\mathbf{i}}$. By this we mean the set of words $i_{\pi(1)} \cdots i_{\pi(l)}$, where $\pi$ ranges over all permutations of $\{1, \ldots, l\}$ consistent with $P_{\mathbf{i}}$ (i.e., $\pi(r)<_{P} \pi(s) \Rightarrow r<s$ ).

The following result is a standard part of the Cartier-Foata theory of commutation monoids. For a proof, see [Ste2, $§ 1.2]$ or Exercise 3.48(b) of [St2].

Proposition 1.1. $\mathcal{L}\left(P_{\mathbf{i}}\right)$ is the commutativity class of $\mathbf{i}$.

It follows that if $w$ is fully commutative, then $\mathcal{R}(w)$ consists of the linear extensions of some labeled poset, namely, the heap of any member of $\mathcal{R}(w)$.

1.3 Canonical factorizations. For $J \subseteq I$, let $W_{J}$ denote the parabolic subgroup of $W$ generated by $\left\{s_{j}: j \in J\right\}$, and define

$$
W^{J}:=\left\{w \in W: j \in J \Rightarrow \ell\left(w s_{j}\right)>\ell(w)\right\} .
$$

It is well-known that $W^{J}$ is a set of (shortest) left coset representatives for $W / W_{J}$. Furthermore, one has $\ell(x y)=\ell(x)+\ell(y)$ for all $x \in W^{J}$ and $y \in W_{J}$ (e.g., [H, $\S 1.10])$.

Assuming $W$ has rank $n$, let us fix a chain $\{1\}=W_{0} \subset W_{1} \subset \cdots \subset W_{n}=W$ of Coxeter groups in which $W_{i-1}$ is a maximal (proper) parabolic subgroup of $W_{i}$. Let $W^{\langle i\rangle}$ denote the set of shortest coset representatives for $W_{i} / W_{i-1}$. In these terms, every $w \in W$ has a unique factorization

$$
w=w_{n} w_{n-1} \cdots w_{1}
$$


with $w_{i} \in W^{\langle i\rangle}$. Moreover, $\ell(w)=\ell\left(w_{1}\right)+\cdots+\ell\left(w_{n}\right)$. We call this the canonical factorization of $w$.

For the classical Weyl groups $A_{n}, B_{n}$ and $D_{n}$, it is possible to choose the subgroup chain so that every member of $W^{\langle i\rangle}$ has a unique reduced word (with mild exceptions in the case of $D_{n}$ - see the beginning of Part II). Thus in these cases, we have not only the notion of a canonical factorization, but also a canonical reduced word for each $w \in W$.

For example, consider $W=A_{n}$ with the index set $I=\{1, \ldots, n\}$ arranged in the usual way so that $m(i, i+1)=3$. Using the subgroup chain $W_{i}=A_{i}$, we obtain

$$
W^{\langle i\rangle}=\left\{1, s_{i}, s_{i-1} s_{i}, \ldots, s_{1} \cdots s_{i}\right\} .
$$

Using $[i, j]$ (for $i \leq j$ ) as an abbreviation for the word $i \cdot(i+1) \cdots j \in I^{*}$, it follows that the canonical reduced words for the members of $A_{n}$ are of the form

$$
\left[m_{1}, n_{1}\right] \cdot\left[m_{2}, n_{2}\right] \cdots\left[m_{r}, n_{r}\right],
$$

where $n \geq n_{1}>\cdots>n_{r} \geq 1$ and $n_{i} \geq m_{i} \geq 1$. It can be shown that the canonical reduced word for each $w \in A_{n}$ is also the first in reverse (i.e., from the right) lexicographic order among the members of $\mathcal{R}(w)$ (see Theorem 2.3 of $[\mathrm{E}]$ ).

1.4 One-line forms. Consider a geometric representation of $W$ as a group generated by reflections acting on $\mathbf{R}^{n}$, with $\langle\cdot, \cdot\rangle$ denoting the associated $W$-invariant symmetric bilinear form. Let $\alpha_{i} \in \mathbf{R}^{n}$ denote the simple root corresponding to $s_{i}$, and fix a vector $\delta \in \mathbf{R}^{n}$ in the interior of the fundamental chamber (i.e., $\left\langle\delta, \alpha_{i}\right\rangle>0$ for all $i \in I$ ). The stabilizer of such a vector is trivial, so one can label the members of $W$ by the vectors in the $W$-orbit of $\delta$.

In these terms, if $\gamma$ is the "label" of $w \in W$ (i.e., $\gamma=w \delta$ ), then (cf. [H, §5.4])

$$
\ell\left(s_{i} w\right)>\ell(w) \Leftrightarrow\left\langle\gamma, \alpha_{i}\right\rangle>0 .
$$

For example, consider $W=A_{n-1}$. We can represent $W$ acting on $\mathbf{R}^{n}$ with a Euclidean inner product. Using $\varepsilon_{1}, \ldots, \varepsilon_{n}$ to denote the standard orthonormal basis of $\mathbf{R}^{n}$, we can choose the simple roots to be $\alpha_{i}=\varepsilon_{i+1}-\varepsilon_{i}(1 \leq i<n)$, and take $\delta:=(1,2, \ldots, n)=\varepsilon_{1}+2 \varepsilon_{2}+\cdots+n \varepsilon_{n}$. The orbit of $\delta$ consists of all permutations of $(1,2, \ldots, n)$. Thus our convention of using vectors to label Coxeter group elements amounts to a generalization of the usual one-line description of a permutation. However, there is one significant difference. By our convention, $(2,3,1)$ labels the (unique) permutation that maps $\varepsilon_{1}+2 \varepsilon_{2}+3 \varepsilon_{3}$ to $2 \varepsilon_{1}+3 \varepsilon_{2}+\varepsilon_{3}$ (i.e., $\varepsilon_{1} \mapsto \varepsilon_{3}, \varepsilon_{2} \mapsto \varepsilon_{1}, \varepsilon_{3} \mapsto \varepsilon_{2}$ ), whereas the more common convention is to use the inverse; i.e., $(2,3,1)$ labels the permutation $\varepsilon_{1} \mapsto \varepsilon_{2}, \varepsilon_{2} \mapsto \varepsilon_{3}, \varepsilon_{3} \mapsto \varepsilon_{1}$. Thus if $\left(w_{1}, \ldots, w_{n}\right)$ is the vector label of $w \in A_{n-1}$, then by our convention, the vector label of $s_{i} w$ is obtained by interchanging $w_{i}$ and $w_{i+1}$. Those using the inverse convention would interchange the occurrences of $i$ and $i+1$.

Given that $\delta$ and the choice of basis is understood, we will refer to the coordinate sequence for the vector label of a given $w \in W$ as the one-line form of $w$.

1.5 Dominance. Suppose that $W^{\prime}$ is a second Coxeter group, with generating set $S^{\prime}=\left\{s_{i}^{\prime}: i \in I\right\}$ and Coxeter matrix $M^{\prime}=\left[m^{\prime}(i, j)\right]_{i, j \in I}$. Note that by using $I$ as the index set for $S$ and $S^{\prime}$, we are presupposing that $W$ and $W^{\prime}$ have the same rank. Under these conditions, we say that $W$ dominates $W^{\prime}$ if $m(i, j) \geq m^{\prime}(i, j)$ for all $i, j \in I$.

For example, $B_{n}$ dominates $A_{n}$ and $A_{m+n}$ dominates $A_{m} \times A_{n}$. 
Proposition 1.2. If $W$ dominates $W^{\prime}$, then $\mathcal{R}\left(W^{\prime}\right) \subseteq \mathcal{R}(W)$. Furthermore, if $w \in W$ and $\mathcal{R}(w) \cap \mathcal{R}\left(W^{\prime}\right) \neq \varnothing$, then

(a) Any $\mathbf{i} \in \mathcal{R}(w)$ can be transformed into any $\mathbf{j} \in \mathcal{R}(w)$ via braid relations involving only those pairs $i, j \in I$ such that $m(i, j)=m^{\prime}(i, j)$.

(b) $\mathcal{R}(w) \subseteq \mathcal{R}\left(w^{\prime}\right)$ for some $w^{\prime} \in W^{\prime}$.

Proof. To prove $\mathcal{R}\left(W^{\prime}\right) \subseteq \mathcal{R}(W)$, suppose $\mathbf{i}=i_{1} \cdots i_{l} \notin \mathcal{R}(W)$. Then there must exist some $k>1$ such that $i_{1} \cdots i_{k-1}$ is $W$-reduced and $i_{1} \cdots i_{k}$ is not. Hence some member of the $W$-braid equivalence class of $i_{1} \cdots i_{k-1}$ ends with $i_{k}$, and thus some word $\mathbf{j}$ with two equal consecutive letters (both equal to $i_{k}$ ) is $W$-braid equivalent to $\mathbf{i}$.

Now consider any sequence of $W$-braid relations that transform $\mathbf{i}$ into $\mathbf{j}$. If these relations only involve pairs $i, j \in I$ such that $m(i, j)=m^{\prime}(i, j)$, then this sequence is equally valid as a series of $W^{\prime}$-braid relations, thus proving $\mathbf{i} \notin \mathcal{R}\left(W^{\prime}\right)$. Otherwise, immediately prior to the first time a $W$-braid relation is applied in which $m(i, j)>m^{\prime}(i, j)$, we will have a word that is $W^{\prime}$-braid equivalent to $\mathbf{i}$ containing $\langle i, j\rangle_{m}$ as a subword for some $m>m^{\prime}=m^{\prime}(i, j)$. However, if the $W^{\prime}$-braid relation $\langle i, j\rangle_{m^{\prime}} \approx\langle j, i\rangle_{m^{\prime}}$ is applied at the beginning of $\langle i, j\rangle_{m}$, one obtains two equal consecutive letters, thereby proving $\mathbf{i} \notin \mathcal{R}\left(W^{\prime}\right)$.

To prove (a) and (b), suppose that $\mathbf{i} \in \mathcal{R}(w)$ is $W^{\prime}$-reduced. Any $W$-braid transformations of $\mathbf{i}$ involving pairs $i, j \in I$ such that $m(i, j)=m^{\prime}(i, j)$ are also valid in $W^{\prime}$ and hence generate words that are also $W^{\prime}$-reduced. In particular, none of these words can contain $\langle i, j\rangle_{m}$ as a subword for any $i, j \in I$ and $m>m^{\prime}(i, j)$. And hence they constitute the full $W$-braid equivalence class of $\mathbf{i}$, since there are no opportunities among these words to apply any of the other $W$-braid relations. Since these words are also $W^{\prime}$-equivalent, they must belong to $\mathcal{R}\left(w^{\prime}\right)$ for some $w^{\prime} \in W^{\prime}$.

Remark 1.3. (a) For specific dominating pairs $W$ and $W^{\prime}$, it is an interesting problem to explicitly determine the set $X=\left\{w \in W: \mathcal{R}(w) \subset \mathcal{R}\left(W^{\prime}\right)\right\}$ of $W^{\prime}$-reduced members of $W$ and the (unique) partition of $X$ into subsets $X\left(w^{\prime}\right)$ indexed by $w^{\prime} \in W^{\prime}$ such that

$$
\mathcal{R}\left(w^{\prime}\right)=\bigcup_{w \in X\left(w^{\prime}\right)} \mathcal{R}(w)
$$

In Section 2 we will treat the case $\left(W, W^{\prime}\right)=\left(B_{n}, A_{n}\right)$ in detail.

(b) It is tempting to guess that $w \in W$ is $W^{\prime}$-reduced if and only if for all $m>m^{\prime}(i, j),\langle i, j\rangle_{m}$ does not occur as a subword of any $\mathbf{i} \in \mathcal{R}(w)$. Although this condition is clearly necessary, it is not sufficient in general. For example, see Theorem 2.3.

1.6 Quasi-symmetric functions. Following the notation of [Ste1], let $\boldsymbol{\Sigma}=$ $\bigoplus_{l \geq 0} \Sigma^{l}$ denote the graded ring of quasi-symmetric functions in the variables $z_{1}, z_{2}, \ldots$, with integer coefficients. Given any twin-free word $\mathbf{i}=i_{1} \cdots i_{l} \in I^{*}$ (i.e., distinct adjacent letters), we define

$$
L_{\mathbf{i}}:=\sum_{\substack{j_{1} \leq \cdots \leq j_{l} \\ j_{k}=j_{k+1} \Rightarrow i_{k}<i_{k+1}}} z_{j_{1}} \cdots z_{j_{l}} \in \mathbf{\Sigma}^{l},
$$

bearing in mind that $I$ is assumed to be totally ordered. Clearly $L_{\mathbf{i}}$ depends only on $l$ and the descent set $D(\mathbf{i}):=\left\{1 \leq k<l: i_{k}>i_{k+1}\right\}$; thus we may write 
$L_{D}$ for $L_{\mathbf{i}}$ whenever $D=D(\mathbf{i})$. It is not hard to show that the set of $L_{D}$ 's for $D \subseteq\{1, \ldots, l-1\}$ freely generate $\boldsymbol{\Sigma}^{l}$ as a $\mathbf{Z}$-module.

We will also be making use of a second family of quasi-symmetric functions indexed by twin-free words. For this family, totally order the nonzero integers so that

$$
-1 \prec+1 \prec-2 \prec+2 \prec-3 \prec+3 \prec \cdots .
$$

Letting the indices $j_{1}, \ldots, j_{l}$ range over nonzero integers, we define

$$
K_{\mathbf{i}}:=\sum_{\substack{j_{1} \preccurlyeq \cdots \preccurlyeq j_{l} \\ j_{k}=j_{k+1}>0 \Rightarrow i_{k}<i_{k+1} \\ j_{k}=j_{k+1}<0 \Rightarrow i_{k}>i_{k+1}}} z_{\left|j_{1}\right|} \cdots z_{\left|j_{l}\right|}
$$

for any twin-free $\mathbf{i}=i_{1} \cdots i_{l}$. Again it is clear that $K_{\mathbf{i}}$ depends only on $l$ and the descent set $D(\mathbf{i})$. Less clear, but true (see Proposition 2.2 of [Ste1]), is the fact that $K_{\mathbf{i}}$ depends only on $l$ and the peak set

$$
\Lambda(\mathbf{i}):=\left\{1<k<l: i_{k-1}<i_{k}>i_{k+1}\right\} .
$$

Thus we may write $K_{\Lambda}$ for $K_{\mathbf{i}}$ whenever $\Lambda=\Lambda(\mathbf{i})$. The $K_{\Lambda}$ 's freely generate a $\mathbf{Z}$-submodule $\boldsymbol{\Pi}^{l}$ of $\boldsymbol{\Sigma}^{l}$; in fact, $\boldsymbol{\Pi}=\bigoplus_{l \geq 0} \boldsymbol{\Pi}^{l}$ is a graded subring of $\boldsymbol{\Sigma}$ (see Theorem 3.1 of [Ste1]).

Now let $U$ be any ring with unity. Adjoining central indeterminates $z, z_{1}, z_{2}, \ldots$, if $F(z) \in U[z]$ satisfies $F(0)=1$, then $F\left(z_{1}\right) F\left(z_{2}\right) \cdots$ is quasi-symmetric. More precisely, working in the ring $\mathbf{Z}\left[\left[z_{1}, z_{2}, \ldots\right]\right] \otimes U$, it is clear that $F\left(z_{1}\right) F\left(z_{2}\right) \cdots \in$ $\boldsymbol{\Sigma} \otimes U$. We will be concerned with expansions of $F\left(z_{1}\right) F\left(z_{2}\right) \cdots$ in some particular cases; namely,

$$
\begin{aligned}
& F_{n}^{+}(z):=\left(1+z u_{1}\right)\left(1+z u_{2}\right) \cdots\left(1+z u_{n}\right), \\
& F_{n}^{-}(z):=\left(1+z u_{n}\right) \cdots\left(1+z u_{2}\right)\left(1+z u_{1}\right), \\
& G_{n}(z):=F_{n}^{-}(z) F_{n}^{+}(z),
\end{aligned}
$$

where $u_{1}, \ldots, u_{n} \in U$ satisfy $u_{1}^{2}=\cdots=u_{n}^{2}=0$.

Proposition 1.4. Let $I=\{1,2, \ldots, n\}$. We have

(a) $F_{n}^{+}\left(z_{1}\right) F_{n}^{+}\left(z_{2}\right) \cdots=\sum_{\mathbf{i} \in I^{*}} L_{D(\mathbf{i})} u_{\mathbf{i}}$,

(b) $G_{n}\left(z_{1}\right) G_{n}\left(z_{2}\right) \cdots=\sum_{\mathbf{i} \in I^{*}} K_{\Lambda(\mathbf{i})} u_{\mathbf{i}}$,

where $u_{\mathbf{i}}:=u_{i_{1}} \cdots u_{i_{l}}$ if $\mathbf{i}=i_{1} \cdots i_{l}$.

Proof. We prove (b), leaving (a) to the reader.

For (b), selecting a term from the expansion of $G_{n}\left(z_{1}\right) G_{n}\left(z_{2}\right) \cdots$ can be encoded by an ordered sequence of the form $\left(i_{1}, j_{1}\right), \ldots,\left(i_{l}, j_{l}\right)$; the presence of $(i,-j)$ (resp., $(i,+j))$ indicates selection of the term $z_{j} u_{i}$ from the first (resp., second) of the two occurrences of the binomial $\left(1+z_{j} u_{i}\right)$. We may assume that $\mathbf{i}=i_{1} \cdots i_{l}$ is twinfree; otherwise $u_{\mathbf{i}}=0$. The possible selection sequences are characterized by the properties

$$
j_{1} \preccurlyeq \cdots \preccurlyeq j_{l}, \quad j_{k}=j_{k+1}>0 \Rightarrow i_{k}<i_{k+1}, \quad j_{k}=j_{k+1}<0 \Rightarrow i_{k}>i_{k+1},
$$

so for a fixed choice of $\mathbf{i}$, the net contribution of these selections is $K_{\mathbf{i}} u_{\mathbf{i}}$. 
1.7 Nil Coxeter rings. Specializing the setting of the previous subsection, let $U$ be the nil Coxeter ring associated with $W$. That is, let $U$ be the free associative ring with unity generated by $u_{i}: i \in I$, modulo the relations

$$
u_{i}^{2}=0, \quad\left\langle u_{i}, u_{j}\right\rangle_{m(i, j)}=\left\langle u_{j}, u_{i}\right\rangle_{m(i, j)} \quad(m(i, j)<\infty) .
$$

For $w \in W$, define $u_{w}=u_{\mathbf{i}}$ for any $\mathbf{i} \in \mathcal{R}(w)$; the result is clearly independent of the choice of $\mathbf{i}$, and it is not hard to show that $\left\{u_{w}: w \in W\right\}$ is a free $\mathbf{Z}$-basis of $U$.

Nil Coxeter rings have been used to great advantage by Fomin-Stanley [FS] and Fomin-Kirillov [FK1, FK2] in analyzing various symmetric functions associated with Coxeter groups. For example, consider $W=A_{n}$. In [St1], Stanley defined quasi-symmetric functions for each $w \in A_{n}$ by setting

$$
F_{A}(w):=\sum_{\mathbf{i} \in \mathcal{R}(w)} L_{D(\mathbf{i})},
$$

and used these to derive numerous combinatorial properties of reduced words in $A_{n}$. A crucial feature of these formal series, not obvious from their definition, is the fact that they are symmetric in the variables $z_{1}, z_{2}, \ldots$

By Proposition 1.4(a), we see that

$$
F_{n}^{+}\left(z_{1}\right) F_{n}^{+}\left(z_{2}\right) \cdots=\sum_{w \in A_{n}} F_{A}(w) u_{w}
$$

That is, $F_{A}(w)$ is the coefficient of $u_{w}$ in $F_{n}^{+}\left(z_{1}\right) F_{n}^{+}\left(z_{2}\right) \ldots$. The symmetry of $F_{A}(w)$ is therefore a corollary of the following lemma due to Fomin-Stanley [FS]. (We include below a slightly different proof.)

Lemma 1.5. We have

(a) $F_{n}^{+}(x) F_{n}^{+}(y)=F_{n}^{+}(y) F_{n}^{+}(x)$.

(b) $F_{n}^{-}(x) F_{n}^{+}(y)=F_{n}^{+}(y) F_{n}^{-}(x)$.

Proof. Since $F_{n}^{+}(-x) F_{n}^{-}(x)=1$, it suffices to prove (b). Proceeding by induction on $n$, leaving the basis of the induction $(n \leq 2)$ to the reader, we find

$$
\begin{aligned}
F_{n}^{+}(y) F_{n}^{-}(x) & =F_{n-2}^{+}(y)\left(1+y u_{n-1}\right)\left(1+y u_{n}\right) \cdot\left(1+x u_{n}\right)\left(1+x u_{n-1}\right) F_{n-2}^{-}(x) \\
& =F_{n-2}^{+}(y)\left(1+x u_{n}\right)\left(1+x u_{n-1}\right) \cdot\left(1+y u_{n-1}\right)\left(1+y u_{n}\right) F_{n-2}^{-}(x) \\
& =\left(1+x u_{n}\right) F_{n-2}^{+}(y)\left(1+(x+y) u_{n-1}\right) F_{n-2}^{-}(x)\left(1+y u_{n}\right) \\
& =\left(1+x u_{n}\right) F_{n-1}^{+}(y) F_{n-1}^{-}(x)\left(1+y u_{n}\right) \\
& =\left(1+x u_{n}\right) F_{n-1}^{-}(x) F_{n-1}^{+}(y)\left(1+y u_{n}\right)=F_{n}^{-}(x) F_{n}^{+}(y) .
\end{aligned}
$$

The second and fifth equalities are instances of the induction hypothesis.

Remark 1.6. A second corollary of Lemma 1.5 is that $G_{n}(x)$ commutes with $G_{n}(y)$, so the coefficient of $u_{w}$ in $G_{n}\left(z_{1}\right) G_{n}\left(z_{2}\right) \cdots$ is a symmetric function $G_{A}(w)$. Furthermore, by Proposition 1.4(b), we have

$$
G_{A}(w)=\sum_{\mathbf{i} \in \mathcal{R}(w)} K_{\Lambda(\mathbf{i})} .
$$

However, one can show that $G_{A}(w)$ is merely a "diagonal superfication" of $F_{A}(w)$ (i.e., the image of $F_{A}(w)$ under the map $\theta$ that kills even power sums and doubles odd power sums - see [Ste1, $\S 3])$. Hence $G_{A}(w)$ does not carry more information 
than $F_{A}(w)$ itself. However in Section 3, we will consider a one-parameter refinement of $G_{A}(w)$ that encodes combinatorial information about $\mathcal{R}(w)$ not carried by $F_{A}(w)$.

Part I. $B_{n}$

Let $s_{0}, s_{1}, \ldots, s_{n-1}$ denote generators for the Coxeter group $B_{n}$, arranging the indices so that $m(0,1)=4$ and $m(i-1, i)=3$ for $1<i<n$. For $w \in B_{n}$, the number of occurrences of 0 in any reduced word for $w$ will be denoted $\ell_{0}(w)$; it is independent of the choice of reduced word since this quantity is preserved by the braid relations.

The shortest left coset representatives for $B_{n} / B_{n-1}$ consist of

$$
\begin{aligned}
\left\{1, s_{n-1}, s_{n-2} s_{n-1}, \ldots, s_{0} s_{1} \cdots s_{n-1}, s_{1} s_{0} s_{1} \cdots s_{n-1}\right. & \\
& \left.\ldots, s_{n-1} \cdots s_{1} s_{0} s_{1} \cdots s_{n-1}\right\} .
\end{aligned}
$$

There is only one reduced word for each of these coset representatives, so every $w \in B_{n}$ has a canonical reduced word, as explained in Section 1.3. Extending the notation of Section 1.3 slightly, for integers $i, j$ such that $0 \leq i \leq j$ we define $[i, j]$ and $[-i, j]$ to be the words $i \cdot(i+1) \cdots j$ and $i \cdot(i-1) \cdots 101 \cdots j$, respectively. In these terms, the canonical reduced words for the members of $B_{n}$ are the expressions

$$
\left[m_{1}, n_{1}\right] \cdot\left[m_{2}, n_{2}\right] \cdots\left[m_{r}, n_{r}\right],
$$

where $n>n_{1}>\cdots>n_{r} \geq 0$ and $\left|m_{i}\right| \leq n_{i}$.

With $\varepsilon_{1}, \ldots, \varepsilon_{n}$ as the standard orthonormal basis of $\mathbf{R}^{n}$, we take $\varepsilon_{i+1}-\varepsilon_{i}$ (resp., $\varepsilon_{1}$ ) as the simple root corresponding to $s_{i}$ for $i \geq 1$ (resp., $i=0$ ). The vector

$$
\delta=\varepsilon_{1}+2 \varepsilon_{2}+\cdots+n \varepsilon_{n}=(1,2, \ldots, n)
$$

belongs to the interior of the fundamental chamber defined by these simple roots, and its orbit consists of all signed permutations of $(1,2, \ldots, n)$. These constitute the one-line forms of the members of $B_{n}$, as explained in Section 1.4.

In the following, we will derive numerous pattern-avoidance characterizations of various subsets of $B_{n}$. While it is possible (however complicated) to give a general definition of pattern avoidance, it is best explained by special cases. For example, an element $w \in B_{n}$ is said to avoid the pattern $(2,-1,-3)$ if in the one-line form of $w$, say $\left(w_{1}, \ldots, w_{n}\right)$, there is no triple $i<j<k$ such that $-w_{k}>w_{i}>-w_{j}>0$.

\section{The $A$-Reduced members of $B_{n}$}

As we noted in Section 1.5, $B_{n}$ dominates $A_{n}$. The following result is a first step towards characterizing when $w \in B_{n}$ is $A$-reduced (i.e., $\mathcal{R}(w) \subset \mathcal{R}\left(A_{n}\right)$ ).

Lemma 2.1. For $w \in B_{n}, 0101$ is a subword of some $\mathbf{i} \in \mathcal{R}(w)$ if and only if the pattern $(-1,-2)$ occurs in the one-line form of $w$.

Proof. Let $\left(w_{1}, \ldots, w_{n}\right)$ be the one-line form of $w$, and suppose that the pattern $(-1,-2)$ occurs in positions $i$ and $j$, so that $i<j$ and $-w_{j}>-w_{i}>0$. Among all such choices for $i$ and $j$, we can choose one that minimizes $j-i$. If there is a positive entry in any position prior to $j$, then we would have $w_{k}>0>w_{k+1}$ for some $k(1 \leq k<j)$. However in that case, $\ell\left(s_{k} w\right)<\ell(w)$ and $s_{k} w$ still contains 
the pattern $(-1,-2)$, so by induction on length, $s_{k} w$ (and hence $w$ ) has a reduced word containing 0101.

Otherwise, every entry prior to $w_{j}$ is negative. In particular, $i$ and $j$ must be consecutive; otherwise, $j-i$ would not be minimal. We also have $\ell\left(s_{0} w\right)<\ell(w)$ since $w_{1}<0$. If $i>1$, then $s_{0} w$ still contains the pattern $(-1,-2)$, so again by induction, $s_{0} w$ (and hence $w$ ) has a reduced word containing 0101 .

The only remaining possibility is that $i=1$ and $j=2$. However since 0101 is a reduced word for the member of $B_{2}$ whose one-line form is $(-1,-2)$, it follows that $w$ has a reduced word that begins with 0101 .

For the converse, it suffices to prove the following.

(i) If $w$ has a reduced word that begins $0101 \ldots$, then $w$ contains the pattern $(-1,-2)$.

(ii) If $w$ contains the pattern $(-1,-2)$ and $\ell\left(s_{j} w\right)>\ell(w)$, then $s_{j} w$ also contains the pattern $(-1,-2)$.

For (i), recall that $0101 \approx 1010$. Thus if $w$ has a reduced word that begins with 0101, then it has reduced words that begin with 0 and 1; i.e., $\ell\left(s_{0} w\right)<\ell(w)$ and $\ell\left(s_{1} w\right)<\ell(w)$. Hence $w_{1}<0$ and $w_{1}>w_{2}$, so $w$ contains the pattern $(-1,-2)$.

For (ii), suppose that $(-1,-2)$ occurs in $w$ and $\ell\left(s_{j} w\right)>\ell(w)$. If $j=0$, then $w_{1}>0$ and $s_{j} w$ has one-line form $\left(-w_{1}, w_{2}, \ldots, w_{n}\right)$. Clearly this can only increase the number of occurrences of $(-1,-2)$. If $j>0$, then $w_{j}<w_{j+1}$, and the one-form of $w$ is obtained by interchanging $w_{j}$ and $w_{j+1}$. If $w_{j}$ and $w_{j+1}$ are both negative, this increases the number of occurrences of $(-1,-2)$; otherwise, the relative positions of the negative entries are unchanged.

Let $s_{0}^{\prime}, s_{1}^{\prime}, \ldots, s_{n-1}^{\prime}$ denote a set of generators for $A_{n}$. We are deliberately using the index set $\{0,1, \ldots, n-1\}$ here so that the dominance relationship between $B_{n}$ and $A_{n}$ remains conspicuous. Since $0101 \approx 1010$ is the only $B_{n}$-braid relation that is not also valid for $A_{n}$, it follows that for the $(-1,-2)$-avoiding elements $w \in B_{n}$, the mapping $s_{i} \mapsto s_{i}^{\prime}$ is well-defined in the sense that

$$
w^{\prime}=s_{i_{1}}^{\prime} \cdots s_{i_{l}}^{\prime} \in A_{n}
$$

is independent of the choice of $\mathbf{i}=i_{1} \cdots i_{l} \in \mathcal{R}(w)$.

To describe this mapping more explicitly, we need to choose coordinates for $A_{n}$. For this we pass to $\mathbf{R}^{n+1}$ and use $\varepsilon_{0}$ as the name for the new coordinate. By convention, we will write $\left(a_{0}, a_{1}, \ldots, a_{n}\right)$ for the vector $a_{0} \varepsilon_{0}+\cdots+a_{n} \varepsilon_{n}$. For the simple root corresponding to $s_{i}^{\prime}$, we choose $\varepsilon_{i+1}-\varepsilon_{i}$. The vector $\delta$ belongs to the fundamental chamber defined by these roots, so we can use its $A_{n}$-orbit (namely, all permutations of $(0,1, \ldots, n))$ as the one-line forms for the members of $A_{n}$.

We now define a "bumping" map $\mathfrak{b}: B_{n} \rightarrow A_{n}$ as follows. Let $\left(w_{1}, \ldots, w_{n}\right)$ be the one-line form of some $w \in B_{n}$, and suppose that $i_{1}<\cdots<i_{k}$ are the positions $i$ such that $w_{i}<0$. Define $\mathfrak{b}(w)$ to be the member of $A_{n}$ whose one-line form is $\left(x_{0}, \ldots, x_{n}\right)$, where

$$
x_{0}=-w_{i_{1}}, \quad x_{i_{1}}=-w_{i_{2}}, \quad \ldots \quad, x_{i_{k-1}}=-w_{i_{k}}, \quad x_{i_{k}}=0,
$$

and $x_{j}=w_{j}$ for $w_{j}>0$. In other words, we insert 0 into $w$ from the right, where it bumps out and changes the sign of the first encountered negative entry, which in turn bumps out and changes the sign of the next negative entry, and so on. The algorithm terminates with the last bumped element stopping at the 0th position. 
For example,

$$
\begin{aligned}
& \text { if } w=(3,-6, \quad 1,-4,-2, \quad 5, \quad 7) \in B_{7}, \\
& \text { then } \mathfrak{b}(w)=(6, \quad 3, \quad 4, \quad 1, \quad 2, \quad 0, \quad 5, \quad 7) \in A_{7} \text {. }
\end{aligned}
$$

Lemma 2.2. If $w \in B_{n}$ avoids $(-1,-2)$ and $\mathbf{i}=i_{1} \cdots i_{l} \in \mathcal{R}(w)$, then

$$
\mathfrak{b}(w)=w^{\prime}=s_{i_{1}}^{\prime} \cdots s_{i_{l}}^{\prime} .
$$

Furthermore, if there is some index $j$ such that $\ell\left(s_{j} w\right)<\ell(w)$ and $\ell\left(s_{j}^{\prime} w^{\prime}\right)>\ell\left(w^{\prime}\right)$, then $w$ contains the pattern $(1,-3,-2)$.

Proof. We prove both assertions by induction on $\ell(w)$. If $\ell(w)=0$ the claims are trivial, so assume $\ell(w)>0$ and choose an index $j$ such that $\ell\left(s_{j} w\right)<\ell(w)$. If $w$ avoids $(-1,-2)$, then 0101 cannot occur in any $\mathbf{i}=i_{1} \cdots i_{l} \in \mathcal{R}(w)$ (Lemma 2.1). It follows that $w^{\prime}=s_{i_{1}}^{\prime} \cdots s_{i_{l}}^{\prime}$ does not depend on the choice of $\mathbf{i}$, and (by the induction hypothesis) $\mathfrak{b}\left(s_{j} w\right)=s_{j}^{\prime} w^{\prime}$. Thus for the first assertion, it suffices to prove that $\mathfrak{b}(w)=s_{j}^{\prime} \mathfrak{b}\left(s_{j} w\right)$.

Case 1: $j=0$. Let $-a<0$ be the first entry in the one-line form of $w$ (this entry is negative since $\left.\ell\left(s_{0} w\right)<\ell(w)\right)$, and let $-b \leq 0$ be the entry that bumps $-a$ when 0 is inserted into $w$. The first two entries in the one-line form of $\mathfrak{b}(w)$ must be $(a, b)$. On the other hand, in $s_{0} w$ the first entry is $a>0$ and $-b$ is the leftmost negative entry (or there are no negative entries, if $b=0$ ). The first two entries of $\mathfrak{b}\left(s_{0} w\right)$ are therefore $(b, a)$, and the remaining entries agree with $\mathfrak{b}(w)$. Hence $\mathfrak{b}(w)=s_{0}^{\prime} \mathfrak{b}\left(s_{0} w\right)=w^{\prime}$, as desired. Also, regarding the second assertion, note that $\ell\left(s_{0}^{\prime} w^{\prime}\right)>\ell\left(w^{\prime}\right)$ occurs only if $a<b$, in which case the pattern $(-1,-2)$ occurs in $w$, a contradiction.

Case 2: $j \geq 1$. Let $a, b$ be the entries in positions $j, j+1$ of the one-line form of $w$; the one-line form of $s_{j} w$ is obtained by interchanging $a$ and $b$. Since $\ell\left(s_{j} w\right)<\ell(w)$, we must have $a>b$, and since $w$ avoids $(-1,-2), a$ and $b$ cannot both be negative. It follows that $\mathfrak{b}$ commutes with permuting $a$ and $b$, and hence $\mathfrak{b}(w)=s_{j}^{\prime} \mathfrak{b}\left(s_{j} w\right)=$ $w^{\prime}$, as desired.

Regarding the second assertion, suppose that $\ell\left(s_{j}^{\prime} w^{\prime}\right)>\ell\left(w^{\prime}\right)$. Since the positive entries of $w$ remain stationary, this can occur only if one of $a$ or $b$ is negative. Since $a>b$, this requires $a>0>b$. If $c \leq 0$ is the entry that bumps $b$ when 0 is inserted into $w$, then we must have $b<c$; otherwise $w$ would contain the pattern $(-1,-2)$. Furthermore, since we then have $a$ and $-c$ in positions $j$ and $j+1$ of $w^{\prime}=\mathfrak{b}(w)$ (respectively), the fact that $\ell\left(s_{j}^{\prime} w^{\prime}\right)>\ell\left(w^{\prime}\right)$ implies $a<-c$. Thus we have $b<c<-a<0$, and the $w$-subsequence $(a, b, c)$ fits the pattern $(1,-3,-2)$.

Theorem 2.3. For $w \in B_{n}$, the following are equivalent.

(a) $w$ is A-reduced (i.e., $\mathcal{R}(w) \subset \mathcal{R}\left(A_{n}\right)$ ).

(b) Neither 0101 nor 1012101 occur as subwords of any $\mathbf{i} \in \mathcal{R}(w)$.

(c) $w$ avoids the patterns $(-1,-2)$ and $(1,-3,-2)$.

Proof. (a) $\Rightarrow(\mathrm{b})$ is immediate since 0101 and 1012101 are not reduced words for $A_{n}$.

(b) $\Rightarrow(\mathrm{c})$. If the one-line form of $w$, say $\left(w_{1}, \ldots, w_{n}\right)$, contains the pattern $(-1,-2)$, then 0101 must occur as a subword of some $\mathbf{i} \in \mathcal{R}(w)$ (Lemma 2.1). Hence we may assume towards a contradiction that $w$ avoids $(-1,-2)$ but contains an occurrence of $(1,-3,-2)$, in positions $i<j<k$. Among all such occurrences 
of this pattern, choose one that minimizes $i+j+k$. Since 1012101 is a reduced word for the member of $B_{3}$ whose one-line form is $(1,-3,-2)$, it follows that if $(i, j, k)=(1,2,3)$, then $w$ has a reduced word that begins with 1012101, contradicting (b). In the remaining cases, it suffices to prove that there is an index $l$ with $\ell\left(s_{l} w\right)<\ell(w)$ such that the pattern $(1,-3,-2)$ also occurs in $s_{l} w$. Indeed, we may then argue by induction on length that there is a reduced word for $s_{l} w$ (and hence $w)$ that contains 1012101 as a subword, contradicting (b).

To prove the claim, note first that if a positive entry occurs in any position between $i$ and $k$ then we would have $w_{l}>0>w_{l+1}$ for some $l$ such that $i<l<k$. However in that case, $\ell\left(s_{l} w\right)<\ell(w)$ and the pattern $(1,-3,-2)$ still occurs in $s_{l} w$. Otherwise, every entry between $i$ and $k$ is negative. Since $w$ avoids $(-1,-2)$, minimality of $i+j+k$ forces $i, j, k$ to be consecutive. Hence the only remaining possibility is $i>1$.

Suppose that a positive entry occurs prior to $w_{i}$. This entry must be greater than $w_{i}$, by minimality of $i+j+k$. Hence there must be some index $l$ such that $1 \leq l<i$ and $w_{l}>w_{l+1}$. However in that case, $\ell\left(s_{l} w\right)<\ell(w)$ and the pattern $(1,-3,-2)$ still occurs in $s_{l} w$. Thus all entries prior to $w_{i}$, including $w_{1}$, must be negative. But then $\ell\left(s_{0} w\right)<\ell(w)$ and the pattern $(1,-3,-2)$ still occurs in $s_{0} w$.

$(\mathrm{c}) \Rightarrow(\mathrm{a})$. Proceeding by induction on $\ell(w)$, assume $\ell(w)>0$ and that $w$ avoids the pattern $(-1,-2)$. Let $j$ be an index such that $\ell\left(s_{j} w\right)<\ell(w)$, and let $w^{\prime}=\mathfrak{b}(w)$, as in Lemma 2.2. If $s_{j} w$ is $A$-reduced but $w$ is not, then we have $\ell\left(s_{j}^{\prime} w^{\prime}\right)=\ell\left(s_{j} w\right)=$ $\ell(w)-1>\ell\left(w^{\prime}\right)-1$, so $\ell\left(s_{j}^{\prime} w^{\prime}\right)>\ell\left(w^{\prime}\right)$. However in that case, Lemma 2.2 implies that $(1,-3,-2)$ occurs in $w$, contradicting $(\mathrm{c})$.

Otherwise, $s_{j} w$ is not $A$-reduced, so by induction $s_{j} w$ must contain the pattern $(-1,-2)$ or $(1,-3,-2)$. If the pattern $(-1,-2)$ occurs, then it must also occur in $w$ (Lemma 2.1), contradicting (c). Thus we may assume there is a subsequence $(a, b, c)$ of the one-line form of $s_{j} w$ fitting the pattern $(1,-3,-2)$; i.e., $-b>-c>a>0$. The action of $s_{j}$ cannot change the relative position of $a$ and $b$ since $a>b$ and $\ell\left(s_{j} w\right)<\ell(w)$; it also cannot change the relative position of $b$ and $c$, since otherwise the pattern $(-1,-2)$ would occur in $w$. And finally, if $j=0$ and $s_{0}$ replaces $a$ with $-a$, then the pattern $(-1,-2)$ would occur in $w$. Hence $(a, b, c)$ is also a subsequence of $w$, contradicting $(\mathrm{c})$.

Suppose that the one-line form of some $w \in A_{n}$ is $\left(w_{0}, \ldots, w_{n}\right)$, and that $m$ is the index such that $w_{m}=0$. Given any set of indices $J$ such that $\{0, m\} \subseteq J \subseteq$ $\{0,1, \ldots, m\}$, let $w^{J} \in B_{n}$ denote the result of "unbumping" the entries of $w$ in the positions indexed by $J$. More precisely, if $0=j_{0}<j_{1}<\cdots<j_{l}=m$ are the members of $J$, then define $w^{J}$ to be the member of $B_{n}$ whose one-line form is $\left(x_{1}, \ldots, x_{n}\right)$, where

$$
x_{j_{1}}=-w_{j_{0}}, \quad x_{j_{2}}=-w_{j_{1}}, \quad \ldots \quad, x_{j_{l}}=-w_{j_{l-1}},
$$

and $x_{j}=w_{j}$ for $j \notin J$.

It is not hard to see that $\mathfrak{b}(x)=w$ if and only if $x=w^{J}$ for some $J$, but it is not necessarily the case that $w^{J}$ is $A$-reduced. To characterize when this occurs, let us first introduce the notation

$$
L(w):=\left\{i: j<i \Rightarrow w_{j}>w_{i}\right\}
$$

for the set of positions where left-minima occur in the one-line form of $w \in A_{n}$. 
Theorem 2.4. If $w \in A_{n}$ and $w_{m}=0$ (i.e., $m$ is the position where 0 occurs in the one-line form of $w)$, then $x \in B_{n}$ is A-reduced and $\mathfrak{b}(x)=w$ if and only if $x=w^{J}$ with $\{0, m\} \subseteq J \subseteq L(w)$. In other words,

$$
\left\{x \in B_{n}: \mathcal{R}(x) \subseteq \mathcal{R}(w)\right\}=\left\{w^{J}:\{0, m\} \subseteq J \subseteq L(w)\right\} .
$$

Proof. Choose $J$ so that $\{0, m\} \subseteq J \subseteq L(w)$, and let $0=j_{0}<j_{1}<\cdots<j_{l}=m$ be the members of $J$. If $x=w^{J}$, it is clear from the definitions that $\mathfrak{b}(x)=w$. Furthermore, since each member of $J$ indexes a left-minimum of $w$, we have $w_{j_{0}}>\cdots>w_{j_{l}}$, and the negative entries in the one-line form of $x$ (namely, $-w_{j_{0}}, \ldots,-w_{j_{l-1}}$ ) appear in increasing order. That is, $x$ avoids the pattern $(-1,-2)$. If the pattern $(1,-3,-2)$ occurred in positions $i<j<k$ of $x$, then we would have $j, k \in J$ and $w_{i}<w_{j}$, contradicting the fact that $j$ indexes a left-minimum. Thus $x$ avoids $(1,-3,-2)$, so by Theorem 2.3 it is $A$-reduced.

Conversely, if $\mathfrak{b}(x)=w$, then $x=w^{J}$ where $J=\{0\} \cup\left\{j: x_{j}<0\right\}$. Clearly $m \in J$; in fact, $m$ is the largest member of $J$. We also claim that if $x$ is $A$-reduced, then $J \subseteq L(w)$. Otherwise, there would be some $j \in J$ such that $0<j<m$ and $j \notin L(w)$. In that case, let $k$ be the smallest member of $J$ greater than $j$ (it is clear that $k$ exists, since $m \in J)$. In the one-line form $\left(x_{1}, \ldots, x_{n}\right)$ of $x$ we must have $x_{j}<x_{k}$, since otherwise the pattern $(-1,-2)$ would appear, contrary to the assumption that $x$ is $A$-reduced. When $\mathfrak{b}$ is applied to $x,-x_{k}$ replaces the entry in position $j$. However $j \notin L(w)$, so there is an index $i<j$ such that $w_{i}<w_{j}=-x_{k}$. It is necessarily the case that $i \notin J$, since otherwise the pattern $(-1,-2)$ would have appeared in $x$. However if $i \notin J$, then the entries in positions $i, j, k$ of $x$ fit the pattern $(1,-3,-2)$. By Theorem 2.3, this contradicts the assumption that $x$ is $A$-reduced.

As a consequence of Theorem 2.4 and Proposition 1.2, we obtain the following.

Corollary 2.5. If $w \in A_{n}$ and $m$ are as above, then

$$
\mathcal{R}(w)=\bigcup_{\{0, m\} \subseteq J \subseteq L(w)} \mathcal{R}\left(w^{J}\right) .
$$

To count the $A$-reduced members of $B_{n}$, we use the following.

Lemma 2.6. We have $\sum_{w \in A_{n}} q^{\# L(w)}=q(q+1) \cdots(q+n)$.

Proof. Let $L_{n}(q)=\sum_{w \in A_{n}} q^{\# L(w)}$. We have $L_{n}(q)=(q+n) L_{n-1}(q)$, since among the $n+1$ positions where ' $n$ ' can be inserted into the one-line form of some $w \in A_{n-1}$, the number of left-minima changes only when ' $n$ ' is inserted at the beginning.

Proposition 2.7. There are $\frac{1}{4}(n+2) !+\frac{1}{2} n$ ! A-reduced members of $B_{n}$.

Proof. By Theorem 2.4, there are $2^{\# L(w)-2} A$-reduced members of $B_{n}$ corresponding to each $w \in A_{n}$ unless $\# L(w)=1$, in which case there is just one. The latter occurs when $w_{0}=0$ (i.e., when $w$ belongs to the parabolic subgroup of $A_{n}$ generated by $\left.s_{1}^{\prime}, \ldots, s_{n-1}^{\prime}\right)$. Hence the number of $A$-reduced elements is

$$
\frac{1}{4} \sum_{w \in A_{n}} 2^{\# L(w)}+\frac{1}{2} \sum_{w \in A_{n-1}} 1 .
$$

Apply Lemma 2.6. 


\section{The symmetric Functions $G_{B}$ And $G_{A}(t)$}

Let $u_{0}, u_{1}, \ldots, u_{n-1}$ denote generators for the nil Coxeter $\operatorname{ring} U$ associated with $B_{n}$. Shifting the notation of Section 1.6 slightly, let

$$
\begin{aligned}
& F_{n}^{+}(z)=\left(1+z u_{0}\right)\left(1+z u_{1}\right) \cdots\left(1+z u_{n-1}\right), \\
& F_{n}^{-}(z)=\left(1+z u_{n-1}\right) \cdots\left(1+z u_{1}\right)\left(1+z u_{0}\right),
\end{aligned}
$$

and $G_{n}(z)=F_{n}^{-}(z) F_{n}^{+}(z)$. It was first noted by Fomin and Kirillov [FK1] that $G_{n}(x)$ and $G_{n}(y)$ commute; this observation allows one to define a family of symmetric functions indexed by $w \in B_{n}$ via the method explained in Section 1.7.

To minimize notation in the following, we adopt the convention that if $u$ is a nilpotent element of a ring with unity and $x$ is a central indeterminate, then $u^{x}:=\exp (x u)$. In all cases of interest $u$ will be nilpotent of index 2 , so in fact $u^{x}=1+x u$.

Proposition 3.1. Let $u, v, a, b$ be elements of a ring with unity such that $u^{2}=$ $v^{2}=0, v$ commutes with $a$ and $b$, and $u^{x} a u^{x}$ commutes with $u^{y} b u^{y}$.

(a) If $(u v)^{2}=(v u)^{2}$, then $v^{x} u^{2 x} v^{x}$ commutes with $v^{y} u^{2 y} v^{y}$.

(b) If uvu $=v u v$, then $v^{x} u^{x} a u^{x} v^{x}$ commutes with $v^{y} u^{y} b u^{y} v^{y}$.

Proof. (a) is a straightforward computation. For (b), note first that $u^{x} v^{x}$ and $v^{x} u^{x}$ commute with $u^{y} v^{y}$ and $v^{y} u^{y}$, by the $n=2$ cases of Lemma 1.5 (with $u=u_{1}$, $\left.v=u_{2}\right)$. We therefore have

$$
\begin{aligned}
\left(v^{x} u^{x} a u^{x} v^{x}\right)\left(v^{y} u^{y} b u^{y} v^{y}\right) & =v^{x} u^{x} a\left(v^{y} u^{y}\right)\left(u^{x} v^{x}\right) b u^{y} v^{y}=v^{x} u^{x} v^{y} a u^{x+y} b v^{x} u^{y} v^{y} \\
& =\left(v^{x} u^{x}\right)\left(v^{y} u^{y}\right) u^{-y} a u^{x+y} b u^{-x}\left(u^{x} v^{x}\right)\left(u^{y} v^{y}\right) \\
& =\left(v^{y} u^{y}\right)\left(v^{x} u^{x}\right) u^{-y} a u^{x+y} b u^{-x}\left(u^{y} v^{y}\right)\left(u^{x} v^{x}\right) \\
& =\left(v^{y} u^{y}\right) v^{x} u^{-y}\left(u^{x} a u^{x}\right)\left(u^{y} b u^{y}\right) u^{-x} v^{y}\left(u^{x} v^{x}\right) \\
& =v^{y} u^{y} v^{x} u^{-y}\left(u^{y} b u^{y}\right)\left(u^{x} a u^{x}\right) u^{-x} v^{y} u^{x} v^{x} \\
& =v^{y} u^{y} v^{x} b u^{x+y} a v^{y} u^{x} v^{x}=v^{y} u^{y} b\left(v^{x} u^{x}\right)\left(u^{y} v^{y}\right) a u^{x} v^{x} \\
& =\left(v^{y} u^{y} b u^{y} v^{y}\right)\left(v^{x} u^{x} a u^{x} v^{x}\right) .
\end{aligned}
$$

Corollary 3.2 (Fomin-Kirillov). We have $G_{n}(x) G_{n}(y)=G_{n}(y) G_{n}(x)$.

Proof. Proceed by induction on $n$. The basis of the induction $(n=2)$ is a consequence of Proposition 3.1(a) and the identifications $u=u_{0}, v=u_{1}$. For $n>2$, apply Proposition 3.1(b) with $a=G_{n-2}(x), b=G_{n-2}(y), u=u_{n-2}$ and $v=u_{n-1}$.

It follows that for $w \in B_{n}$, the coefficients $G_{B}(w)$ appearing in the expansion

$$
G_{n}\left(z_{1}\right) G_{n}\left(z_{2}\right) \cdots=\sum_{w \in B_{n}} G_{B}(w)\left(z_{1}, z_{2}, \ldots\right) u_{w}
$$

are symmetric functions of $z_{1}, z_{2}, \ldots$, and by Proposition $1.4(\mathrm{~b})$ we have the expansion

$$
G_{B}(w)=\sum_{\mathbf{i} \in \mathcal{R}(w)} K_{\Lambda(\mathbf{i})}
$$


By Theorem 3.8 of [Ste1], it follows immediately that $G_{B}(w)$ is $Q$-integral, i.e., an integer linear combination of Schur $Q$-functions.

Remark 3.3. These symmetric functions have been studied previously by FominKirillov [FK1], T.-K. Lam [L], and Billey and Haiman [BH], although in some cases using the normalization $2^{-\ell_{0}(w)} G_{B}(w)$. For example, Lam and Billey-Haiman both prove that $G_{B}(w)$ is a positive integer linear combination of Schur $Q$-functions. Although it is immediate that $2^{-\ell_{0}(w)} G_{B}(w)$ is an integer linear combination of Schur $P$-functions, for combinatorial purposes, it is preferable to use the $Q$-function expansion of $G_{B}(w)$. (E.g., see the proof of Theorem 2.13 in [L].) The $Q$-integrality of $G_{B}(w)$ is also a stronger assertion than the $P$-integrality of $2^{-\ell_{0}(w)} G_{B}(w)$.

Now let $u_{0}^{\prime}, u_{1}^{\prime}, \ldots, u_{n-1}^{\prime}$ denote generators for the nil Coxeter ring $U^{\prime}$ of $A_{n}$. The only braid relation of $B_{n}$ that is not also a braid relation of $A_{n}$ is $1010 \approx 0101$. However the corresponding relation in $U$ (namely, $u_{0} u_{1} u_{0} u_{1}=u_{1} u_{0} u_{1} u_{0}$ ) is also valid in $U^{\prime}$, since $u_{0}^{\prime} u_{1}^{\prime} u_{0}^{\prime} u_{1}^{\prime}=0=u_{1}^{\prime} u_{0}^{\prime} u_{1}^{\prime} u_{0}^{\prime}$. That is, $U^{\prime}$ is a quotient of $U$. (More generally, this applies to the nil Coxeter rings of any pair $\left(W, W^{\prime}\right)$ such that $W$ dominates $W^{\prime}$.) Thus Corollary 3.2 is also valid in $U^{\prime}$, and this permits the construction of a family of symmetric functions $G_{A}(w)$ for $w \in A_{n}$ as in (3.1). But as we noted previously in Remark $1.6, G_{A}(w)$ is merely a homomorphic image of $F_{A}(w)$, and thus carries no new combinatorial information.

However, consider the following refinement.

If $t$ is any central indeterminate, the map $u_{0} \mapsto t u_{0}, u_{i} \mapsto u_{i}(1 \leq i<n)$ defines a (unique) ring endomorphism of $U$ (or rather, $U[t]$ ). Note that for $w \in B_{n}$, this map has the property that $u_{w} \mapsto t^{\ell_{0}(w)} u_{w}$. As an endomorphism, it of course preserves the commuting relationship of Corollary 3.2, but its effect on (3.1) is rather trivial-replacing $G_{B}(w)$ with $t^{\ell_{0}(w)} G_{B}(w)$. However, if we combine this with the homomorphism $U \mapsto U^{\prime}$ (i.e., $u_{0} \mapsto t u_{0}^{\prime}$, otherwise $u_{i} \mapsto u_{i}^{\prime}$ ), we obtain from Corollary 3.2 a genuinely new commuting relationship in $U^{\prime}$ with an associated family of symmetric functions indexed by $w \in A_{n}$ and depending on a parameter $t$; namely,

$$
G_{A}(w ; t):=\sum_{\mathbf{i} \in \mathcal{R}(w)} t^{\ell_{0}(\mathbf{i})} K_{\Lambda(\mathbf{i})} .
$$

Here we are committing a minor abuse of notation - using $\ell_{0}(\mathbf{i})$ to denote the number of occurrences of 0 in the reduced word $\mathbf{i}$.

Comparing (3.2) and (3.3), the following is a consequence of Corollary 2.5.

Theorem 3.4. If $w \in A_{n}$ and $w_{m}=0$ (i.e., $m$ is the position where 0 occurs in the one-line form of $w$ ), then

$$
G_{A}(w ; t)=\sum_{\{0, m\} \subseteq J \subseteq L(w)} t^{\# J-1} G_{B}\left(w^{J}\right) .
$$

Remark 3.5. It follows that for all $w \in A_{n}, G_{A}(w ; t)$ is a $\mathbf{Z}[t]$-linear combination of Schur $Q$-functions. More specifically, let $Q_{\lambda}=Q_{\lambda}\left(z_{1}, z_{2}, \ldots\right)$ denote the Schur $Q$-function indexed by the strict partition $\lambda$ (e.g., see Appendix A of [Ste1]). If $l=\ell(w)$, then $G_{A}(w ; t)$ is homogeneous of degree $l$ and it is clear from (1.1) that the coefficient of $z_{1} \cdots z_{l}$ in $K_{\Lambda}$ is $2^{l}$. On the other hand, $Q_{\lambda}$ is homogeneous of degree equal to the size of $\lambda$, and the coefficient of $z_{1} \cdots z_{l}$ in $Q_{\lambda}$ is $2^{l} g^{\lambda}$, where $g^{\lambda}$ denotes the number of shifted standard tableaux of shape $\lambda$ (e.g., [M, p. 135]). Thus for every $w \in A_{n}$ there exist polynomials $C_{w, \lambda}(t) \in \mathbf{Z}[t]$ indexed by strict 
partitions $\lambda$ of size $\ell(w)$ (and having nonnegative coefficients, by the work of Lam and Billey-Haiman) such that

$$
\sum_{\mathbf{i} \in \mathcal{R}(w)} t^{\ell_{0}(\mathbf{i})}=\sum_{\lambda} C_{w, \lambda}(t) g^{\lambda} .
$$

For example, consider $w=w_{0}$, the longest element of $A_{n}$. We have $w_{0}=(n, \ldots, 1,0)$ in one-line form, and there are $2^{n-1}$ terms in the expansion of Theorem 3.4. There is a unique term in this expansion of degree $n$ with respect to $t$ (the maximum possible), corresponding to the choice $J=L\left(w_{0}\right)=\{0,1, \ldots, n\}$. In this case $w_{0}^{J}$ is the member of $B_{n}$ whose one line-form is $(-n, \ldots,-2,-1)$. By Corollary 6.6 below (or Proposition 3.14 of [BH], or Corollary 3.5 of [L]), one knows that $G_{B}\left(w_{0}^{J}\right)=Q_{(n, \ldots, 2,1)}$. Thus we conclude that the number of reduced words for $w_{0}$ in which 0 occurs $n$ times is the number of shifted standard tableaux of shape $(n, \ldots, 2,1) .{ }^{1}$ By the shifted hook length formula for $g^{\lambda}$ (e.g., [M, p. 135]), this quantity is

$$
\left(\begin{array}{c}
n+1 \\
2
\end{array}\right) ! \cdot \prod_{i=0}^{n-1} \frac{(2 i) !}{(n+i) !} .
$$

\section{The TOP AND BOTTOM CLASSES}

For $w \in A_{n}$, recall that any $x \in B_{n}$ such that $\mathfrak{b}(x)=w$ can be obtained by unbumping the elements in some set of positions $J$ in the one-line form of $w$; i.e., $x=w^{J}$ for some $J$. In that case, $x$ has \#J-1 negative entries, and therefore $\ell_{0}(x)=\# J-1$. By Theorem 2.4, it follows that for every $w \in A_{n}$, there is a unique $x \in B_{n}$ that maximizes $\ell_{0}(x)$ among all $x^{\prime} \in B_{n}$ such that $\mathcal{R}\left(x^{\prime}\right) \subseteq \mathcal{R}(w)$. In fact, $x=w^{L(w)}$. In other words, the set of reduced words for $w$ in which 0 appears the maximum number of times is itself the set of reduced words for some $x \in B_{n}$, and this maximum number is $\# L(w)-1$. Whenever $x$ and $w$ are related in this way, we write $x=\operatorname{top}(w)$ and refer to $x$ as the top element of $w$.

Theorem 4.1. For $w \in B_{n}$, the following are equivalent.

(a) $w$ is the top element of some $w^{\prime} \in A_{n}$.

(b) 101 is not a subword of any $\mathbf{i} \in \mathcal{R}(w)$.

(c) The canonical reduced word $\left[m_{1}, n_{1}\right] \cdots\left[m_{r}, n_{r}\right]$ for $w$ satisfies $m_{1}, \ldots, m_{r} \geq$ 0 .

(d) $w$ avoids the patterns $( \pm 1,-2)$.

Proof. (a) $\Rightarrow(\mathrm{b})$. If $w$ is the top element for $w^{\prime}$, then $w$ is $A$-reduced and every $\mathbf{i} \in \mathcal{R}(w)$ is also a reduced word for $w^{\prime}$. However, if 101 occurred as a subword of $\mathbf{i}$, then $\ell_{0}(w)$ could not have been maximal since $101 \approx 010$ is a valid $A_{n}$-braid relation.

$(\mathrm{b}) \Rightarrow(\mathrm{c})$ is immediate, since 101 is a subword of $[-i, j]$ whenever $0<i \leq j$.

$(\mathrm{d}) \Rightarrow(\mathrm{a})$. If $w$ avoids the pattern $(1,-2)$, then it also avoids the pattern $(1,-3,-2)$. Hence Theorem 2.3 implies that $w$ is $A$-reduced. Now let $w^{\prime}=\mathfrak{b}(w) \in$ $A_{n}$, and let $\left(w_{1}, \ldots, w_{n}\right)$ denote the one-line form of $w$. To prove $w=\operatorname{top}\left(w^{\prime}\right)$, we must argue that $J=L\left(w^{\prime}\right)$, where $J=\{0\} \cup\left\{j: w_{j}<0\right\}$. Certainly $J \subseteq L\left(w^{\prime}\right)$, by Theorem 2.4. Now if there were some $i \in L\left(w^{\prime}\right)$ such that $i \notin J$, then there would be some $j>i$ such that $j \in J$. (Indeed, the rightmost left-minimum of $w^{\prime}$

\footnotetext{
${ }^{1}$ This is closely related to Theorem 4.5 of $[\mathrm{E}]$ — see Remark 6.3(c) below.
} 
is always a member of $J$.) If $j$ is the least such index, then when 0 is inserted into $w, w_{j}$ will be bumped and $-w_{j}$ will appear to the left of $w_{i}$ in the one-line form of $w^{\prime}$. However, $i$ indexes a left-minimum of $w^{\prime}$, so $-w_{j}>w_{i}>0$. Hence the entries in positions $i$ and $j$ of $w$ fit the pattern $(1,-2)$, contradicting (d).

To complete the proof, note that the canonical reduced words appearing in (c) are the canonical reduced words for the members of $A_{n}$, so there are exactly $(n+1)$ ! members of $B_{n}$ that satisfy (c). Therefore, having proved (d) $\Rightarrow(\mathrm{a}) \Rightarrow(\mathrm{b}) \Rightarrow(\mathrm{c})$, it suffices to prove that there are exactly $(n+1)$ ! members of $B_{n}$ that satisfy (d). For this, suppose that $w \in B_{n}$ has one-line form $\left(w_{1}, \ldots, w_{n}\right)$, and let $|w|=\left(\left|w_{1}\right|, \ldots,\left|w_{n}\right|\right)$, a permutation of $(1, \ldots, n)$. For $w$ to avoid the patterns $( \pm 1,-2)$ it is necessary and sufficient to have $j$ index a left-minimum of $|w|$ whenever $w_{j}<0$. Thus for a fixed choice of $|w|$, there are $2^{\# L(|w|)}$ ways to choose sign patterns for $w$ that avoid $( \pm 1,-2)$. Apply Lemma 2.6 .

Similarly, for each $w \in A_{n}$, Theorem 2.4 implies that there is a unique $x \in B_{n}$ that minimizes $\ell_{0}(x)$ among all $x^{\prime} \in B_{n}$ such that $\mathcal{R}\left(x^{\prime}\right) \subseteq \mathcal{R}(w)$. In other words, the set of reduced words for $w$ in which 0 appears the minimum number of times is the set of reduced words for $x$. In fact, $x$ is obtained by unbumping the 0 and the entry in position 0 from the one-line form of $w$. In this situation, we write $x=\operatorname{bot}(w)$ and refer to $x$ as the bottom element of $w$.

It is easy to show directly (or one may use Theorem 2.4 to see) that the minimum number of occurrences of 0 in any reduced word for $w \in A_{n}$ is either 0 or 1 according to whether or not 0 is the entry in position 0 of $w$. In the former case, 0 cannot appear in any reduced word for $w$, so we conclude that $x$ is a bottom element for some $w \in A_{n}$ if and only if $\ell_{0}(x) \leq 1$. Also, since $\ell_{0}(x)$ is the number of negative entries in the one-line form of $x$, this condition can also be characterized by avoidance of the patterns $(-1,-2)$ and $(-2,-1)$. We summarize these remarks with the following.

Proposition 4.2. For $w \in B_{n}$, the following are equivalent.

(a) $w$ is the bottom element of some $w^{\prime} \in A_{n}$.

(b) 010 is not a subword of any $\mathbf{i} \in \mathcal{R}(w)$.

(c) $\ell_{0}(w) \leq 1$.

(d) $w$ avoids the patterns $(-1,-2)$ and $(-2,-1)$.

Of course there are $(n+1)$ ! bottom elements, one for each member of $A_{n}$.

Those $x \in B_{n}$ that are simultaneously top and bottom elements, so that top $(w)=$ $\operatorname{bot}(w)=x$ for some $w \in A_{n}$, are distinguished by the fact that there is exactly one term in the decompositions of Corollary 2.5 and Theorem 3.4; i.e., $\mathcal{R}(x)=\mathcal{R}(w)$ and

$$
G_{A}(w ; t)=t^{\ell_{0}(x)} G_{B}(x)
$$

By Theorem 4.1 and Proposition 4.2, one sees that this class can by characterized by forbidden patterns, forbidden subwords, or by the structure of the canonical reduced word.

Define $H(n)=\sum_{i=1}^{n} 1 / i$.

Proposition 4.3. There are $n !(1+H(n))$ elements $w \in B_{n}$ such that $\mathcal{R}(w)=$ $\mathcal{R}\left(w^{\prime}\right)$ for some $w^{\prime} \in A_{n}$ (i.e., elements that are both the top and bottom of some $\left.w^{\prime}\right)$. 
Proof. Let $w \in B_{n}$ be a top-and-bottom element. By Theorem 4.1 and Proposition 4.2 , either $w$ is one of the $n$ ! elements with $\ell_{0}(w)=0$, or else $\ell_{0}(w)=1$ and $w$ avoids the pattern $(1,-2)$. In the latter case, if $-j$ is the unique negative entry in the one-line form of $w$, it is necessary and sufficient that all entries in positions to the left of $-j$ are $>j$. If $-j$ occurs in position $i$, this can be done in $\left(\begin{array}{c}n-j \\ i-1\end{array}\right)(i-1) !(n-i)$ ! ways, for a total of

$$
\sum_{i=1}^{n} \sum_{j=1}^{n}\left(\begin{array}{c}
n-j \\
i-1
\end{array}\right)(i-1) !(n-i) !=\sum_{i=1}^{n}\left(\begin{array}{c}
n \\
i
\end{array}\right)(i-1) !(n-i) !=n ! H(n) .
$$

\section{Full commutativity}

Recall that $w$ is fully commutative if $\mathcal{R}(w)$ consists of a single commutativity class, or equivalently, if $1010,212,323, \ldots$ do not occur as subwords of any $\mathbf{i} \in \mathcal{R}(w)$.

Theorem 5.1. For $w \in B_{n}$, the following are equivalent.

(a) $w$ is fully commutative.

(b) In the canonical reduced word $\left[m_{1}, n_{1}\right] \cdots\left[m_{r}, n_{r}\right]$ for $w$, we have either

(1) $m_{1}>\cdots>m_{s}>m_{s+1}=\cdots=m_{r}=0$ for some $s \leq r$, or

(2) $m_{1}>\cdots>m_{r-1}>-m_{r}>0$.

(c) $w$ avoids the pattern $(-1,-2)$ and all patterns $(a, b, c)$ such that $|a|>b>c$ or $-b>|a|>c$.

Proof. (a) $\Rightarrow$ (b). Assume that $\left[m_{1}, n_{1}\right] \cdots\left[m_{r}, n_{r}\right]$ is the canonical reduced word for some fully commutative $w \in B_{n}$. We must have $n>n_{1}>\cdots>n_{r} \geq 0$ and $\left|m_{i}\right| \leq n_{i}$, since every canonical reduced word for $B_{n}$ has this property.

For $i>0$, the word $[-1, i] 0$ is braid-equivalent to $1010[2, i]$, and for $i>j>0$ the word $[-1, i] j$ is braid-equivalent to $[-1, j-1] j(j+1) j[j+2, i]$. Hence neither word can occur as a subword of any $\mathbf{i} \in \mathcal{R}(w)$. Since subwords of this type occur in $\left[m_{i}, n_{i}\right]\left[m_{i+1}, n_{i+1}\right]$ whenever $m_{i}<0$, we must therefore have $m_{1}, \ldots, m_{r-1} \geq 0$.

If $j>k \geq i \geq 0$, then the word $[i, j] k$ is equivalent to $[i, k-1] k(k+1) k[k+2, j]$ and hence cannot occur as a subword of any $\mathbf{i} \in \mathcal{R}(w)$ unless $k=i=0$. Since subwords of this type occur in $\left[m_{i}, n_{i}\right]\left[m_{i+1}, n_{i+1}\right]$ whenever $\left|m_{i+1}\right| \geq\left|m_{i}\right|$, we must therefore have $\left|m_{i}\right|>\left|m_{i+1}\right|$ or $m_{i}=m_{i+1}=0$ for $1 \leq i<r$, and hence (b) follows.

(c) $\Rightarrow\left(\right.$ a). If $w \in B_{n}$ is not fully commutative, then there must be some $\mathbf{i} \in \mathcal{R}(w)$ containing one or more of $1010,212,323, \ldots$ as subwords. If 1010 occurs, then $w$ contains the pattern $(-1,-2)$ (Lemma 2.1), contradicting (c). For the remaining possibilities, it suffices to prove the following.

(i) If $w$ has a reduced word that begins with $i(i-1) i$ for some $i>1$, then $w$ contains one of the patterns forbidden by $(\mathrm{c})$.

(ii) If $\ell\left(s_{j} w\right)>\ell(w)$ and $w$ contains a pattern forbidden by (c), then so does $s_{j} w$.

Given the hypothesis of (i), $w$ has reduced words that begin with $i-1$ and $i$; i.e., $\ell\left(s_{i-1} w\right)<\ell(w)$ and $\ell\left(s_{i} w\right)<\ell(w)$. Thus in the one-line form $\left(w_{1}, \ldots, w_{n}\right)$ of $w$, we have $w_{i-1}>w_{i}>w_{i+1}$, a pattern that is forbidden by (c).

For (ii), suppose that $\ell\left(s_{j} w\right)>\ell(w)$ and that $(a, b, c)$ is a subsequence of $w$ such that $|a|>b>c$ or $-b>|a|>c$. If $j=0$, then $s_{j} w$ contains one of the subsequences $( \pm a, b, c)$, both of which are forbidden by (c). If $j>0$, then $s_{j} w$ will also contain the subsequence $(a, b, c)$ unless $a$ and $b$, or $b$ and $c$, occur in positions $j$ and $j+1$. 
If $b$ and $c$ occur in positions $j$ and $j+1$, then $\ell\left(s_{j} w\right)>\ell(w)$ implies $b<c$. Hence $(a, b, c)$ must satisfy $-b>|a|>c$, and $s_{j} w$ contains the subsequence $\left(a^{\prime}, b^{\prime}, c^{\prime}\right)=$ $(a, c, b)$. However this yields $\left|a^{\prime}\right|>b^{\prime}>c^{\prime}$, a pattern forbidden by (c).

If $a$ and $b$ occur in positions $j$ and $j+1$, then $\ell\left(s_{j} w\right)>\ell(w)$ implies $a<b$. Hence $(a, b, c)$ must satisfy $-a>b>c$ or $-b>a>c$, and $s_{j} w$ contains the subsequence $\left(a^{\prime}, b^{\prime}, c^{\prime}\right)=(b, a, c)$. If $-b>a>c$, then $\left|a^{\prime}\right| \geq-a^{\prime}>b^{\prime}>c^{\prime}$, and hence $\left|a^{\prime}\right|>b^{\prime}>c^{\prime}$. If $-a>b>c$, then (using also the fact that $a<b$ implies $\left.-b^{\prime}>-a^{\prime}\right)$ we must have $-b^{\prime}>\max \left(a^{\prime},-a^{\prime}\right) \geq a^{\prime}>c^{\prime}$, and hence $-b^{\prime}>\left|a^{\prime}\right|>c^{\prime}$. In either case, $\left(a^{\prime}, b^{\prime}, c^{\prime}\right)$ fits a pattern forbidden by (c).

To prove $(b) \Rightarrow(c)$, we use the following pair of lemmas.

Lemma 5.2. If the canonical reduced word $\left[m_{1}, n_{1}\right] \cdots\left[m_{r}, n_{r}\right]$ for some $w \in B_{n}$ satisfies (1), then in the one-line form of $w$ we have the following.

(a) The entries $n_{1}+1, \ldots, n_{s}+1$ occur in positions $m_{1}, \ldots, m_{s}$, respectively.

(b) The negative entries are $-\left(n_{s+1}+1\right), \ldots,-\left(n_{r}+1\right)$.

(c) The subsequence formed by the entries not specified in (a) is increasing.

Proof. Proceed by induction on $r$. If $r=1$, one can check that

$$
\begin{aligned}
\left(1,2, \ldots, m_{1}-1, n_{1}+1, m_{1}, \ldots, n_{1}, n_{1}+2, \ldots, n\right) & \left(\text { if } m_{1}>0\right), \\
\left(-\left(n_{1}+1\right), 1,2, \ldots, n_{1}, n_{1}+2, \ldots, n\right) & \left(\text { if } m_{1}=0\right),
\end{aligned}
$$

is the one-line form of $w$, and it is clear that properties (a)-(c) hold. For $r \geq 2$, let $\left(w_{1}^{\prime}, \ldots, w_{n}^{\prime}\right)$ denote the one-line form of the element $w^{\prime} \in B_{n}$ whose canonical reduced word is $\left[m_{2}, n_{2}\right] \cdots\left[m_{r}, n_{r}\right]$. Every entry $>n_{1}$ appears in its natural position in $w^{\prime}$.

If $m_{1}=0$ then $m_{2}=\cdots=m_{r}=0$. Hence by the induction hypothesis, the negative entries of $w^{\prime}$ are $-\left(n_{2}+1\right), \ldots,-\left(n_{r}+1\right)$ and we have $w_{1}^{\prime}<\cdots<w_{n}^{\prime}$. The entry $n_{1}+1$ appears in its natural position in $w^{\prime}$, so the effect of passing from $w^{\prime}$ to $w$ is to delete $n_{1}+1$ and insert $-\left(n_{1}+1\right)$ into the first position. Thus the one-line form of $w$ is increasing and the negative entries are $-\left(n_{1}+1\right), \ldots,-\left(n_{r}+1\right)$, in agreement with (a)-(c).

If $m_{1}>0$, then the one-line form of $w$ is

$$
\left(w_{1}^{\prime}, \ldots, w_{m_{1}-1}^{\prime}, n_{1}+1, w_{m_{1}}^{\prime}, \ldots, w_{n_{1}}^{\prime}, n_{1}+2, \ldots, n\right) .
$$

Since $m_{1}>m_{2}>\cdots>m_{s}>0$, it follows that $w^{\prime}$ and $w$ agree at positions $m_{2}, \ldots, m_{s}$, which by the induction hypothesis are occupied by $n_{2}+1, \ldots, n_{s}+1$. Also, we see that $w$ and $w^{\prime}$ have the same negative entries, and deletion of $n_{1}+1$ from $w$ and $w^{\prime}$ yields the same sequence, in agreement with (a)-(c).

Lemma 5.3. If the canonical reduced word $\left[m_{1}, n_{1}\right] \cdots\left[m_{r}, n_{r}\right]$ for some $w \in B_{n}$ satisfies (2), then in the one-line form of $w$ we have the following.

(a) The entries $n_{1}+1, \ldots, n_{r-1}+1$ occur in positions $m_{1}, \ldots, m_{r-1}$, respectively.

(b) The entry $-\left(n_{r}+1\right)$ occurs in the first position $>\left|m_{r}\right|$ not in $\left\{m_{1}, \ldots, m_{r-1}\right\}$.

(c) The subsequence of entries not specified in (a) and (b) is positive and increasing.

Proof. Again by induction on $r$. If $r=1$ then the one-line form of $w$ is

$$
\left(1,2, \ldots,\left|m_{1}\right|,-\left(n_{1}+1\right),\left|m_{1}\right|+1, \ldots, n_{1}, n_{1}+2, \ldots, n\right),
$$

and it is clear that properties (a)-(c) hold. 
For $r \geq 2$, let $\left(w_{1}^{\prime}, \ldots, w_{n}^{\prime}\right)$ denote the one-line form of the element $w^{\prime} \in B_{n}$ whose canonical reduced word is $\left[m_{2}, n_{2}\right] \cdots\left[m_{r}, n_{r}\right]$. Every entry $>n_{1}$ appears in its natural position in $w^{\prime}$, and the one-line form of $w$ is given by (5.1). Since $m_{1}>\cdots>m_{r-1}>0$, it follows that $w^{\prime}$ and $w$ agree at positions $m_{2}, \ldots, m_{r-1}$, which by the induction hypothesis are occupied by $n_{2}+1, \ldots, n_{r-1}+1$. Thus (a) holds. For (b), let $m$ be the position where $-\left(n_{r}+1\right)$ occurs in $w^{\prime}$. By the induction hypothesis, $m$ is the least integer $>\left|m_{r}\right|$ not in $\left\{m_{2}, \ldots, m_{r-1}\right\}$, so in particular $m \leq m_{1}$. On the other hand, in passing from $w$ to $w^{\prime}$, the position of the entry $-\left(n_{r}+1\right)$ will change only if $m \geq m_{1}$ (and hence $\left.m=m_{1}\right)$, in which case it moves to position $m_{1}+1$. Either way, the new position is the least integer $>\left|m_{r}\right|$ not in $\left\{m_{1}, \ldots, m_{r-1}\right\}$, proving (b). Finally, note that deletion of $n_{1}+1$ from $w$ and $w^{\prime}$ yields the same sequence, so (c) holds as well.

Lemmas 5.2 and 5.3 each uniquely determine the one-line form of any member of $B_{n}$ whose canonical reduced word satisfies the stated hypotheses.

To complete the proof of Theorem 5.1, let $w \in B_{n}$ be such that the canonical reduced word fits either of the two specifications in (b).

If $w$ is of the first type, then the negative entries of $w$ appear in increasing order (Lemma 5.2), so $w$ avoids $(-1,-2)$. Therefore consider an arbitrary 3 -element subsequence $(a, b, c)$ taken from the one-line form of $w$. Parts (a) and (c) of Lemma 5.2 show that $w$ can be partitioned into two increasing subsequences, so $a>b>c$ is impossible. If $-a>b>c$ were to occur with $a<0$, Lemma 5.2 shows that $-a=n_{j}+1$ for some $j>s$, and since $b>c$, either $b$ or $c$ must be $n_{i}+1$ for some $i \leq s$. However in that case, we have $i \leq s<j$ and $n_{i}>n_{j}$, so $-a<b$ or $-a<c$, a contradiction.

If $-b>|a|>c$, then $-b=n_{j}+1$ for some $j>s$ and $a>0$. (If $a<0$ then the pattern $(-1,-2)$ would appear.) Since $a$ precedes $b$ and $a>b$, we must have $a=n_{i}+1$ for some $i \leq s$, otherwise we would contradict Lemma 5.2(c). But then $i \leq s<j, n_{i}>n_{j}$ and $a>-b$, a contradiction.

If $w$ is of the second type, then $\ell_{0}(w)=1$. Hence $w$ has one negative entry, and in particular, avoids $(-1,-2)$. So consider an arbitrary 3 -element subsequence $(a, b, c)$ taken from the one-line form of $w$. By Lemma 5.3, $w$ can be partitioned into three increasing subsequences, two of which are $\left(-\left(n_{r}+1\right)\right)$ and $\left(n_{r-1}+1, \ldots, n_{1}+1\right)$. Thus $a>b>c$ can occur only if $-c=n_{r}+1$. However by Lemma 5.3(b), if $-\left(n_{r}+1\right)$ occurs in position $m$, then the entries $n_{i}+1$ that appear prior to $-\left(n_{r}+1\right)$ occur in a contiguous block from $\left|m_{r}\right|+1$ to $m-1$. Also by Lemma 5.3, the entries in positions prior to this block are smaller and in increasing order. That is, the subsequence of entries prior to $-\left(n_{r}+1\right)$ is increasing, so $a>b>c$ is impossible.

If $-a>b>c$ were to occur, then $-a=n_{r}+1$, and neither $b$ nor $c$ can be of the form $n_{i}+1$ for $i<r$ since $n_{i}+1>-a$. However $b$ and $c$ appear in decreasing order, contradicting Lemma 5.3(c). Finally, if $-b>|a|>c$, then we would have $-b=n_{r}+1$ and $a>c>0$. Hence, $a$ or $c$ must be $n_{i}+1$ for some $i<r$. However $n_{i}>n_{r}$, so $a>-b$ or $c>-b$, a contradiction.

Corollary 5.4. Every fully commutative $w \in B_{n}$ is either a top or bottom element. In particular, every fully commutative element is A-reduced.

Proof. Suppose that $w \in B_{n}$ is fully commutative. If the canonical reduced word for $w$ belongs to the first of the two types listed in Theorem 5.1(b), then $w$ is a top 
element, by Theorem 4.1. The only other possibility is $\ell_{0}(w)=1$, in which case $w$ is a bottom element by Proposition 4.2.

Remark 5.5. For "most" pairs of Coxeter groups $\left(W, W^{\prime}\right)$ such that $W$ dominates $W^{\prime}$, it is not true that the fully commutative members of $W$ are $W^{\prime}$-reduced. For example, using the most obvious labeling of the generators for the pair $\left(F_{4}, A_{4}\right)$, it is not hard to show that 4323412321 is a reduced word for some fully commutative $w \in F_{4}$. However, it is not $A_{4}$-reduced.

If we specialize to either the top or bottom classes of fully commutative elements, the forbidden patterns of Theorem 5.1 can be simplified. For example, the patterns $(a, b, c)$ such that $|a|>b>c$ are

$$
( \pm 3,2, \pm 1),( \pm 3, \pm 1,-2),( \pm 2, \pm 1,-3),( \pm 1,-2,-3) .
$$

However $w$ is a top element if and only if $w$ avoids $( \pm 1,-2)$ (Theorem 4.1 ), and the only patterns in this list that manage to avoid $( \pm 1,-2)$ belong to the first group. Note also that $(a, b)$ fits the pattern $( \pm 1,-2)$ whenever $-b>|a|>c$. Summarizing, we have

Corollary 5.6. For $w \in B_{n}$, the following are equivalent.

(a) $w$ is a fully commutative top element.

(b) The canonical reduced word $\left[m_{1}, n_{1}\right] \cdots\left[m_{r}, n_{r}\right]$ for $w$ satisfies $m_{1}>\cdots>m_{s}>m_{s+1}=\cdots=m_{r}=0$ for some $s \leq r$.

(c) $w$ avoids the patterns $( \pm 1,-2)$ and $( \pm 3,2, \pm 1)$.

Similarly, $w$ is a bottom element if and only if $\ell_{0}(w) \leq 1$ (Proposition 4.2), so the forbidden patterns for fully commutative bottom elements are $(-1,-2),(-2,-1)$, and the patterns $(a, b, c)$ of Theorem 5.1(c) with at most one negative member. Thus we have

Corollary 5.7. For $w \in B_{n}$, the following are equivalent.

(a) $w$ is a fully commutative bottom element.

(b) The canonical reduced word $\left[m_{1}, n_{1}\right] \cdots\left[m_{r}, n_{r}\right]$ for $w$ satisfies $m_{1}>\cdots>m_{r-1}>\left|m_{r}\right|$.

(c) $w$ avoids the patterns $(-1,-2),(-2,-1),(-3,2,1),(2,-3,1)$, and all patterns $(a, b, c)$ such that $a>b>c$.

Aside from a change of coordinates, the $(\mathrm{a}) \Leftrightarrow(\mathrm{c})$ parts of Corollaries 5.6 and 5.7 are implicit in the remarks of C. K. Fan in $[\mathrm{F}, \S 11]$.

If we restrict Theorem 5.1 to the subgroup of type $A_{n-1}$ generated by $s_{1}, \ldots$, $s_{n-1}$, we obtain the following. (The $(\mathrm{a}) \Leftrightarrow(\mathrm{c})$ part of this result is due to BilleyJockusch-Stanley. See Theorem 2.1 of [BJS].)

Corollary 5.8. For $w \in A_{n-1}$, the following are equivalent.

(a) $w$ is fully commutative.

(b) The canonical reduced word $\left[m_{1}, n_{1}\right] \cdots\left[m_{r}, n_{r}\right]$ for w satisfies $m_{1}>\cdots>m_{r}$.

(c) $w$ avoids the pattern $(3,2,1)$.

Let $C(n)=\frac{1}{n+1}\left(\begin{array}{c}2 n \\ n\end{array}\right)$ denote the $n$th Catalan number. Results equivalent to parts (b) and (c) of the following have also been obtained by Fan [private communication] (but stated only as a conjecture in $[\mathrm{F}]$ ).

Proposition 5.9. In $B_{n}$, there are 
(a) $(n+2) C(n)-1$ fully commutative elements.

(b) $\left(\begin{array}{c}2 n \\ n\end{array}\right)$ fully commutative top elements.

(c) $C(n+1)+C(n)-1$ fully commutative bottom elements.

(d) $C(n+1)$ fully commutative top-and-bottom elements.

Proof. By Corollary 5.6, the fully commutative top elements are encoded by pairs of integer sequences $n_{1}>\cdots>n_{r} \geq 0$ and $m_{1}>\cdots>m_{s}>0$ such that $r \geq s \geq 0, n>n_{1}$, and $n_{i}>m_{i}$. If $n_{r}=0$ then $r>s$, so we can create a new valid "code" by deleting $n_{r}$ from the first sequence. Conversely, if $n_{r}>0$, then adding $n_{r+1}=0$ to the first sequence also creates a valid code. Hence, the number of fully commutative top elements is twice the number of codes such that $n_{r}>0$. However, the codes with this property are in one-to-one correspondence with column-strict plane partitions having at most two columns and entries taken from $\{1, \ldots, n-1\}$.

Via the rule for the Schur function expansion of products of elementary symmetric functions (e.g., [M, I.(5.17)]), it follows that $\left(\begin{array}{c}n-1 \\ k\end{array}\right)^{2}$ (resp., $\left.\left(\begin{array}{c}n-1 \\ k\end{array}\right)\left(\begin{array}{c}n-1 \\ k+1\end{array}\right)\right)$ is the number of plane partitions of the desired type with a total of $2 k$ (resp., $2 k+1$ ) entries. Hence, the total number of these plane partitions is

$\sum_{k \geq 0}\left(\begin{array}{c}n-1 \\ k\end{array}\right)^{2}+\left(\begin{array}{c}n-1 \\ k\end{array}\right)\left(\begin{array}{c}n-1 \\ k+1\end{array}\right)=\sum_{k \geq 0}\left(\begin{array}{c}n-1 \\ k\end{array}\right)\left(\begin{array}{c}n \\ k+1\end{array}\right)=\left(\begin{array}{c}2 n-1 \\ n-1\end{array}\right)=\frac{1}{2}\left(\begin{array}{c}2 n \\ n\end{array}\right)$

and thus (b) follows.

By Corollary 5.8, the fully commutative members of $A_{n-1}$ are encoded by pairs of integer sequences $n>n_{1}>\cdots>n_{r}>0$ and $m_{1}>\cdots>m_{r}>0$ such that $r \geq 0$ and $m_{i}<n_{i}$. The fact that there are exactly $C(n)$ such codes can be shown in several ways: e.g., by using Schur functions to count the appropriate set of plane partitions, or by recognizing that $\left(m_{r}, n_{r}\right), \ldots,\left(m_{1}, n_{1}\right)$ can be viewed as the northto-east turning points of an increasing lattice path from $(0,0)$ to $(n, n)$ confined to the region $\{(i, j): i \leq j\}$ (a well-known interpretation of $C(n)$ ), or by appealing to the fact $C(n)$ is known to be the number of $(3,2,1)$-avoiding permutations of $n$ objects (see the discussion in [BJS, §2]).

By Corollary 5.7, the codes of this type such that $r>0$ are in one-to-one correspondence with the fully commutative bottom elements of $B_{n}$ that are not also top elements. Since there is just one code with $r=0$, it follows that there are $C(n)-1$ such elements. Hence there are $\left(\begin{array}{c}2 n \\ n\end{array}\right)+C(n)-1=(n+2) C(n)-1$ fully commutative elements, in agreement with (a).

The fully commutative bottom elements that we have not yet accounted for are those that are also top elements. However any such $w \in B_{n}$ has the property that $\mathcal{R}(w)=\mathcal{R}\left(w^{\prime}\right)$ for some fully commutative $w^{\prime} \in A_{n}$, and conversely. Hence there are $C(n+1)$ such elements (this can also be seen by examining the codes of the corresponding canonical reduced words), yielding (c) and (d).

\section{HEAPS AND HEAP EXPANSIONS}

6.1 Heaps of fully commutative elements. Suppose that $\left[m_{1}, n_{1}\right] \cdots\left[m_{r}, n_{r}\right]$ is the canonical reduced word for some fully commutative top element $w \in B_{n}$. By Corollary 5.6, we know that $n>n_{1}>\cdots>n_{r} \geq 0, m_{1}>\cdots>m_{s}>$ $m_{s+1}=\cdots=m_{r}=0$ (for some $s \leq r$ ) and $m_{i} \leq n_{i}$ for $1 \leq i \leq r$. Under these circumstances, we will say that $w$ is of shape $\lambda / \mu$, where $\lambda:=\left(n_{1}+1, \ldots, n_{r}+1\right)$ 
and $\mu:=\left(m_{1}, \ldots, m_{s}\right)$. This terminology reflects the fact that $\lambda$ and $\mu$ are a pair of strict partitions with the (shifted) diagram of $\mu$ being contained in the (shifted) diagram of $\lambda$; thus $\lambda / \mu$ may (and shall) be regarded as a shifted skew shape. Every shifted skew shape without empty rows is the shape of some fully commutative top element of $B_{n}$, provided that $n$ is sufficiently large.

More explicitly, given a strict partition $\nu_{1}>\cdots>\nu_{l}>0$, the shifted diagram of $\nu$ is defined to be

$$
D_{\nu}^{\prime}:=\left\{(i, j) \in \mathbf{Z}^{2}: 1 \leq i \leq l, i \leq j<\nu_{i}+i\right\},
$$

partially ordered so that $(i, j) \leq\left(i^{\prime}, j^{\prime}\right)$ for $i \leq i^{\prime}$ and $j \leq j^{\prime}$. Whenever $D_{\mu}^{\prime} \subseteq D_{\lambda}^{\prime}$, we write $D_{\lambda / \mu}^{\prime}$ as an abbreviation for the shifted skew diagram $D_{\lambda}^{\prime}-D_{\mu}^{\prime}$, a subposet of $D_{\lambda}^{\prime}$. We prefer to regard each shifted skew diagram as a labeled poset (in the sense of [Ste1]), with the labeling of the cell $(i, j) \in D_{\lambda / \mu}^{\prime}$ defined to be $j-i$.

For example, using matrix-style coordinates (so that poset gravity points in the northwest direction), the labeling of the cells of $\lambda / \mu=7542 / 42$ is

$$
\begin{array}{lllll} 
& & 4 & 5 & 6 \\
& 2 & 3 & 4 & \\
0 & 1 & 2 & 3 & \\
& 0 & 1 . & &
\end{array}
$$

If $w$ is a fully commutative top element of shape $\lambda / \mu$, then the canonical reduced word for $w$ is obtained by reading the labels of the diagram of $\lambda / \mu$ in (English) reading order (i.e., by rows, left-to-right, starting with the highest row).

Recall from Section 1.2 that if $w$ is fully commutative, then $\mathcal{R}(w)$ consists of the set $\mathcal{L}(P)$ of (labeled) linear extensions of a labeled poset $P$, namely, the heap of any $\mathbf{i} \in \mathcal{R}(w)$. Since all heaps belonging to a given commutativity class are isomorphic as labeled posets, we may thus refer to the heap of $w$ without ambiguity.

Proposition 6.1. If $w \in B_{n}$ is a fully commutative top element of shape $\lambda / \mu$, then the heap of $w$ is isomorphic to $D_{\lambda / \mu}^{\prime}$ (as a labeled poset).

Proof. Let $\mathbf{i}=i_{1} \cdots i_{l}$ denote the canonical reduced word for $w$ and $P$ the corresponding heap ordering of $\{1, \ldots, l\}$, as in Section 1.2 . For $1 \leq k \leq l$, define $c_{k} \in D=D_{\lambda / \mu}^{\prime}$ to be the $k$ th cell of $D$ in reading order. We claim that the map $k \mapsto c_{k}$ is a labeled poset isomorphism $P \rightarrow D$. Since the canonical reduced word is obtained by reading the labels of $D$ in (English) order, it is clear that the map is bijective and label-preserving. Now if $r<s$ is a covering relation of the heap, then $i=i_{r}$ and $j=i_{s}$ are indices of noncommuting generators of $B_{n}$; i.e., $j=i \pm 1$. However, the cells of $D$ with label $i \pm 1$ that appear later (in reading order) than the cell $c_{r}$ are all greater than $c_{r}$ in the partial order of $D$. Conversely, a cell $c \in D$ with label $i$ is covered in the partial order only by cells with labels $i \pm 1$, so these covering relations correspond to relations of the heap.

A (shifted) standard tableau of shape $\lambda / \mu$ is by definition an order-preserving bijection $T: D_{\lambda / \mu}^{\prime} \rightarrow\{1, \ldots, l\}$. The number of such tableaux is denoted $g^{\lambda / \mu}$. Since there is an obvious equivalence between standard tableaux and linear extensions of the underlying diagram poset, we obtain the following.

Corollary 6.2. If $w \in B_{n}$ is a fully commutative top element of shape $\lambda / \mu$, then we have $\# \mathcal{R}(w)=g^{\lambda / \mu}$. 
Remark 6.3. (a) If we specialize Proposition 6.1 and its corollary to the parabolic subgroup $A_{n-1}$, we obtain Proposition 2.1 and Corollary 2.1 of [BJS].

(b) In case $\mu=\varnothing$, there is a well-known hook length formula for $g^{\lambda / \mu}$ - see [M, p. 135]. The fully commutative top elements whose shapes have this property are distinguished among all members of $B_{n}$ by the fact that their one-line forms are increasing (Lemma 5.2). Furthermore, for such elements the negative entries of the one-line form are (in absolute value) the parts of $\lambda$.

(c) The special case $\mu=\varnothing$ of Corollary 6.2 is closely related to Theorem 4.5 of [E]. In this paper, Edelman identifies a set of elements $w_{\lambda} \in A_{n}$ indexed by strict partitions $\lambda$, and proves that the number of $\mathbf{i} \in \mathcal{R}(w)$ that satisfy the lattice property is $g^{\lambda}$. Although the definition of $w_{\lambda}$ is complicated, it can be shown that $x=\operatorname{top}\left(w_{\lambda}^{-1}\right) \in B_{n}$ is the fully commutative top element of shape $\lambda / \varnothing$ and $\mathcal{R}\left(x^{-1}\right)$ is the set of reduced words for $w_{\lambda}$ satisfying the lattice property.

The heaps of the fully commutative members of $B_{n}$ that are not top elements are more complicated to describe. By Corollaries 5.6 and 5.7, the canonical reduced words for such elements are of the form

$$
\mathbf{i}=\left[m_{1}, n_{1}\right] \cdots\left[m_{r-1}, n_{r-1}\right]\left[-m_{r}, n_{r}\right],
$$

where $m_{1}>\cdots>m_{r}>0$. If we delete the subword [ $\left.-m_{r}, m_{r}-1\right]$ from $\mathbf{i}$, we obtain a canonical reduced word $\mathbf{j}=\left[m_{1}, n_{1}\right] \cdots\left[m_{r}, n_{r}\right]$ for some fully commutative top element. The heap of this top element is by Proposition 6.1 a shifted skew diagram of some shape, say $\lambda / \mu$. Furthermore, this diagram has the property that the smallest label is $m=m_{r}$, and there is exactly one cell with this property. In fact, in any shifted skew diagram with no cells labeled 0 (i.e., no cells on the main diagonal) the smallest label appears only once.

Since $\mathbf{i}$ can be obtained from $\mathbf{j}$ by replacing the unique occurrence of the smallest term $m$ with the word $m \cdots 101 \cdots m$, it follows that the heap of $\mathbf{i}$ can be obtained from the heap of $\mathbf{j}$ by replacing the cell labeled $m$ with a chain of $2 m+1$ elements labeled $m, \ldots, 1,0,1, \ldots, m$.

More formally, given a labeled poset $P$ with a unique vertex $x$ having label $m>0$, define $I_{m}(P)$ to be the labeled poset obtained from $P$ by replacing $x$ with the chain

$$
x_{-m}<\cdots<x_{-1}<x_{0}<x_{1}<\cdots<x_{m} .
$$

The label of $x_{i}$ is defined to be $|i|$, and for each relation $x<y$ (resp., $x>y$ ) of $P$, we now have $x_{i}<y$ (resp., $x_{i}>y$ ) for all $|i| \leq m$.

In summary, we have the following.

Proposition 6.4. If $w \in B_{n}$ is fully commutative, with a canonical reduced word of the form (6.1), then the heap of $w$ is isomorphic to $I_{m}\left(D_{\lambda / \mu}^{\prime}\right)$ (as a labeled poset), where $\lambda=\left(n_{1}+1, \ldots, n_{r}+1\right), \mu=\left(m_{1}, \ldots, m_{r}\right)$, and $m=m_{r}$.

For example, consider the fully commutative $w \in B_{8}$ whose canonical reduced word is $[5,7][3,5][-2,4]$. The shape of $[5,7][3,5][2,4]$ is $\lambda / \mu=865 / 532$ (see Figure 1) and the heap of $w$ is obtained by replacing the cell of $\lambda / \mu$ labeled 2 with a 5 -element chain. See Figure 2. As this example plainly shows, the heap of a fully commutative member of $B_{n}$ need not be isomorphic to a shifted skew diagram, or even ranked. 


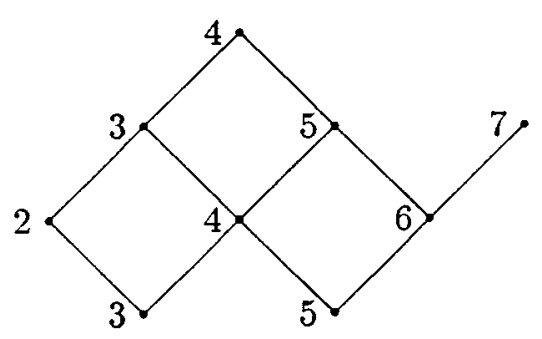

Figure 1

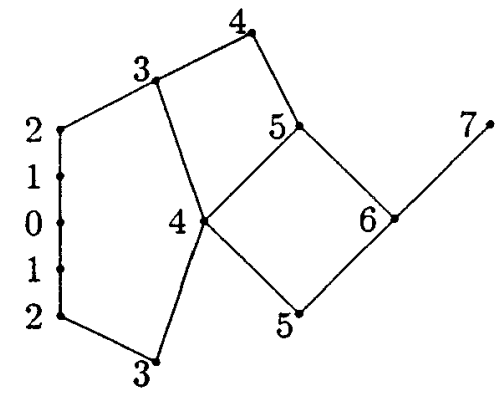

Figure 2

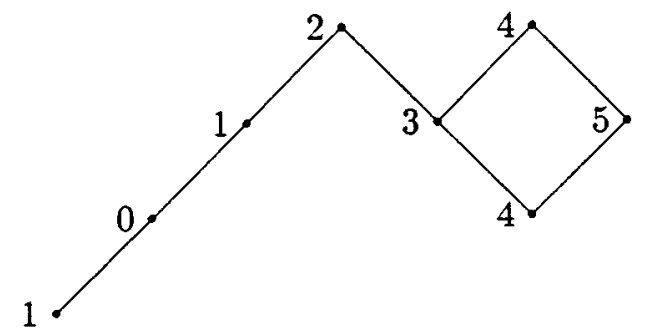

Figure 3

On the other hand, it is possible for the heaps of words of the form (6.1) to be isomorphic to shifted skew diagrams as unlabeled posets. For example, it is clear from Figure 3 that, after deleting the labels, the heap of $[4,5][3,4][-1,2]$ is isomorphic to $D_{764 / 54}^{\prime}$. Hence the number of reduced words for the corresponding element of $B_{n}$ is the number of standard shifted tableaux of shape 764/54. In general, it is not hard to show that the (unlabeled) heap of any word of the form (6.1) is isomorphic to an (unlabeled) shifted skew diagram if and only if $m_{r}+1$ occurs at most once, or equivalently, $n_{r}=m_{r}$ or $m_{r-1}>m_{r}+1$.

6.2 Heap expansions. There is a close connection between the symmetric functions $G_{B}(w)$ and the theory of enriched $P$-partitions developed in [Ste1]. To explain, let $P$ be a partial ordering of a finite set $X$, and let $\gamma: X \rightarrow\{0,1,2, \ldots\}$ be a labeling of its elements. An enriched $P$-partition is a mapping $f: P \rightarrow\{ \pm 1, \pm 2, \ldots\}$ such that the following properties hold for all $x<y$ in $P$ : (1) $f(x) \preccurlyeq f(y)$ (where $\preccurlyeq$ denotes the total ordering of Section 1.6), (2) $f(x)=f(y)>0$ implies $\gamma(x)<\gamma(y)$, and (3) $f(x)=f(y)<0$ implies $\gamma(x)>\gamma(y)$.

The primary object of study in [Ste1] is the generating function

$$
\Delta(P)\left(z_{1}, z_{2}, \ldots\right)=\sum_{f} \prod_{x \in X} z_{|f(x)|},
$$

summed over all enriched $P$-partitions $f$.

It should be noted that, in [Ste1], the labeling map of the poset $P$ is required to be injective; however the labeled posets we have in mind here (namely, heaps of reduced words) tend to have multiple uses of the same label. Nevertheless, it is easy to check that the theory of enriched $P$-partitions remains valid for non-injective labelings, provided that each element is comparable to, but does not cover, every 
other element of the same label. This is equivalent to requiring every labeled linear extensions of $P$ to be twin-free. (Hence the theory does apply to heaps of reduced words.)

Among the motivating examples of enriched $P$-partitions are the tableaux associated with Schur $Q$-functions. Indeed the Schur $Q$-function indexed by the (shifted) skew shape $\lambda / \mu$ is the generating function for enriched $D_{\lambda / \mu}^{\prime}$-partitions. That is,

$$
Q_{\lambda / \mu}=\Delta\left(D_{\lambda / \mu}^{\prime}\right) .
$$

See [Ste1, §2.4] for more details.

Proposition 6.5. If $P_{1}, \ldots, P_{k}$ are the heaps of the commutativity classes of $\mathcal{R}(w)$ for some $w \in B_{n}$, then we have

$$
G_{B}(w)=\Delta\left(P_{1}\right)+\cdots+\Delta\left(P_{k}\right) .
$$

Proof. If $P$ is the heap of any reduced word $\mathbf{i}$, then by the fundamental lemma of enriched $P$-partitions (Lemma 2.1 of [Ste1]), we have

$$
\Delta(P)=\sum_{\mathbf{j} \in \mathcal{L}(P)} K_{\Lambda(\mathbf{j})} .
$$

However $\mathcal{L}(P)$ is the commutativity class of $\mathbf{i}$ (Proposition 1.1), so the result follows from (3.2).

Results equivalent to (in some instances special cases of) the following have been independently obtained by others. For example, Lam (Corollary 3.5 of [L]) and Billey-Haiman (Proposition 3.14 of $[\mathrm{BH}]$ ) both prove the case $\mu=\varnothing$, and Fomin-Kirillov [FK1, §8] state the result without proof.

Corollary 6.6. If $w \in B_{n}$ is a fully commutative top element of shape $\lambda / \mu$, then we have $G_{B}(w)=\Delta\left(D_{\lambda / \mu}^{\prime}\right)=Q_{\lambda / \mu}$.

An interesting open problem (see $[$ Ste1, $\S 5]$ ) is the classification of labeled posets $P$ such that $\Delta(P)$ is a symmetric function. An obvious conjecture to propose is that shifted skew diagrams are the only $\Delta$-symmetric posets. However, even after accounting for the "correct" notion of isomorphism for labeled posets (namely, the weak isomorphism of [Ste1, §2.3]), the fully commutative members of $B_{n}$ (and as we shall see, also $D_{n}$ ) provide examples of $\Delta$-symmetric posets that are not of this type.

To be explicit, first note that by Proposition 6.5 we have the following.

Corollary 6.7. If $P$ is the heap of any fully commutative $w \in B_{n}$, then we have $\Delta(P)=G_{B}(w)$. In particular, $\Delta(P)$ is symmetric.

Hence by Proposition 6.4 , we obtain a $\Delta$-symmetric poset by taking any skew diagram whose smallest label is $m>0$, and replacing the (necessarily unique) cell with this label by a $(2 m+1)$-element chain. If the resulting labeled poset $P$ has more than one vertex labeled $m+1$ (as in, for example, the heap of Figure 2), then it is not isomorphic, even in the weak sense, to any shifted skew diagram.

\section{Part II. $D_{n}$}

Let $s_{\overline{1}}, s_{1}, \ldots, s_{n-1}$ denote generators for the Coxeter group $D_{n}$, arranging the indices so that $m(\overline{1}, 2)=m(1,2)=3$ and $m(i-1, i)=3$ for $2<i<n$. For any word $\mathbf{i} \in \mathcal{R}\left(D_{n}\right)$, we let $\ell_{1}(\mathbf{i})$ (resp., $\ell_{\overline{1}}(\mathbf{i})$ ) denote the number of occurrences 
of 1 (resp., $\overline{1})$, and define $\ell_{ \pm 1}(\mathbf{i})=\ell_{1}(\mathbf{i})+\ell_{\overline{1}}(\mathbf{i})$. In some circumstances, it will be necessary to have a total ordering of the indices; for these purposes, we choose $\overline{1}<1<2<\cdots<n-1$.

Interchanging $s_{1}$ and $s_{\overline{1}}$ extends to an automorphism of $D_{n}$, denoted $w \mapsto \bar{w}$. We adopt the convention that $A_{n-1}$ refers specifically to the parabolic subgroup of $D_{n}$ generated by $s_{1}, \ldots, s_{n-1}$; thus to be consistent, $\bar{A}_{n-1}$ must denote the subgroup generated by $s_{\overline{1}}, s_{2}, \ldots, s_{n-1}$.

The shortest left coset representatives for $D_{n} / D_{n-1}$ consist of

$$
\begin{aligned}
& \left\{1, s_{n-1}, s_{n-2} s_{n-1}, \ldots, s_{1} s_{2} \cdots s_{n-1}, s_{\overline{1}} s_{2} \cdots s_{n-1},\right. \\
& \left.\quad s_{1} s_{\overline{1}} s_{2} \cdots s_{n-1}, s_{2} s_{1} s_{\overline{1}} s_{2} \cdots s_{n-1}, \ldots, s_{n-1} \cdots s_{2} s_{1} s_{\overline{1}} s_{2} \cdots s_{n-1}\right\} .
\end{aligned}
$$

These coset representatives each have either one or two reduced expressions, according to whether the factor $s_{1} s_{\overline{1}}$ occurs. By consistently choosing representative reduced words in which the subword $\overline{1} 1$ does not appear, we thus obtain a canonical reduced word for every $w \in D_{n}$, following the conventions of Section 1.3.

For integers $j \geq i \geq 2$, we define $\langle i, j]$ and $\langle-i, j]$ to be the words $i \cdot(i+1) \cdots j$ and $i \cdot(i-1) \cdots 21 \overline{1} 2 \cdots j$ (respectively), and for $j \geq 1$ we define

$$
\langle 1, j]=12 \cdots j, \quad\langle-1, j]=\overline{1} 2 \cdots j, \quad\langle 0, j]=1 \overline{1} 2 \cdots j .
$$

In particular, $\langle-1,1]=\overline{1}$ and $\langle 0,1]=1 \overline{1}$. In these terms, the canonical reduced words for the members of $D_{n}$ are the expressions

$$
\left\langle m_{1}, n_{1}\right] \cdot\left\langle m_{2}, n_{2}\right] \cdots\left\langle m_{r}, n_{r}\right],
$$

where $n>n_{1}>\cdots>n_{r} \geq 1$ and $\left|m_{i}\right| \leq n_{i}$.

With $\varepsilon_{1}, \ldots, \varepsilon_{n}$ as the standard orthonormal basis of $\mathbf{R}^{n}$, we take $\varepsilon_{i+1}-\varepsilon_{i}$ (resp., $\varepsilon_{1}+\varepsilon_{2}$ ) as the simple root corresponding to $s_{i}$ for $i \geq 1$ (resp., $i=\overline{1}$ ). In these terms, the vector $\delta=\varepsilon_{1}+2 \varepsilon_{2}+\cdots+n \varepsilon_{n}=(1,2, \ldots, n)$ belongs to the interior of the fundamental chamber defined by this choice of simple roots, and its orbit consists of all signed permutations of $(1,2, \ldots, n)$ with an even number of negative entries. These constitute the one-line forms of the members of $D_{n}$.

\section{The $A$-Stable members of $D_{n}$}

The map $s_{\overline{1}} \mapsto s_{1}, s_{i} \mapsto s_{i}(i \geq 1)$ extends to a group homomorphism $D_{n} \rightarrow$ $A_{n-1}$, denoted $w \mapsto|w|$. In terms of one-line forms, the effect of this homomorphism is the same as taking the absolute values of the coordinates; i.e., $\left(w_{1}, \ldots, w_{n}\right) \mapsto$ $\left(\left|w_{1}\right|, \ldots,\left|w_{n}\right|\right)$.

If the length of $w \in D_{n}$ is the same as the length of $|w| \in A_{n-1}$, we will say that $w$ is $A$-stable. As we shall see, the $A$-stable members of $D_{n}$ are closely related to the $A$-reduced members of $B_{n-1}$.

Theorem 7.1. For $w \in D_{n}$, the following are equivalent.

(a) $w$ is A-stable.

(b) $1 \overline{1}$ is not a subword of any $\mathbf{i} \in \mathcal{R}(w)$.

(c) $1 \overline{1}$ does not occur in the canonical reduced word for $w$.

(d) $w$ avoids the patterns $( \pm 1,-2)$.

Proof. Since $\left|s_{1} s_{\overline{1}}\right|=1$, it is clear that (a) $\Rightarrow(\mathrm{b})$. Also, (b) $\Rightarrow(\mathrm{c})$ is immediate.

$(\mathrm{c}) \Rightarrow(\mathrm{d})$. Proceed by induction on $n$. If $n=2$, the possibilities for $w$ are $1, s_{1}$, and $s_{\overline{1}}$, for which the corresponding one-line forms are $(1,2),(2,1)$, and $(-2,-1)$. Otherwise, if $n>2$, consider the canonical factorization $x_{n} \cdots x_{2}$ of $w$. By the 
induction hypothesis, $w^{\prime}=x_{n-1} \cdots x_{2} \in D_{n-1}$ has a one-line form $\left(w_{1}^{\prime}, \ldots, w_{n-1}^{\prime}\right)$ that avoids the patterns $( \pm 1,-2)$. In passing from $w^{\prime}$ to $w=x_{n} w^{\prime}$, the entry $\pm n$ is inserted into some position of the one-line form depending on $x_{n}$. Since $n$ cannot participate in an occurrence of either of the patterns $( \pm 1,-2)$, suppose that $-n$ is the inserted entry. This can happen only if $\overline{1}$ occurs in the canonical reduced word for $x_{n}$. However, the only coset representative for $D_{n} / D_{n-1}$ whose canonical reduced word contains $\overline{1}$ but not $1 \overline{1}$ is $x_{n}=s_{\overline{1}} s_{2} \cdots s_{n-1}$. In that case, the oneline form of $w$ is $\left(-n,-w_{1}^{\prime}, w_{2}^{\prime}, \ldots, w_{n-1}^{\prime}\right)$ and there is no way for $-n$ (or $\left.-w_{1}^{\prime}\right)$ to participate in an occurrence of the patterns $( \pm 1,-2)$.

(d) $\Rightarrow(\mathrm{a})$. If $w$ contains one of the patterns $( \pm 1,-2)$ and $\ell\left(s_{j} w\right)>\ell(w)$, then we claim that $s_{j} w$ also contains one of these patterns. To see this, suppose that $(a, b)$ is a subsequence of the one-line form of $w$ that fits $( \pm 1,-2)$; i.e., $-b>|a|$. If $j \geq 1$ then $(a, b)$ will also be a subsequence of $s_{j} w$ unless $s_{j}$ interchanges $a$ and $b$. However since $\ell\left(s_{j} w\right)>\ell(w)$, this would require $a<b$, contrary to the fact that $-b>|a|$. In the remaining case, namely $j=\overline{1}$, we cannot have $a$ and $b$ in the first two positions of $w$, since otherwise $\ell\left(s_{j} w\right)>\ell(w)$ would require that $a+b>0$. Hence either $(a, b)$ or $(-a, b)$ occurs as a subsequence of $s_{j} w$, both of which fit $( \pm 1,-2)$.

Given the claim, it suffices to show that if $w$ is $A$-stable but $s_{j} w$ is not, then $s_{j} w$ contains one of the patterns $( \pm 1,-2)$. For this, note first that $\ell\left(s_{j} w\right)>\ell(w)$ (otherwise $s_{j} w$ would be $A$-stable) and $\ell\left(\left|s_{j} w\right|\right)<\ell\left(s_{j} w\right)=\ell(w)+1=\ell(|w|)+1$, so $\ell\left(\left|s_{j} w\right|\right)<\ell(|w|)$. If $j \geq 1$, let $a$ and $b$ denote the entries of the one-line form of $w$ in positions $j$ and $j+1$. Since $\ell\left(s_{j} w\right)>\ell(w)$, we have $a<b$, and since $\ell\left(\left|s_{j} w\right|\right)<\ell(|w|)$, we have $|a|>|b|$. Hence $-a>|b|$ and the subsequence $(b, a)$ of $s_{j} w$ fits one of the patterns $( \pm 1,-2)$. Otherwise, if $j=\overline{1}$, let $a$ and $b$ denote the entries in positions 1 and 2 of the one-line form of $w$. We have $a+b>0$ since $\ell\left(s_{j} w\right)>\ell(w)$, and $|a|>|b|$ since $\ell\left(\left|s_{j} w\right|\right)<\ell(|w|)$. Therefore $a>|b|$ and the subsequence $(-b,-a)$ of $s_{j} w$ fits one of the patterns $( \pm 1,-2)$.

For any even $J \subseteq\{1, \ldots, n\}$, let $t(J) \in D_{n}$ denote the member of $D_{n}$ whose action on $\mathbf{R}^{n}$ is to change the sign of the coordinates indexed by $J$. The elements $t(J)$ form the kernel of the homomorphism $w \mapsto|w|$.

Corollary 7.2. If $w \in A_{n-1}$, then $w^{\prime} \in D_{n}$ is A-stable and $\left|w^{\prime}\right|=w$ if and only if $w^{\prime}=t(J) w$ for some even $J \subseteq L(w)$.

Proof. By the criterion of Theorem 7.1(d), $w^{\prime} \in D_{n}$ is $A$-stable if and only if the positions where negative entries occur are left-minima of $\left|w^{\prime}\right|$.

Corollary 7.3. There are $\frac{1}{2}(n+1)$ ! A-stable members of $D_{n}$.

Proof. By Corollary 7.2, there are $2^{\# L(w)-1} A$-stable members of $D_{n}$ corresponding to each $w \in A_{n-1}$. Apply Lemma 2.6. (Alternatively, there are $n+1$ coset representatives of $D_{n} / D_{n-1}$ whose canonical reduced words do not contain $1 \overline{1}$, so the result follows by induction and the criterion of Theorem 7.1(c).)

Define a map $\sigma:\{\overline{1}, 1, \ldots, n-1\} \rightarrow\{0,1, \ldots, n-2\}$ by setting $\sigma(i)=i-1$ for $1<i<n$ and $\sigma(1)=\sigma(\overline{1})=0$. Extending $\sigma$ to the corresponding free monoid, we will write $\sigma(\mathbf{i})$ for $\sigma\left(i_{1}\right) \cdots \sigma\left(i_{l}\right)$ whenever $\mathbf{i}=i_{1} \cdots i_{l}$. Note that if $w \in D_{n}$ is $A$-stable, then any $\mathbf{i} \in \mathcal{R}(w)$ remains reduced under the identification $1=\overline{1}$. Therefore $\sigma(\mathbf{i})$, regarded as a word formed out of labels for the generators of $B_{n-1}$, is $A$-reduced. In particular, $\sigma(\mathbf{i}) \in \mathcal{R}\left(B_{n-1}\right)$. 
If $\mathbf{j} \in \mathcal{R}\left(B_{n-1}\right)$ is obtained from $\sigma(\mathbf{i})$ by the application of a single $B_{n-1}$-braid relation, then $\mathbf{j}=\sigma\left(\mathbf{i}^{\prime}\right)$ for some word $\mathbf{i}^{\prime}$ that is $D_{n}$-braid equivalent to $\mathbf{i}$, except for cases involving the relation $1010 \approx 0101$. In such cases, $\mathbf{i}$ must contain one of the subwords $212 \overline{1}, 2 \overline{1} 21,12 \overline{1} 2$, or $\overline{1} 212$. However, none of these subwords can occur if $w$ is $A$-stable. (For example, if $212 \overline{1}$ occurs, then the relation $212 \overline{1} \approx 121 \overline{1}$ shows that $1 \overline{1}$ would appear in some reduced word for $w$.) Therefore if $w$ is $A$-stable, then

$$
\sigma \mathcal{R}(w)=\bigcup_{x \in X(w)} \mathcal{R}_{B}(x)
$$

for some $X(w) \subseteq B_{n-1}$. (We use the notation $\mathcal{R}_{B}(x)$ here, rather than $\mathcal{R}(x)$, to emphasize that $x \in B_{n-1}$.) Although it is not clear a priori, we will see that $\sigma$ is injective on $\mathcal{R}(w)$.

In order to describe the set $X(w)$ appearing in (7.1), let us define

$$
N(w)=\{1\} \cup\left\{j: w_{j}<0 \text { or }\left|w_{j}\right|=1\right\},
$$

where $\left(w_{1}, \ldots, w_{n}\right)$ denotes the one-line form of some $w \in D_{n}$. Also, for any set of positions $J=\left\{j_{1}<\cdots<j_{m}\right\}$, we define

$$
\begin{aligned}
& \xi_{+}(w, J)=\#\left\{1 \leq k<m: \quad \xi_{1} \cdots \xi_{k}=+1\right\}, \\
& \xi_{-}(w, J)=\#\left\{1 \leq k<m: \quad \xi_{1} \cdots \xi_{k}=-1\right\},
\end{aligned}
$$

where $\xi_{k}$ denotes the sign of the $j_{k}$ th entry of $w$.

Recall from Section 2 that $\mathfrak{b}: B_{n-1} \rightarrow A_{n-1}$ denotes the map in which 0 is inserted into the one-line form of $x \in B_{n-1}$ from the right, and then successive negative entries are bumped. In the present context, the one-line forms for $w \in$ $A_{n-1}$ are permutations of $(1, \ldots, n)$. Thus to produce the correct one-line form of $\mathfrak{b}(x)$, we must now supplement the procedure of Section 2 by adding $(1, \ldots, 1)$ to the result. For example, if $x \in B_{5}$ has one-line form $(3,-4,5,-2,1)$, then $\mathfrak{b}(x) \in A_{5}$ (now) has one-line form $(5,4,3,6,1,2)$.

Let us also recall from Section 2 the description of the $\mathfrak{b}$-preimages of $w \in A_{n-1}$. Taking into account the shift of entries, if $k$ is the position where 1 occurs in $w$, the preimages of $w$ are the elements $w^{J} \in B_{n-1}$ obtained by unbumping the entries of $w$ at the positions indexed by $J$, for all $J$ such that $\{1, k\} \subseteq J \subseteq\{1, \ldots, k\}$.

Theorem 7.4. If $w \in D_{n}$ is A-stable, then we have the following.

(a) The restriction of $\sigma$ to $\mathcal{R}(w)$ is injective.

(b) $\sigma \mathcal{R}(w)=\bigcup_{N(w) \subseteq K \subseteq L(|w|)} \mathcal{R}_{B}\left(|w|^{K}\right)$.

(c) If $N(w) \subseteq K \subseteq L(|w|)$, then for every $\mathbf{i} \in \mathcal{R}(w)$ such that $\sigma(\mathbf{i}) \in \mathcal{R}_{B}\left(|w|^{K}\right)$, we have $\ell_{1}(\mathbf{i})=\xi_{+}(w, K)$ and $\ell_{\overline{1}}(\mathbf{i})=\xi_{-}(w, K)$.

For example, if $w=(6,7,-4,2,3,-1,5) \in D_{7}$ then $w$ is $A$-stable, $N(w)=$ $\{1,3,6\}, L(|w|)=\{1,3,4,6\}$, and there are two elements $|w|^{K} \in B_{6}$ that appear in the decomposition of part (b), namely, $(6,-5,1,2,-3,4)$ and $(6,-5,-3,2,-1,4)$.

Corollary 7.5. If $w \in D_{n}$ is A-stable, then $\# \mathcal{R}(w)=\sum_{N(w) \subseteq K \subseteq L(|w|)} \# \mathcal{R}_{B}\left(|w|^{K}\right)$.

For example, if $w$ has one-line form $(-n, \ldots,-2, \pm 1)$ (the sign of the last entry being determined by parity considerations), then $w$ is $A$-stable and $N(w)=$ $L(|w|)=\{1, \ldots, n\}$. Hence there is exactly one term in the expansion of Corollary 7.5, corresponding to the element $x \in B_{n-1}$ whose one-line form is 
$(-(n-1), \ldots,-1)$. As noted in Section 3, the number of reduced words for $x$ (and therefore also $w)$ is the number of shifted standard tableaux of shape $(n-1, \ldots, 1)$.

If $x \in B_{n-1}$ has one-line form $\left(x_{1}, \ldots, x_{n-1}\right)$, set $M(x):=\{1\} \cup\left\{j+1: x_{j}<0\right\}$. Our proof of Theorem 7.4 relies on the following.

Lemma 7.6. For $x \in B_{n-1}$ and $w \in D_{n}$, the following are equivalent.

(a) $w$ is A-stable and $\mathcal{R}(x) \subseteq \sigma \mathcal{R}(w)$.

(b) $x$ is A-reduced and $w=t(J) \mathfrak{b}(x)$ for some even $J \subseteq M(x)$.

(c) $x=|w|^{K}$ for some $K$ such that $N(w) \subseteq K \subseteq L(|w|)$.

Furthermore, if $w$ and $x$ are related as in (c), then for some $\mathbf{i} \in \mathcal{R}(w)$ such that $\sigma(\mathbf{i}) \in \mathcal{R}(x)$, we have $\ell_{1}(\mathbf{i})=\xi_{+}(w, K)$ and $\ell_{1}(\mathbf{i})=\xi_{-}(w, K)$.

Proof. (b) $\Rightarrow(\mathrm{c})$. If $w=t(J) \mathfrak{b}(x)$ for some even $J \subseteq M(x)$, then $|w|=\mathfrak{b}(x)$. By Theorem 2.4, it follows that if $x$ is $A$-reduced, then we must have $x=|w|^{K}$ for some $K$ satisfying $\{1, k\} \subseteq K \subseteq L(|w|)$, where $k$ is the position where 1 occurs in $|w|$. Thus to satisfy (c), it remains only to check that $N(w) \subseteq K$. Since $J$ is the set of indices where negative entries occur in $w$, it suffices to show that $J \subseteq K$. However $J \subseteq M(x)$, so $j \in J$ implies $j=1$ or $x_{j-1}<0$. In either case, $j$ indexes a position that must be unbumped in order to obtain $x$ from $|w|$; i.e., $j \in K$.

(c) $\Rightarrow\left(\right.$ a). Suppose that $x=|w|^{K}$ for some $K$ such that $N(w) \subseteq K \subseteq L(|w|)$. Under these circumstances, we must have $K=M(x)$ and $|w|=\mathfrak{b}(x)$. We prove by induction on $\ell(x)$ that there exists some $\mathbf{i} \in \mathcal{R}(w)$ such that $\sigma(\mathbf{i}) \in \mathcal{R}(x), \ell_{1}(\mathbf{i})=$ $\xi_{+}(w, K)$, and $\ell_{\overline{1}}(\mathbf{i})=\xi_{-}(w, K)$. For the base of the induction, suppose $\ell_{0}(x)=0$. In that case, $x$ has no negative entries, so $K=M(x)=\{1\}$ and 1 is the first entry in the one-line form of $|w|=\mathfrak{b}(x)$. Hence $w$ belongs to the subgroup generated by $s_{2}, \ldots, s_{n-1}$ and $\mathcal{R}(x)=\sigma \mathcal{R}(w)$. We also have $\ell_{1}(\mathbf{i})=\ell_{\overline{1}}(\mathbf{i})=\xi_{ \pm}(w, K)=0$ for every $\mathbf{i} \in \mathcal{R}(w)$.

For the remainder of the proof, let $\left(w_{1}, \ldots, w_{n}\right)$ and $\left(x_{1}, \ldots, x_{n-1}\right)$ denote the respective one-line forms of $w$ and $x$. Also, to distinguish the generators of $D_{n}$ from those $B_{n-1}$, we use $s_{0}^{*}, \ldots, s_{n-2}^{*}$ to denote the latter.

In the case $\ell_{0}(x)>0, x$ has at least one negative entry; assume that the leftmost one is $x_{j}=-a$. Let $-b \leq 0$ be the entry that bumps $-a$ when 0 is inserted from the right. In $|w|=\mathfrak{b}(x)$, we therefore have $\left|w_{1}\right|=a+1$ and $\left|w_{j+1}\right|=b+1$. Since $j+1 \in M(x)=K \subseteq L(|w|), b+1$ must be a left-minimum of $|w|$.

Case 1: $j \geq 2$. Since $N(w) \subseteq K$ and $j+1$ is the smallest member of $M(x)=K$ greater than 1 , we have $w_{j}>0$. Therefore $w_{j}>w_{j+1}$ and $\ell\left(s_{j} w\right)<\ell(w)$, since $\left|w_{j+1}\right|=b+1$ is a left-minimum of $|w|$. We also have $x_{j-1}>x_{j}$ and $\ell\left(s_{j-1}^{*} x\right)<\ell(x)$, since every entry of $x$ prior to $j$ is positive. Using $s_{j} w$ and $s_{j-1}^{*} x$ in place of $w$ and $x$, the hypotheses of (c) are satisfied (the only effects on the values of $N(w), K$, and $L(|w|)$ are that the occurrences of $j+1$ are replaced with $j)$, so by the induction hypothesis we can find some $\mathbf{i}^{\prime} \in \mathcal{R}\left(s_{j} w\right)$ such that $\sigma\left(\mathbf{i}^{\prime}\right) \in \mathcal{R}\left(s_{j-1}^{*} x\right)$, with the values of $\ell_{1}\left(\mathbf{i}^{\prime}\right), \ell_{\overline{1}}\left(\mathbf{i}^{\prime}\right)$ as desired. By inserting $j$ at the beginning of $\mathbf{i}^{\prime}$, we obtain a word $\mathbf{i} \in \mathcal{R}(w)$ such that $\sigma(\mathbf{i}) \in \mathcal{R}(x)$. For this word, we have $\ell_{1}(\mathbf{i})=\xi_{+}(w, K)$ and $\ell_{\overline{1}}(\mathbf{i})=\xi_{-}(w, K)$, since the values of $\ell_{1}(\cdot), \ell_{\overline{1}}(\cdot)$ and $\xi_{ \pm}(\cdot)$ do not change.

Case 2: $j=1$. In this case, $(a+1, b+1)$ are the first two entries of $|w|$, and $a>b$ since $b+1$ is a left-minimum. Hence $\ell\left(w^{\prime}\right)<\ell(w)$, where $w^{\prime}=s_{1} w$ (if $w_{1}=a+1$ ) or $w^{\prime}=s_{\overline{1}} w$ (if $w_{1}=-(a+1)$ ). We also have $\ell\left(s_{0}^{*} x\right)<\ell(x)$, since $x_{1}=-a<0$. If we replace $w$ with $w^{\prime}$ and $x$ with $s_{0}^{*} x$, the hypotheses of (c) are still satisfied - the effects on $N(w), K$, and $L(|w|)$ are such that $j+1=2$ is deleted from $K, L(|w|)$, 
and (if it occurs there) $N(w)$. Hence by the induction hypothesis we can find some $\mathbf{i}^{\prime} \in \mathcal{R}\left(w^{\prime}\right)$ such that $\sigma\left(\mathbf{i}^{\prime}\right) \in \mathcal{R}\left(s_{0}^{*} x\right), \ell_{1}\left(\mathbf{i}^{\prime}\right)=\xi_{+}\left(w^{\prime}, K^{\prime}\right)$, and $\ell_{\overline{1}}\left(\mathbf{i}^{\prime}\right)=\xi_{-}\left(w^{\prime}, K^{\prime}\right)$, where $K^{\prime}=K-\{2\}$. By inserting 1 or $\overline{1}$ at the beginning of $\mathbf{i}^{\prime}$ according to the sign of $w_{1}$, we thus obtain a reduced word $\mathbf{i} \in \mathcal{R}(w)$ such that $\sigma(\mathbf{i}) \in \mathcal{R}(x)$. Furthermore, if $\xi_{1}, \ldots, \xi_{m}$ (resp., $\xi_{1}^{\prime}, \ldots, \xi_{m-1}^{\prime}$ ) denote the signs used to compute $\xi_{ \pm}(w, K)$ (resp., $\left.\xi_{ \pm}\left(w^{\prime}, K^{\prime}\right)\right)$ in $(7.2)$, then $\xi_{1}$ is the sign of $w_{1}$, and

$$
\left(\xi_{2}, \ldots, \xi_{m}\right)=\left(\xi_{1} \xi_{1}^{\prime}, \xi_{2}^{\prime}, \ldots, \xi_{m-1}^{\prime}\right) .
$$

It follows that $\xi_{1} \cdots \xi_{k+1}=\xi_{1}^{\prime} \cdots \xi_{k}^{\prime}$ for $k \geq 1$, so we have $\xi_{+}(w, K)-\xi_{+}\left(w^{\prime}, K^{\prime}\right)=1$ and $\xi_{-}(w, K)-\xi_{-}\left(w^{\prime}, K^{\prime}\right)=0$ or vice-versa, according to whether the first term of $\mathbf{i}$ is 1 or $\overline{1}$. Hence $\ell_{1}(\mathbf{i})=\xi_{+}(w, K)$ and $\ell_{\overline{1}}(\mathbf{i})=\xi_{-}(w, K)$, so the induction is complete.

Since one of the hypotheses of (c) is $N(w) \subseteq L(|w|)$, it follows that $w$ is $A$-stable, by Corollary 7.2. Having already shown $\mathcal{R}(x) \cap \sigma \mathcal{R}(w)$ is nonempty, it now follows that $\mathcal{R}(x) \subseteq \sigma \mathcal{R}(w)$, by $(7.1)$.

(a) $\Rightarrow$ (b). Given that $w$ is $A$-stable, every $x \in B_{n-1}$ such that $\mathcal{R}(x) \subseteq \sigma \mathcal{R}(w)$ is $A$-reduced. Thus the implication (a) $\Rightarrow(\mathrm{b})$ is trivial if $x$ is not $A$-reduced. Otherwise, there are exactly $2^{\# M(x)-1}=2^{\ell_{0}(x)}$ distinct elements $w \in D_{n}$ that satisfy (b). Having proved $(\mathrm{b}) \Rightarrow(\mathrm{c}) \Rightarrow(\mathrm{a})$, it follows that each of these elements also satisfy (a). On the other hand, for any $\mathbf{j} \in \mathcal{R}(x)$, there are only $2^{\ell_{0}(x)}$ possible words $\mathbf{i}$ such that $\sigma(\mathbf{i})=\mathbf{j}$. Since we have already identified $2^{\ell_{0}(x)}$ distinct members of $D_{n}$ that satisfy (a), this can only be reconciled if these are the only members of $D_{n}$ that satisfy (a).

Proof of Theorem 7.4. For (a), suppose that $\sigma(\mathbf{i})=\sigma\left(\mathbf{i}^{\prime}\right)=\mathbf{j}$ for some pair $\mathbf{i}, \mathbf{i}^{\prime} \in$ $\mathcal{R}(w)$. It follows that $\mathbf{j} \in \mathcal{R}(x)$ for some $A$-reduced $x \in B_{n-1}$. However, by the equivalence of parts (a) and (b) of Lemma 7.6, there are $2^{\ell_{0}(x)}$ distinct $A$-stable $w^{\prime} \in D_{n}$ such that $\mathbf{j} \in \sigma \mathcal{R}\left(w^{\prime}\right)$. Since there are only $2^{\ell_{0}(x)}$ words $\mathbf{i}^{\prime \prime}$ such that $\sigma\left(\mathbf{i}^{\prime \prime}\right)=\mathbf{j}$, it follows that they must be reduced words for distinct members of $D_{n}$. Hence $\mathbf{i}=\mathbf{i}^{\prime}$.

Part (b) is a corollary of (7.1) and the equivalence of parts (a) and (c) of Lemma 7.6.

For (c), we already know by Lemma 7.6 that there exists at least one $\mathbf{i} \in \mathcal{R}(w)$ such that $\sigma(\mathbf{i}) \in \mathcal{R}\left(|w|^{K}\right)$ for which $\ell_{1}(\mathbf{i})=\xi_{+}(w, K)$ and $\ell_{\overline{1}}(\mathbf{i})=\xi_{-}(w, K)$. Given another $\mathbf{i}^{\prime} \in \mathcal{R}(w)$ such that $\sigma\left(\mathbf{i}^{\prime}\right) \in \mathcal{R}(x), \sigma\left(\mathbf{i}^{\prime}\right)$ can be transformed into $\sigma(\mathbf{i})$ by means of a series of $B_{n-1}$-braid relations. Furthermore, the relation $1010 \approx 0101$ can never arise, since otherwise $w$ would not be $A$-stable. Hence the only relations involved are $\sigma$-images of $D_{n}$-braid relations that preserve the number of occurrences of both 1 and $\overline{1}$. It follows that there must exist $\mathbf{i}^{\prime \prime} \in \mathcal{R}(w)$ such that $\ell_{1}\left(\mathbf{i}^{\prime}\right)=\ell_{1}\left(\mathbf{i}^{\prime \prime}\right)$, $\ell_{\overline{1}}\left(\mathbf{i}^{\prime}\right)=\ell_{\overline{1}}\left(\mathbf{i}^{\prime \prime}\right)$, and $\sigma\left(\mathbf{i}^{\prime \prime}\right)=\sigma(\mathbf{i})$. However $\sigma$ is injective on $\mathcal{R}(w)$, so $\mathbf{i}=\mathbf{i}^{\prime \prime}$.

Remark 7.7. (a) If $w \in D_{n}$ is $A$-stable, Theorem 7.4 implies that the maximum value of $\ell_{ \pm 1}(\mathbf{i})$ for $\mathbf{i} \in \mathcal{R}(w)$ is $\# L(|w|)-1$, and the set of reduced words with this property is in one-to-one correspondence (via $\sigma$ ) with the set of reduced words for some $x \in B_{n-1}$ (namely, $\left.x=\operatorname{top}(|w|)\right)$.

(b) Similarly, the minimum value of $\ell_{ \pm 1}(\mathbf{i})$ for $\mathbf{i} \in \mathcal{R}(w)$ is $\# N(w)-1$, and the set of reduced words with this property is in one-to-one correspondence (via $\sigma$ ) with the set of reduced words for some $x \in B_{n-1}$. For a fixed choice of $|w|$, we can select an $A$-stable preimage $w$ so that $N(w)$ takes on any value in the interval 
$\{1, k\} \subseteq N \subseteq L(|w|)$, where $k$ denotes the position where 1 occurs in $|w|$. Thus every $A$-reduced $x \in B_{n-1}$ occurs as the $\ell_{ \pm 1}$-minimizer of some $A$-stable $w \in D_{n}$.

\section{The symmetric Functions $G_{D}$ And $G_{D}(s, t)$}

Let $u_{\overline{1}}, u_{1}, \ldots, u_{n-1}$ denote generators for the nil Coxeter ring of $D_{n}$, and define

$$
\begin{aligned}
& \bar{G}_{n}(z ; s, t) \\
& \quad=\left(1+z u_{n-1}\right) \cdots\left(1+z u_{2}\right)\left(1+2 s z u_{1}\right)\left(1+2 t z u_{\overline{1}}\right)\left(1+z u_{2}\right) \cdots\left(1+z u_{n-1}\right),
\end{aligned}
$$

where $z, s, t$ are central indeterminates. For each $w \in D_{n}$, we define $G_{D}(w ; s, t)$ to be the quasi-symmetric function appearing as the coefficient of $u_{w}$ in the expansion

$$
\bar{G}_{n}\left(z_{1} ; s, t\right) \bar{G}_{n}\left(z_{2} ; s, t\right) \cdots=\sum_{w \in D_{n}} G_{D}(w ; s, t)\left(z_{1}, z_{2}, \ldots\right) u_{w}
$$

Considering the relation

$$
\left(1+2 s z u_{1}\right)\left(1+2 t z u_{\overline{1}}\right)=\left(1+s u_{1}\right)\left(1+t u_{\overline{1}}\right)\left(1+t u_{\overline{1}}\right)\left(1+s u_{1}\right),
$$

one sees that $\bar{G}_{n}(z ; s, t)$ is the image of $G_{n}(z)$ (see Section 1.6) under the substitutions $u_{\overline{1}} \mapsto t u_{1}, u_{1} \mapsto s u_{2}, u_{i} \mapsto u_{i+1}(i \geq 2)$. Thus by Proposition 1.4(b), we have

$$
G_{D}(w ; s, t)=\sum_{\mathbf{i} \in \mathcal{R}(w)} s^{\ell_{1}(\mathbf{i})} t^{\ell_{\overline{1}}(\mathbf{i})} K_{\Lambda(\mathbf{i})} .
$$

Note that $G_{D}(\bar{w} ; s, t)=G_{D}(w ; t, s)$ and

$$
G_{D}\left(w^{-1} ; s, t\right)\left(z_{1}, \ldots, z_{m}\right)=G_{D}(w ; s, t)\left(z_{m}, \ldots, z_{1}\right) .
$$

Also, if $w \in A_{n-1}$, then $G_{A}(w ; t)=G_{D}(w ; t, s)=G_{D}(w ; t, 0)$.

An immediate consequence of (8.1) and the fundamental lemma of enriched $P$ partitions (see $(6.2)$ ) is the following heap expansion for $G_{D}(w ; s, t)$ (cf. Proposition $6.5)$.

Proposition 8.1. If $P_{1}, \ldots, P_{k}$ are the heaps of the commutativity classes of $\mathcal{R}(w)$ for some $w \in D_{n}$, then we have

$$
G_{D}(w ; s, t)=\sum_{i=1}^{k} s^{\ell_{1}\left(P_{i}\right)} t^{\ell_{\overline{1}}\left(P_{i}\right)} \Delta\left(P_{i}\right),
$$

where $\ell_{1}(P)$ and $\ell_{\overline{1}}(P)$ denote the number 1 's and $\overline{1}$ 's in the labeled poset $P$.

The formal series $G_{D}(w ; s, t)$ need not be symmetric in the variables $z_{1}, z_{2}, \ldots$; for example, one may check that

$$
G_{D}\left(s_{1} s_{2} s_{\overline{1}} s_{2} ; s, t\right)\left(z_{1}, z_{2}\right)=4 s t z_{1} z_{2}\left(z_{1}+z_{2}\right)\left(2 t z_{1}+z_{2}\right) .
$$

On the other hand, it is known (e.g., Lemma 4.24 of [L]) that in the special case $s=t=1 / 2, \bar{G}_{n}(x ; s, t)$ does commute with $\bar{G}_{n}(y ; s, t)$, and thus

$$
G_{D}(w):=G_{D}(w ; 1 / 2,1 / 2)
$$

is a symmetric function of $z_{1}, z_{2}, \ldots$

Corollary 8.2. If $P$ is the heap of any fully commutative $w \in D_{n}$, then we have $\Delta(P)=2^{l} G_{D}(w)$, where $l$ denotes the number of occurrences of 1 and $\overline{1}$ in any reduced word for $w$. In particular, $\Delta(P)$ is symmetric. 
We claim that there is also a special class of elements $w \in D_{n}$ for which $G_{D}(w ; s, t)$ remains symmetric without specializing $s$ and $t$. To explain, let $I$ denote the two-sided ideal of the nil Coxeter ring generated by $u_{1} u_{2} u_{\overline{1}} u_{2}, u_{\overline{1}} u_{2} u_{1} u_{2}$, $u_{2} u_{\overline{1}} u_{2} u_{1}$, and $u_{2} u_{1} u_{2} u_{\overline{1}}$.

Proposition 8.3. We have $\bar{G}_{n}(x ; s, t) \bar{G}_{n}(y ; s, t)=\bar{G}_{n}(y ; s, t) \bar{G}_{n}(x ; s, t) \bmod I$.

Proof. Applying Proposition 3.1(b) with $a=\bar{G}_{n-2}(x ; s, t), b=\bar{G}_{n-2}(y ; s, t), u=$ $u_{n-2}$, and $v=u_{n-1}$, we see that the assertion follows by induction, once the cases $n=2$ and 3 have been established. However the case $n=2$ is trivial, and $n=3$ is equivalent to showing that for every $w \in D_{3}$, either $G_{D}(w ; s, t)(x, y)$ is symmetric in $x$ and $y$, or else some $\mathbf{i} \in \mathcal{R}(w)$ contains the subword $12 \overline{1} 2, \overline{1} 212,2 \overline{1} 21$, or $212 \overline{1}$. Now if $w$ belongs to a proper parabolic subgroup of $D_{3}$ (i.e., $A_{2}, \bar{A}_{2}$, or $D_{2}$ ), then the symmetry of $G_{D}(w ; s, t)(x, y)$ is either trivial or a consequence of the symmetry of the $G_{A}(t)$-family of quasi-symmetric functions. Retaining only one member from each quadruple $\left(w, \bar{w}, w^{-1}, \bar{w}^{-1}\right)$, there remain only three elements with no reduced word containing $12 \overline{1} 2, \overline{1} 212,2 \overline{1} 21$, or $212 \overline{1}$ as a subword; namely, $s_{1} s_{2} s_{\overline{1}}, s_{1} s_{\overline{1}} s_{2}$, and $s_{2} s_{1} s_{\overline{1}} s_{2}$. For these one obtains

$$
\begin{aligned}
G_{D}\left(s_{1} s_{2} s_{\overline{1}} ; s, t\right)(x, y) & =4 \operatorname{stxy}(x+y), \\
G_{D}\left(s_{1} s_{\overline{1}} s_{2} ; s, t\right)(x, y) & =4 s t(x+y)\left(x^{2}+x y+y^{2}\right), \\
G_{D}\left(s_{2} s_{1} s_{\overline{1}} s_{2} ; s, t\right)(x, y) & =4 s t(x+y)^{2}\left(x^{2}+y^{2}\right),
\end{aligned}
$$

each of which is visibly symmetric.

Define $w \in D_{n}$ to be finely symmetric if there is no member of $\mathcal{R}(w)$ containing any of the subwords $12 \overline{1} 2, \overline{1} 212,2 \overline{1} 21$, or $212 \overline{1}$.

Corollary 8.4. If $w$ is finely symmetric, then $G_{D}(w ; s, t)$ is a symmetric function.

Remark 8.5. (a) Proposition 3.1(b) also provides an easy inductive proof of the fact that $\bar{G}_{n}(x ; 1 / 2,1 / 2)$ commutes with $\bar{G}_{n}(y ; 1 / 2,1 / 2)$ (or equivalently, that $G_{D}(w)=$ $G_{D}(w ; 1 / 2,1 / 2)$ is symmetric for all $\left.w \in D_{n}\right)$. One needs only to check the case $n=3$, a routine calculation.

(b) Since $\bar{G}(z ; s, t) \bar{G}(-z ; s, t)=1$, it follows that $G_{D}(w ; s, t)$ satisfies the Pragacz cancellation law (e.g., see [Ste1, §A.3]), and thus is a $\mathbf{Q}[s, t]$-linear combination of Schur $Q$-functions or $P$-functions whenever it is symmetric. In particular, since the definition of $\bar{G}(z ; s, t)$ shows that $G_{D}(w)$ has integer coefficients relative to monomials in the variables $z_{1}, z_{2}, \ldots$, it follows that $G_{D}(w)$ is $P$-integral. (However, $G_{D}(w)$ need not be $Q$-integral.) Also, since $G_{D}(w ; s, t)$ is a $\mathbf{Z}$-linear combination of the quasi-symmetric functions $K_{\Lambda}$ (see (8.1)), it follows from Theorem 3.8 of [Ste1] that if $G_{D}(w ; s, t)$ is symmetric, it must be a $\mathbf{Z}[s, t]$-linear combination of Schur $Q$-functions.

(c) The symmetric functions $G_{D}(w)$ have been studied by both Lam [L] and Billey and Haiman [BH]. For example, Lam and Billey-Haiman both prove that $G_{D}(w)$ is a nonnegative $\mathbf{Z}$-linear combination of Schur $P$-functions.

It is clear that every $A$-stable $w \in D_{n}$ is finely symmetric, since each of the forbidden subwords $12 \overline{1} 2, \overline{1} 212,2 \overline{1} 21$, and $212 \overline{1}$ is braid-equivalent to a word that contains $1 \overline{1}$. In fact, comparing (3.2) and (8.1), the following is an immediate consequence of Theorem 7.4. 
Theorem 8.6. If $w \in D_{n}$ is A-stable, then we have

$$
G_{D}(w ; s, t)=\sum_{N(w) \subseteq K \subseteq L(|w|)} s^{\xi_{+}(w, K)} t^{\xi_{-}(w, K)} G_{B}\left(|w|^{K}\right) .
$$

In particular, given the $Q$-positivity of the symmetric functions $G_{B}(w)$, we see that for the $A$-stable $w \in D_{n}$, the $Q$-function coefficients of $G_{D}(w ; s, t)$ are polynomials with nonnegative coefficients. In fact, as we will shall see below, this holds for every finely symmetric $w \in D_{n}$.

\section{Finely symmetric elements}

In the following, we reserve the notation $A_{n-2}$ specifically for the parabolic subgroup of $D_{n}$ generated by $s_{2}, \ldots, s_{n-1}$.

Lemma 9.1. We have $A_{n-1}=A_{n-2} \dot{\cup} A_{n-2} s_{1} A_{n-2}$.

Proof. In the canonical reduced word for any $w \in A_{n-1}$, the index $n-1$ occurs at most once. Since $s_{i} \mapsto s_{n-i}$ is an automorphism, it follows that $w$ also has a reduced word in which the index 1 appears at most once.

Lemma 9.2. For $w \in D_{n}$, the following are equivalent.

(a) $w \in A_{n-2} s_{1} s_{\overline{1}} A_{n-2}$.

(b) Every $\mathbf{i} \in \mathcal{R}(w)$ has one 1 , one $\overline{1}$, and no 2 occurs between the 1 and the $\overline{1}$.

(c) The canonical reduced word for $w$ has the subword $1 \overline{1}$ and no other 1 or $\overline{1}$.

(d) The one-line form of $w$ has exactly two negative entries, and the first entry is -1 .

Proof. (a) $\Rightarrow$ (b). If $w \in A_{n-2} s_{1} s_{\overline{1}} A_{n-2}$, then there is at least one $\mathbf{i} \in \mathcal{R}(w)$ that meets the conditions of (b). Furthermore, in any such word, there is no opportunity to apply the braid relations $121 \approx 212$ or $\overline{1} 2 \overline{1} \approx 2 \overline{1} 2$. Since the remaining braid relations preserve the number of occurrences of 1 and $\overline{1}$ as well as the relative positions of 1,2 , and $\overline{1}$, it follows that every $\mathbf{i} \in \mathcal{R}(w)$ meets the conditions of (b).

(b) $\Rightarrow$ (c). Let $\left\langle m_{1}, n_{1}\right] \cdots\left\langle m_{r}, n_{r}\right]$ be the canonical reduced word for $w$, and suppose that 1 (resp., $\overline{1}$ ) occurs in the subword $\left\langle m_{i}, n_{i}\right]$ (resp., $\left\langle m_{j}, n_{j}\right]$ ). If $i=j$, then they appear consecutively in the order $1 \overline{1}$, by construction. Otherwise, if (say) $i<j$, then a 2 must occur immediately following the 1 , contrary to the hypotheses of $(\mathrm{b})$.

(c) $\Rightarrow$ (a) is immediate.

(a) $\Leftrightarrow(\mathrm{d})$. If $w=x s_{1} s_{\overline{1}} y$ for some $x, y \in A_{n-2}$, then the first entry of the one-line form of $y$ must be 1 . Therefore, the first two entries of $s_{1} s_{1} y$ are $(-1,-j)$ for some $j>1$, and $w$ is obtained by arbitrarily permuting the entries of $s_{1} s_{1} y$ in positions beyond the first. Thus (d) holds. Reversing this argument proves the converse.

We remark that it is not possible to characterize the members of the double coset $A_{n-2} s_{1} s_{\overline{1}} A_{n-2}$ in terms of pattern avoidance. Indeed, every pattern involving positive terms occurs in some member of this double coset. However, it contains no member of $A_{n-1}$ and yet members of $A_{n-1}$ have only positive entries.

On the other hand, if we include the double cosets containing the remaining members of $D_{2}$ (i.e., $1, s_{1}$, and $s_{\overline{1}}$ ), it is possible to give both pattern-avoidance and forbidden subword characterizations.

Theorem 9.3. For $w \in D_{n}$, the following are equivalent. 
(a) $w \in A_{n-2} D_{2} A_{n-2}$.

(b) Neither $12 \overline{1}$ nor $\overline{1} 21$ occur as subwords of any $\mathbf{i} \in \mathcal{R}(w)$.

(c) $w$ avoids all patterns $(a, b, c)$ such that $b, c<0$, as well as all patterns that are permutations of $( \pm 1,-2,-3)$.

Proof. (a) $\Rightarrow$ (b). If $w \in A_{n-2} s_{1} s_{\overline{1}} A_{n-2}$, then the implication (a) $\Rightarrow$ (b) of Lemma 9.2 shows that neither $12 \overline{1}$ nor $\overline{1} 21$ can appear in any reduced word for $w$. Otherwise, we have $w \in A_{n-1}$ or $w \in \bar{A}_{n-1}$, in which case every reduced word for $w$ omits either $\overline{1}$ or 1 , and hence (b) is trivially satisfied.

$(\mathrm{b}) \Rightarrow(\mathrm{c})$. Towards a contradiction, assume that $w$ has three entries $(a, b, c)$ that fit one of the patterns forbidden by (c), and no reduced word for $w$ contains $12 \overline{1}$ or $\overline{1} 21$. Among all such counterexamples, choose one that minimizes length. Given this, $w$ cannot have four or more negative entries; otherwise, any choice of $s_{j}$ such that $\ell\left(s_{j} w\right)<\ell(w)$ would yield a shorter element with (at least) four negative entries or two negative entries preceded by a positive one, both of which are forbidden. Thus exactly two of $a, b, c$ are negative, and the remaining entries of $w$ are positive.

If $(a, b, c)$ is a permutation of $(1,-2,-3)$, then since every such permutation is forbidden, we must have $\ell\left(s_{j} w\right)>\ell(w)$ for all $j \geq 1$. In other words, the one-line form of $w$ must be increasing. Therefore $(a, b, c)$ must fit the pattern $(-3,-2,1)$ and $a, b$ are the first two entries of $w$. Whether or not the third entry of $w$ is $c$, the fact that the entries increase implies that the first three entries also fit the pattern $(-3,-2,1)$. However in that case, $w$ has a reduced word that begins with $\overline{1} 21$, a contradiction.

The remaining possibility is that $(a, b, c)$ fits a pattern with $a>0$ and $b, c<0$. Since every entry prior to $b$ is positive, we may assume that $a$ is the first entry of $w$. If we permute any pair of entries of $w$ not involving the first, the result will still contain a forbidden pattern. Therefore, minimality requires $\ell\left(s_{j} w\right)>\ell(w)$ for all $j \geq 2$; i.e., the entries beyond the first position of $w$ must increase. Hence, $(a, b, c)$ fits one of the patterns $(1,-3,-2),(2,-3,-1)$, or $(3,-2,-1)$. In the first two cases, we see that $\ell\left(s_{\overline{1}} w\right)<\ell(w)$ and $s_{\overline{1}} w$ still contains a forbidden pattern (contrary to minimality), but in the last case, $w$ has a reduced word that begins with $12 \overline{1}$, a contradiction.

(c) $\Rightarrow$ (a). If $w$ avoids all patterns involving three negative terms, then $w$ has at most two negative entries. If $w$ has none, then $w \in A_{n-1} \subset A_{n-2} D_{2} A_{n-2}$ (Lemma 9.1), so assume that $w$ has exactly two. If -1 is not one the negative entries, then $w$ contains a pattern formed by some permutation of $(1,-2,-3)$, contrary to (c). If the first entry is positive, then $w$ contains a pattern $(a, b, c)$ such that $b, c<0$, again contrary to (c). Thus the negative entries are -1 and $-j$ for some $j>1$ and one of them occurs in the first position. If -1 occurs first, then Lemma 9.2 implies $w \in A_{n-2} s_{1} s_{\overline{1}} A_{n-2}$. Otherwise, we can find $x \in A_{n-2}$ so that the first two entries of the one-line form of $x w$ are $(-j,-1)$. However in that case, $s_{\overline{1}} x w \in A_{n-2}$, and therefore $w \in A_{n-2} s_{\overline{1}} A_{n-2}$.

Recall that $w \in D_{n}$ is finely symmetric if none of $12 \overline{1} 2, \overline{1} 212,2 \overline{1} 21$, and $212 \overline{1}$ occur as subwords of any $\mathbf{i} \in \mathcal{R}(w)$. This clearly does not happen unless $12 \overline{1}$ and $\overline{1} 21$ occur as well, so we obtain the following.

Corollary 9.4. Every $w \in A_{n-2} D_{2} A_{n-2}$ is finely symmetric. 
Remark 9.5. The members of $A_{n-1}$ and $\bar{A}_{n-1}$ are obviously $A$-stable and therefore finely symmetric, so the only "new" finely symmetric elements identified by this result are the members of $A_{n-2} s_{1} s_{\overline{1}} A_{n-2}$. Since Lemma 9.2 implies that every such element $w$ has exactly one occurrence each of 1 and $\overline{1}$ in every reduced word, it follows that

$$
G_{D}(w ; s, t)=4 s t G_{D}(w)
$$

In particular, the $Q$-positivity of $G_{D}(w ; s, t)$ follows from the $Q$-positivity of $G_{D}(w)$.

Theorem 9.6. For $w \in D_{n}$, the following are equivalent.

(a) $w$ is finely symmetric.

(b) $w$ is A-stable or $w \in A_{n-2} D_{2} A_{n-2}$.

(c) $w$ avoids the patterns

$$
\begin{gathered}
( \pm 1,-2,-3),( \pm 1,-3,-2),(-2, \pm 1,-3),(-3, \pm 1,-2), \\
(-2,-3, \pm 1),(2,-3,-1),(3,-1,-2),(2,-1,-3) .
\end{gathered}
$$

Proof. (a) $\Leftrightarrow(\mathrm{b})$. We have already noted that the $A$-stable members of $D_{n}$, as well as the members of $A_{n-2} D_{2} A_{n-2}$, are finely symmetric. Conversely, if $w$ is not $A$-stable and not in $A_{n-2} s_{1} s_{\overline{1}} A_{n-2}$, then by Theorem 7.1 and Lemma 9.2 the subword $1 \overline{1}$ appears in the canonical reduced word $\mathbf{i}$ for $w$, along with at least one other occurrence of either 1 or $\overline{1}$. It follows that $\mathbf{i}$ has a subword of the form $1 \mathbf{i}^{\prime} 1 \overline{1}$, $\overline{1} \mathbf{i}^{\prime} 1 \overline{1}, 1 \overline{1} \mathbf{i}^{\prime} 1$, or $1 \overline{1} \mathbf{i}^{\prime} \overline{1}$, with 1 and $\overline{1}$ not appearing in $\mathbf{i}^{\prime}$. Note that 2 must appear in $\mathbf{i}^{\prime}$; otherwise $\mathbf{i}$ would not be reduced. However in that case, Lemma 9.1 shows that $\mathbf{i}^{\prime}$ is braid-equivalent to some word in which 2 appears exactly once (and 1 and $\overline{1}$ do not occur at all). Since the indices $>2$ commute with 1 and $\overline{1}$, it follows that $1 \mathbf{i}^{\prime} 1 \overline{1}$ is braid-equivalent to a word containing $121 \overline{1} \approx 212 \overline{1}$, and hence $w$ could not be finely symmetric. (The other cases are similar.)

(b) $\Rightarrow(\mathrm{c})$. If $w$ is $A$-stable, then $w$ avoids the patterns $( \pm 1,-2)$ (Theorem 7.1). Hence $w$ also avoids the patterns listed in (c), since each of them fits either $(1,-2)$ or $(-1,-2)$. Also, any member of $A_{n-2} D_{2} A_{n-2}$ avoids the patterns listed in Theorem 9.3 , and hence also the patterns of (c), since the latter are a subset of the former.

$(\mathrm{c}) \Rightarrow(\mathrm{b})$. Assume $w$ has at least two negative entries; otherwise $w$ is clearly $A$-stable.

Case 1: $w$ avoids $(-1,-2)$. In this case, the negative terms must appear in increasing order, so if the pattern $(1,-2)$ occurs, then one of the patterns $(-3,1,-2)$, $(1,-3,-2)$, or $(2,-3,-1)$ also occurs. However, each of these patterns is explicitly forbidden by (c). Thus $w$ avoids $( \pm 1,-2)$, and hence is $A$-stable (Theorem 7.1).

Case 2: $w$ contains the pattern $(-1,-2)$. In this case, $w$ must contain exactly two negative terms, since the only pattern among the permutations of $(-1,-2,-3)$ that is not forbidden is $(-3,-2,-1)$. If the first entry of $w$ is positive, then one of the patterns $(1,-2,-3),(2,-1,-3)$, or $(3,-1,-2)$ occurs, contrary to (c). If the first entry of $w$ is negative but not -1 , then one of the patterns $(-2,1,-3)$ or $(-2,-3,1)$ occurs, again contrary to (c). Hence the first entry of $w$ is -1 , so $w \in A_{n-2} s_{1} s_{\overline{1}} A_{n-2}$ by Lemma 9.2 .

Proposition 9.7. There are

(a) $\frac{1}{2}(n+1) !+(n-1)(n-1)$ ! finely symmetric members of $D_{n}$, 
(b) $(3 n-2)(n-1)$ ! members of $A_{n-2} D_{2} A_{n-2}$, and

(c) $(2 n-1)(n-1)$ ! A-stable members of $A_{n-2} D_{2} A_{n-2}$.

Proof. The description in Lemma 9.2(d) shows that the double coset $A_{n-2} s_{1} s_{\overline{1}} A_{n-2}$ has $(n-1)(n-1)$ ! members. Thus (a) follows from Theorem 9.6 and Corollary 7.3. Obviously no member of this double coset is $A$-stable, and the remaining members of $A_{n-2} D_{2} A_{n-2}$ consist of $A_{n-1} \cup \bar{A}_{n-1}$. The latter has cardinality $2(n !)-(n-1)$ !, yielding (c); restoring the $(n-1)(n-1)$ ! members of $A_{n-2} s_{1} s_{\overline{1}} A_{n-2}$ yields (b).

Define $w \in D_{n}$ to be $\ell_{ \pm 1}$-invariant if $\ell_{ \pm 1}(\mathbf{i})=\ell_{1}(\mathbf{i})+\ell_{\overline{1}}(\mathbf{i})$ is independent of the choice of $\mathbf{i} \in \mathcal{R}(w)$. Since the only braid relations that affect $\ell_{1}(\mathbf{i})$ or $\ell_{\overline{1}}(\mathbf{i})$ are $121 \approx 212$ and $\overline{1} 2 \overline{1} \approx 2 \overline{1} 2$, it follows that $w$ is $\ell_{ \pm 1}$-invariant if and only if neither 212 nor $2 \overline{1} 2$ occur as subwords of any $\mathbf{i} \in \mathcal{R}(w)$. In particular, every $\ell_{ \pm 1}$-invariant element is finely symmetric.

We remark that the $\ell_{ \pm 1}$-invariant members of $D_{n}$ and $A_{n-2} D_{2} A_{n-2}$, and the $A$-stable portions thereof, cannot be characterized in terms of pattern avoidance. To prove this, note that since $A$-stability and membership in $D_{n}, A_{n-2} D_{2} A_{n-2}$ and $A_{n-1}$ can be characterized by pattern avoidance, and each contains $A_{n-1}$, it suffices merely to show that $\ell_{ \pm 1}$-invariance in $A_{n-1}$ cannot be characterized by pattern avoidance. For this, consider the one-line form of $w=s_{2} s_{1} s_{2} \in D_{3}$, i.e., $(3,2,1)$. Since $w$ is not $\ell_{ \pm 1}$-invariant, $(3,2,1)$ must be a forbidden pattern for $\ell_{ \pm 1^{-}}$ invariance in $A_{n-1}$, if a set of such patterns exists. However $w^{\prime}=s_{2} s_{3} s_{2} \in D_{4}$ has one-line form $(1,4,3,2)$, so it contains the pattern $(3,2,1)$ and yet is clearly $\ell_{ \pm 1 \text {-invariant. }}$

Proposition 9.8. For $w \in D_{n}$, the following are equivalent.

(a) $w$ is A-stable and $\ell_{ \pm 1}$-invariant.

(b) $N(w)=L(|w|)$.

(c) $\sigma \mathcal{R}(w)=\mathcal{R}(x)$ for some $x \in B_{n-1}$.

(d) In the canonical reduced word for $w$, the subword $1 \overline{1}$ does not appear, and the occurrences of 1 and $\overline{1}$ alternate.

Proof. (a) $\Rightarrow(\mathrm{b})$. If $w$ is $A$-stable, then we have $N(w) \subseteq L(|w|)$ (Corollary 7.2). Also, Theorem 7.4 shows that there exist $\mathbf{i}, \mathbf{i}^{\prime} \in \mathcal{R}(w)$ such that $\ell_{ \pm 1}(\mathbf{i})=\# N(w)-1$ and $\ell_{ \pm 1}\left(\mathbf{i}^{\prime}\right)=\# L(|w|)-1$. Thus if $w$ is $\ell_{ \pm 1}$-invariant, $\# N(w)=\# L(|w|)$ and (b) follows.

(b) $\Rightarrow(\mathrm{c})$. If $N(w)=L(|w|)$ then $w$ is $A$-stable (Corollary 7.2). Apply Theorem $7.4(\mathrm{~b})$.

$(\mathrm{c}) \Rightarrow(\mathrm{d})$. Given that $\sigma \mathcal{R}(w)$ only contains reduced words for $B_{n-1}$, the subword $1 \overline{1}$ cannot appear in the canonical (or any) reduced word $\mathbf{i}$ for $w$. If there were (say) a subword $1 \mathbf{i}^{\prime} 1$ of $\mathbf{i}$ such that neither 1 nor $\overline{1}$ occurs in $\mathbf{i}^{\prime}$, then by Lemma $9.1,1 \mathbf{i}^{\prime} 1$ would be braid-equivalent to some word in which 1 occurs exactly once (and $\overline{1}$ not at all). The number of occurrences of 0 in the $\sigma$-images of these words therefore varies. On the other hand, the hypothesis $\sigma \mathcal{R}(w)=\mathcal{R}(x)$ implies that there are $\ell_{0}(x)$ occurrences of 0 in every member of $\sigma \mathcal{R}(w)$, a contradiction.

$(\mathrm{d}) \Rightarrow(\mathrm{a})$. If $1 \overline{1}$ does not occur in the canonical reduced word $\mathbf{i}$ for $w$, then $w$ is $A$ stable (Theorem 7.1), and $\sigma(\mathbf{i})$ is the canonical reduced word for some top element $x \in B_{n-1}$ (Theorem 4.1). Given that the occurrences of 1 and $\overline{1}$ alternate in $\mathbf{i}$, it follows that if $w$ failed to be $\ell_{ \pm 1}$-invariant, there would exist a sequence of braid relations not involving $1 \overline{1} \approx \overline{1} 1,121 \approx 212$, or $\overline{1} 2 \overline{1} \approx 2 \overline{1} 2$ that transforms $\mathbf{i}$ into a reduced word $\mathbf{j}$ containing 212 or $2 \overline{1} 2$. (Each allowed transformation preserves the 
property of alternating 1's and $\overline{1}$ 's, so 212 or $2 \overline{1} 2$ must occur before 121 or $\overline{1} 2 \overline{1}$.) The $\sigma$-images of these transformations are valid for $B_{n-1}$, so $\sigma(\mathbf{j}) \in \mathcal{R}(x)$. However $\sigma(212)=\sigma(2 \overline{1} 2)=101$ is a subword of $\sigma(\mathbf{j})$, which by Theorem 4.1 contradicts the fact that $x$ is a top element.

Remark 9.9. (a) Suppose that $w \in D_{n}$ is $\ell_{ \pm 1}$-invariant but not $A$-stable. Of course $w$ must be finely symmetric, so $w \in A_{n-2} s_{1} s_{1} A_{n-2}$ by Theorem 9.6. However in that case, Lemma 9.2 shows that every reduced word for $w$ has one 1 and one $\overline{1}$, and hence is $\ell_{ \pm 1}$-invariant. In other words, the $\ell_{ \pm 1}$-invariant members of $D_{n}$ are the elements described in Proposition 9.8, together with the members of the double coset $A_{n-2} s_{1} s_{\overline{1}} A_{n-2}$.

(b) For any $\ell_{ \pm 1}$-invariant $w \in D_{n}$, the absence of the subwords 212 and $2 \overline{1} 2$ shows that not only is $\ell_{ \pm 1}(\cdot)$ constant on $\mathcal{R}(w)$, but in fact $\ell_{1}(\cdot)$ and $\ell_{\overline{1}}(\cdot)$ are constant as well. Hence the notations $\ell_{1}(w)$ and $\ell_{\overline{1}}(w)$ are unambiguous. In case $w$ is also $A$-stable, Theorem 7.4 and Proposition $9.8(\mathrm{~d})$ show that

$$
\ell_{1}(w)=\frac{1}{2}(\# L(|w|)-1+\xi), \quad \ell_{\overline{1}}(w)=\frac{1}{2}(\# L(|w|)-1-\xi),
$$

where $\xi$ is the sign of the first entry of $w$ when $\# L(w)$ is even, and 0 otherwise.

(c) If $w \in D_{n}$ is $\ell_{ \pm 1}$-invariant and $A$-stable, then the element $x \in B_{n-1}$ appearing in part (c) of Proposition 9.8 is $\operatorname{top}(|w|)$ (cf. Remark 7.7(a)). If we restrict our attention to the $\ell_{ \pm 1}$-invariant elements $w \in A_{n-1}$, the range of the map $w \mapsto$ $\operatorname{top}(w)$ consists of those elements with exactly one term in the decomposition of Corollary 2.5, i.e., the members of $B_{n-1}$ that are top-and-bottom elements. In other words, there is a bijection between the top-and-bottom elements of $B_{n}$ and the $\ell_{ \pm 1}$-invariant members of $A_{n}$.

Proposition 9.10. There are

(a) $(3 n-2)(n-1)$ ! $\ell_{ \pm 1}$-invariant members of $D_{n}$,

(b) $(2 n-1)(n-1)$ ! A-stable $\ell_{ \pm 1}$-invariant members of $D_{n}$,

(c) $(n-1) !(n+2 H(n-1)) \quad \ell_{ \pm 1}$-invariant members of $A_{n-2} D_{2} A_{n-2}$, and

(d) $(n-1) !(1+2 H(n-1))$ A-stable $\ell_{ \pm 1}$-invariant members of $A_{n-2} D_{2} A_{n-2}$.

Proof. For (b), Proposition 9.8 shows that we can construct the canonical reduced word for any $A$-stable $\ell_{ \pm 1}$-invariant $w \in D_{n}$ by selecting any of the $n$ ! canonical reduced words for $A_{n-1}$, and then replacing every other occurrence of 1 with $\overline{1}$. Assuming there is at least one occurrence of 1 , this can be done in two ways, for a total of $2(n !)-(n-1)$ !.

For (a) recall from Remark 9.9(a) that the $\ell_{ \pm 1}$-invariant members of $D_{n}$ that are not $A$-stable are the members of $A_{n-2} s_{1} s_{\overline{1}} A_{n-2}$. We know from the proof of Proposition 9.7 that this double coset has $(n-1)(n-1)$ ! members.

We know that there are $(n-1) !(1+H(n-1)) \ell_{ \pm 1}$-invariant members of $A_{n-1}$, by Remark 9.9(c) and Proposition 4.3, and hence $(n-1) !(1+2 H(n-1)) \ell_{ \pm 1}$-invariant members of $A_{n-1} \cup \bar{A}_{n-1}$, since every member of $A_{n-1} \cap \bar{A}_{n-1}$ is $\ell_{ \pm 1}$-invariant. This yields (d), and restoring the $(n-1)(n-1)$ ! members of $A_{n-2} s_{1} s_{\overline{1}} A_{n-2}$ yields (c).

\section{Full commutativity}

The equivalence of (a) and (c) in the following has also been obtained by Fan $[\mathrm{F}$, $\S 7]$, although his choice of coordinates is not the same as ours.

Theorem 10.1. For $w \in D_{n}$, the following are equivalent. 
(a) $w$ is fully commutative.

(b) In the canonical reduced word $\left\langle m_{1}, n_{1}\right] \cdots\left\langle m_{r}, n_{r}\right]$ for $w$, the occurrences of 1 and $\overline{1}$ alternate, and either

(1) $m_{1}>\cdots>m_{s}>\left|m_{s+1}\right|=\cdots=\left|m_{r}\right|=1$ for some $s \leq r$, or

(2) $m_{1}>\cdots>m_{r-1}>-m_{r} \geq 0, m_{r-1}>1$, and $m_{r} \neq-1$.

(c) $w$ avoids all patterns $(a, b, c)$ such that $|a|>b>c$ or $-b>|a|>c$.

Proof. (a) $\Rightarrow(\mathrm{b})$. If $w$ is fully commutative, then $w$ is $\ell_{ \pm 1}$-invariant. If $w$ is $A$-stable as well, then (i) the occurrences of 1 and $\overline{1}$ in the canonical reduced word for $w$ must alternate, and (ii) $\sigma \mathcal{R}(w)=\mathcal{R}(x)$ for some $x \in B_{n-1}$, by Proposition 9.8. In fact $x$ must be a fully commutative top element, since otherwise there would be a subword $i(i-1) i$ (with $i \geq 1$ ) appearing in some $\mathbf{i} \in \mathcal{R}(x)$, contradicting the fact that $w$ is fully commutative. Hence the canonical reduced word $\left[m_{1}^{\prime}, n_{1}^{\prime}\right] \cdots\left[m_{r}^{\prime}, n_{r}^{\prime}\right]$ for $x$ satisfies

$$
m_{1}^{\prime}>\cdots>m_{s}^{\prime}>m_{s+1}^{\prime}=\cdots=m_{r}^{\prime}=0
$$

for some $s \leq r$, by Corollary 5.6(b). Any $\sigma$-preimage of this word satisfies (1), and is necessarily the canonical reduced word for some member of $D_{n}$.

Otherwise, if $w$ is not $A$-stable, then $w \in A_{n-2} s_{1} s_{\overline{1}} A_{n-2}$ (see Remark 9.9(a)). It follows that the subword $1 \overline{1}$ occurs in some factor $\left\langle m_{i}, n_{i}\right]$ of the canonical reduced word $\mathbf{i}$ for $w$, and there are no other occurrences of 1 or $\overline{1}$, by Lemma 9.2. Let

$$
\mathbf{i}^{\prime}=\left\langle 2, n_{i}\right]\left\langle m_{i+1}, n_{i+1}\right] \cdots\left\langle m_{r}, n_{r}\right]=\left[2, n_{i}\right]\left[m_{i+1}, n_{i+1}\right] \cdots\left[m_{r}, n_{r}\right]
$$

denote the subword of $\mathbf{i}$ formed by every term following the unique occurrence of $1 \overline{1}$. The word $\mathbf{i}^{\prime}$ is the canonical reduced word for some (necessarily fully) commutative member of the parabolic subgroup of type $A$ generated by $s_{2}, \ldots, s_{n-2}$. Since the first term of $\mathbf{i}^{\prime}$ is 2 (or $\mathbf{i}^{\prime}$ is empty), Corollary 5.8 shows that this is possible only if $i=r$. Therefore, $m_{1}, \ldots, m_{r-1}>1, m_{r} \leq 0$, and $m_{r} \neq-1$.

Now let $m$ be the leading term of $\left\langle m_{r}, n_{r}\right]$; i.e., $m=-m_{r}$ (if $m_{r}<-1$ ) or $m=1$ (if $m_{r}=0$ ), and let

$$
\mathbf{i}^{\prime \prime}=\left\langle m_{1}, n_{1}\right] \cdots\left\langle m_{r-1}, n_{r-1}\right] m=\left[m_{1}, n_{1}\right] \cdots\left[m_{r-1}, n_{r-1}\right][m, m]
$$

be the subword of $\mathbf{i}$ obtained by deleting all terms beyond the first term of $\left\langle m_{r}, n_{r}\right]$. Since $n_{r-1}>n_{r} \geq\left|m_{r}\right|$ in every canonical reduced word, it follows that $\mathbf{i}^{\prime \prime}$ is a canonical reduced word for some (necessarily fully commutative member of $A_{n-1}$. By Corollary 5.8, it follows that $m_{1}>\cdots>m_{r-1}>m \geq 1$.

(c) $\Rightarrow(\mathrm{a})$. Arguing by contradiction, it suffices to prove the following.

(i) If $w$ has a reduced word that begins with $2 \overline{1} 2$ or $i(i-1) i$ for some $i>1$, then $w$ contains one of the patterns forbidden by (c).

(ii) If $\ell\left(s_{j} w\right)>\ell(w)$ and $w$ contains a pattern forbidden by (c), then so does $s_{j} w$.

Given the hypothesis of (i), $w$ has reduced words beginning with either of $\overline{1}$ and 2 , or $i-1$ and $i$. In the former case, the one-line form of $w$, say $\left(w_{1}, \ldots, w_{n}\right)$, satisfies $-w_{1}>w_{2}>w_{3}$, and in the latter case we have $w_{i-1}>w_{i}>w_{i+1}$. In either case, $w$ contains one of the forbidden patterns.

For (ii), suppose $\ell\left(s_{j} w\right)>\ell(w)$ and that the one-line form of $w$ has a subsequence $(a, b, c)$ such that $|a|>b>c$ or $-b>|a|>c$. If $j \geq 1$ then the same is true of $s_{j} w$, by the same argument used in the proof of the implication (c) $\Rightarrow$ (a) for Theorem 5.1. We therefore consider only the case $j=\overline{1}$. If $b$ does not occur in the second position of $w$, then $s_{\overline{1}} w$ has a subsequence of the form $( \pm a, b, c)$, contrary to (c). Otherwise, $a$ and $b$ occur in the first two positions of $w$, and $\left(a^{\prime}, b^{\prime}, c^{\prime}\right)=(-b,-a, c)$ 
is a subsequence of $s_{\overline{1}} w$. We also have $a+b>0$ (since $\left.\ell\left(s_{\overline{1}} w\right)>\ell(w)\right)$, so the subsequence $(a, b, c)$ must satisfy $a>b>c$ or $-b>-a>c$. In the latter case, we obtain $a^{\prime}>b^{\prime}>c^{\prime}$ and hence $\left|a^{\prime}\right|>b^{\prime}>c^{\prime}$, which is forbidden by (c). In the former case, we obtain $-b^{\prime}>-a^{\prime}>c^{\prime}$. However $a+b>0$ also implies $-b^{\prime}>a^{\prime}$, so we have $-b^{\prime}>\left|a^{\prime}\right| \geq-a^{\prime}>c^{\prime}$. Hence $-b^{\prime}>\left|a^{\prime}\right|>c^{\prime}$, which is also forbidden by $(\mathrm{c})$.

(b) $\Rightarrow(\mathrm{c})$. Let $\mathbf{i}=\left\langle m_{1}, n_{1}\right] \cdots\left\langle m_{r}, n_{r}\right]$ denote the canonical reduced word for $w$.

Case 1: i satisfies (1). In this case, $w$ is $A$-stable and $\ell_{ \pm 1}$-invariant, by Proposition 9.8. In particular, Theorem 7.1 implies that $w$ avoids the patterns $( \pm 1,-2)$, and hence all patterns $(a, b, c)$ such that $-b>|a|>c$ (or even $-b>|a|)$ ). Furthermore, among the patterns $(a, b, c)$ such that $|a|>b>c$ (see (5.2)), the only ones that manage to avoid $( \pm 1,-2)$ are the patterns $( \pm 3,2, \pm 1)$. Hence for this case, it suffices to prove that $w$ avoids the patterns $( \pm 3,2, \pm 1)$.

For this, we first note that $\sigma(\mathbf{i})$ is the canonical reduced word for some fully commutative top element $x \in B_{n-1}$, by Corollary 5.6. In particular (again by Corollary 5.6), $x$ avoids the patterns $( \pm 1,-2)$ and $( \pm 3,2, \pm 1)$. Towards a contradiction, suppose that $(a, b, c)$ is a subsequence of the one-line form of $w$ that fits one of the patterns $( \pm 3,2, \pm 1)$ (i.e., $|a|>b>|c|)$. Since $x$ is a top element, $x$ is obtained by unbumping the entries of $|w|$ at the positions where left-minima occur. Therefore if $b$ appears to the right of \pm 1 in $w$, no unbumping affects $b$ and $c$ and $( \pm(a-1), b-1, c-1)$ is a subsequence of $x$ fitting one of the patterns $( \pm 3,2,1)$, a contradiction. Otherwise, let $a_{1}$ (resp., $a_{2}$ ) be the left-minimum of $|w|$ immediately preceding (resp., following) $b$ in $|w|$. Note that $b$ itself cannot be a left-minimum, since $b>0$ and $N(w)=L(|w|)$ (Proposition 9.8). Therefore to obtain $x$ from $|w|$, $a_{1}$ unbumps $a_{2}$, replacing it with $-\left(a_{1}-1\right)$. In particular, $\left(b-1,-\left(a_{1}-1\right)\right)$ is a subsequence of the one-line form of $x$. Since $x$ avoids $(1,-2)$, this requires $a_{1}<b$. Now if no unbumping affects $a$, then $a>0$ and $\left(a-1, b-1,-\left(a_{1}-1\right)\right)$ is a subsequence of $x$ fitting the pattern $(3,2,-1)$, a contradiction. Otherwise, if $|a|$ is unbumped, then $|a|$ appears to the left of $a_{1}$ (since $\left.|a|>b>a_{1}\right)$, and $\left(-|a-1|, b-1,-\left(a_{1}-1\right)\right.$ ) is a subsequence of $x$ fitting the pattern $(-3,2,-1)$, a contradiction.

Case 2: $\mathbf{i}$ satisfies (2). In this case, $1 \overline{1}$ is a subword of $\mathbf{i}$ and there are no other occurrences of 1 or $\overline{1}$. Let $\mathbf{j}$ be the word obtained by deleting one of the two (consecutive) occurrences of 0 from $\sigma(\mathbf{i})$. The constraints of (2) imply

$$
\mathbf{j}=\left[m_{1}-1, n_{1}-1\right] \cdots\left[m_{r-1}-1, n_{r-1}-1\right]\left[-(m-1), n_{r}-1\right],
$$

where $m$ denotes the leading term of $\left\langle m_{r}, n_{r}\right]$. Since $m_{1}>\cdots>m_{r-1}>m \geq 1$, it follows that $\mathbf{j}$ is the canonical reduced word for some $x \in B_{n-1}$ of the type described in Corollary 5.7(b), and is therefore fully commutative. By Lemma 9.2, the one-line form of $w$ has exactly two negative entries, and the first entry is -1 . The effect of $s_{1} s_{\overline{1}}$ on one-line forms of members of $D_{n}$ is to negate the first two coordinates, whereas the effect of $s_{0}$ on $B_{n}$ is to negate only the first coordinate. The remaining generators act as adjacent transpositions on $B_{n}$ and $D_{n}$. It follows that if we delete the initial -1 from $w$ and replace each remaining $i$ (resp., $-i$ ) such that $i \geq 2$ with $i-1$ (resp., $-(i-1))$, we obtain the one-line form of $x$. Therefore, if $(a, b, c)$ is a subsequence of the one-line form of $w$ such that $|a|>b>c$ or $-b>|a|>c$, then $a$ is the first entry of $w$; otherwise there would be subsequence of $x$ fitting the same pattern, contrary to Theorem 5.1. However the first entry is -1 , so $|a|>b>c$ or $-b>|a|>c$ would both imply the impossibility $b, c<0$. 
Since $( \pm 3,2, \pm 1)$ are the only $( \pm 1,-2)$-avoiding patterns $(a, b, c)$ such that $|a|>$ $b>c$ or $-b>|a|>c$, we obtain the following.

Corollary 10.2. For $w \in D_{n}$, the following are equivalent.

(a) $w$ is fully commutative and A-stable.

(b) In the canonical reduced word $\left\langle m_{1}, n_{1}\right] \cdots\left\langle m_{r}, n_{r}\right]$ for $w$, the occurrences of 1 and $\overline{1}$ alternate and $m_{1}>\cdots>m_{s}>\left|m_{s+1}\right|=\cdots=\left|m_{r}\right|=1$ for some $s \leq r$.

(c) $w$ avoids the patterns $( \pm 1,-2)$ and $( \pm 3,2, \pm 1)$.

Similarly, by selecting the patterns $(a, b, c)$ such that $|a|>b>c$ or $-b>|a|>c$ that are not eliminated by Theorem 9.3 , we obtain

Corollary 10.3. For $w \in A_{n-2} D_{2} A_{n-2}$, the following are equivalent.

(a) $w$ is fully commutative.

(b) The canonical reduced word $\left\langle m_{1}, n_{1}\right] \cdots\left\langle m_{r}, n_{r}\right]$ for $w$ satisfies $m_{1}>\cdots>m_{r-1}>\max \left(\left|m_{r}\right|, 1\right)$.

(c) $w$ avoids the patterns $( \pm 3,2, \pm 1),(2,-3,1),(3,1,-2)$, and $(2,1,-3)$.

Part (a) of the following has also been obtained by Fan (Proposition 3 of $[\mathrm{F}]$ ).

Proposition 10.4. There are

(a) $\frac{1}{2}(n+3) C(n)-1$ fully commutative members of $D_{n}$.

(b) $\frac{1}{2}\left(\begin{array}{c}2 n \\ n\end{array}\right)$ fully commutative A-stable members of $D_{n}$.

(c) $3 C(n)-C(n-1)-1$ fully commutative members of $A_{n-2} D_{2} A_{n-2}$.

(d) $2 C(n)-C(n-1)$ fully commutative A-stable members of $A_{n-2} D_{2} A_{n-2}$.

Proof. For $w \in B_{n}$, let $w^{\prime} \in B_{n}$ be the element obtained by changing the sign of the entry \pm 1 in the one-line form of $w$. Exactly one member of each pair $\left(w, w^{\prime}\right)$ is the one-line form of a member of $D_{n}$. Furthermore, $w$ avoids the patterns $( \pm 1,-2)$ and $( \pm 3,2, \pm 1)$ if and only if the same is true of $w^{\prime}$. Comparing Corollary 10.2 with Corollary 5.6, we deduce that there are half as many fully commutative $A$ stable members of $D_{n}$ as there are fully commutative top elements in $B_{n}$. Applying Proposition 5.9(b), we obtain (b).

For (d), recall that $A_{n-1} \cup \bar{A}_{n-1}$ is the set of $A$-stable members of $A_{n-2} D_{2} A_{n-2}$. We know that $A_{n-1}$ and $\bar{A}_{n-1}$ each have $C(n)$ fully commutative elements, and their intersection (being $\left.A_{n-2}\right)$ has $C(n-1)$ such elements, yielding a total of $2 C(n)-C(n-1)$.

By Theorem 10.1, the canonical reduced words $\left\langle m_{1}, n_{1}\right] \cdots\left\langle m_{r}, n_{r}\right]$ for the fully commutative members of $A_{n-2} s_{1} s_{\overline{1}} A_{n-2}$ are characterized by the relations

$$
m_{1}>\cdots>m_{r-1}>m \geq 1, m_{r} \leq 0, m_{r} \neq-1,
$$

where $m$ denotes the leading term of $\left\langle m_{r}, n_{r}\right]$. Also, given that $m_{r}=0$ or $m_{r}<-1$, the leading term of $\left\langle m_{r}, n_{r}\right]$ uniquely determines $m_{r}$ as well. Comparing this with Theorem 5.1, we see that there is a one-to-one correspondence between these words and the canonical reduced words for the fully commutative members of $B_{n}$ that are not top elements. There are $C(n)-1$ of the latter, by parts (a) and (b) of Proposition 5.9.

Since $A_{n-2} s_{1} s_{\overline{1}} A_{n-2}$ is the set of $\ell_{ \pm 1}$-invariant members of $D_{n}$ that are not $A$-stable, it follows that there are $\frac{1}{2}\left(\begin{array}{c}2 n \\ n\end{array}\right)+C(n)-1=\frac{1}{2}(n+3) C(n)-1$ fully commutative members of $D_{n}$ (yielding (a)), and $2 C(n)-C(n-1)+C(n)-1$ fully commutative members of $A_{n-2} D_{2} A_{n-2}$, yielding (c). 
Let $\left\langle m_{1}, n_{1}\right] \cdots\left\langle m_{r}, n_{r}\right]$ be the canonical reduced word for some $A$-stable fully commutative $w \in D_{n}$, and let $s$ be the largest index such that $m_{s} \geq 2$. We define the shape of $w$ to be the shifted shape $\lambda / \mu$, where $\lambda=\left(n_{1}, \ldots, n_{r}\right)$ and $\mu=\left(m_{1}-1, \ldots, m_{s}-1\right)$.

The $\mu=\varnothing$ case in part (b) of the following is equivalent to Proposition 3.13 of $[\mathrm{BH}]$.

Proposition 10.5. If $w \in D_{n}$ is fully commutative, A-stable and of shape $\lambda / \mu$, then

(a) The heap of $w$ is isomorphic to $D_{\lambda / \mu}^{\prime}$ (as a labeled poset).

(b) $G_{D}(w)=2^{-(\ell(\lambda)-\ell(\mu))} \Delta\left(D_{\lambda / \mu}^{\prime}\right)=P_{\lambda / \mu}$.

Proof. Let $\mathbf{i}$ be the canonical reduced word for $w$. As we have noted previously, $\sigma(\mathbf{i})$ is the canonical reduced word for some fully commutative top element $x \in B_{n-1}$. In fact $x$ and $w$ have the same shape, so by Proposition 6.1, the heap of $x$ is isomorphic to $D_{\lambda / \mu}^{\prime}$. Since there is no reduced word for $w$ in which 1 and $\overline{1}$ appear consecutively, it follows that the heaps of $\mathbf{i}$ and $\sigma(\mathbf{i})$ are isomorphic as labeled posets (yielding (a)). Furthermore, we have $\ell_{ \pm 1}(w)=\ell_{0}(x)=\ell(\lambda)-\ell(\mu)$ (the number of cells on the main diagonal of $D_{\lambda / \mu}^{\prime}$ ), so (b) follows from Corollary 6.6 and Corollary 8.2.

Now consider the heap of some fully commutative $w \in A_{n-2} s_{1} s_{\overline{1}} A_{n-2}$; by Theorem 10.1, we know that the only fully commutative members of $D_{n}$ that are not $A$-stable are of this type. Furthermore, if $\mathbf{i}=\left\langle m_{1}, n_{1}\right] \cdots\left\langle m_{r}, n_{r}\right]$ is the canonical reduced word for $w$, then we have $m_{1}>\cdots>m_{r-1}>m \geq 1$, where $m$ denotes the leading term of $\left\langle m_{r}, n_{r}\right]$. We define the shape of $w$ to be the shape of the fully commutative $A$-stable element whose canonical reduced word is

$$
\mathbf{j}=\left\langle m_{1}, n_{1}\right] \cdots\left\langle m_{r-1}, n_{r-1}\right]\left\langle m, n_{r}\right] .
$$

That is, the shape of $w$ is $\lambda / \mu$, where $\lambda=\left(n_{1}, \ldots, n_{r}\right)$ and $\mu=\left(m_{1}-1, \ldots, m_{r-1}-\right.$ $1, m-1)$.

We obtain $\mathbf{i}$ from $\mathbf{j}$ by replacing the unique occurrence of the smallest term $m$ with the word $m \cdots 21 \overline{1} 2 \cdots m$. (In case $m=1$, we replace 1 with $1 \overline{1}$.) It follows that the heap of $\mathbf{i}$ can be obtained from the heap of $\mathbf{j}$ by replacing the unique vertex labeled $m$ with the heap of $m \cdots 21 \overline{1} 2 \cdots m$. The latter is nearly a total order, the only exception being that the vertices labeled 1 and $\overline{1}$ are incomparable.

More explicitly, given a labeled poset $P$ with a unique vertex $x$ labeled $m \geq 1$, define $Y_{m}(P)$ to be the labeled poset obtained from $P$ by replacing $x$ with $2 m$ elements ordered so that

$$
x_{-m}<\cdots<x_{-2}<x_{-1}, x_{1}<x_{2}<\cdots<x_{m} .
$$

The label of $x_{-1}$ is defined to be $\overline{1}$, and all other elements $x_{i}$ are labeled $|i|$. (Compare this with the definition of $I_{m}(P)$ in Section 6.1.)

Summarizing, we have the following.

Proposition 10.6. If $w \in A_{n-2} s_{1} s_{\overline{1}} A_{n-2}$ is fully commutative, then the heap of $w$ is isomorphic to $Y_{m}(P)$, where $P$ is the heap of the fully commutative A-stable element of the same shape as $w$, and $m$ is the smallest label in $P$.

For example, the fully commutative element $w \in D_{8}$ whose canonical reduced word is $\langle 6,8]\langle 4,6]\langle-3,5]$ has shape $865 / 532$ (cf. Figure 1 ). The corresponding fully commutative $A$-stable element has canonical reduced word $\mathbf{j}=\langle 6,8]\langle 4,6]\langle 3,5]$ and 


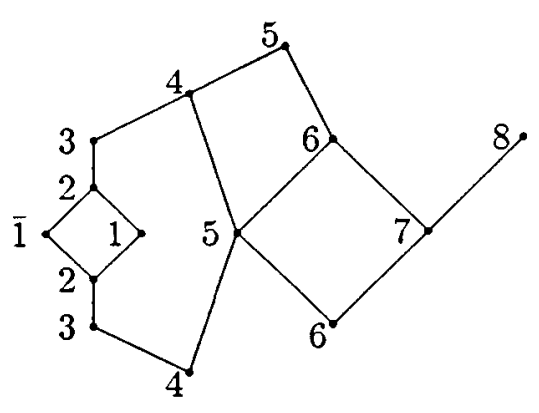

FIGURE 4

smallest term $m=3$. The heap of $w$ is therefore obtained by replacing the vertex labeled 3 in the heap of $\mathbf{j}$ with the heap of $321 \overline{1} 23$ (see Figure 4).

Remark 10.7. By Corollary 8.2, it follows that we obtain a $\Delta$-symmetric poset from any skew diagram $D_{\lambda / \mu}^{\prime}$ with smallest label $m>0$ by applying the operation $Y_{m}$.

\section{The $V$-stable members of $D_{n}$}

Let $\mathbf{i}=i_{1} \cdots i_{l}$ be a reduced word for some $w \in D_{n}$, and set $w_{k}=s_{i_{k+1}} s_{i_{k+2}} \cdots s_{i_{l}}$ for $0 \leq k \leq l$. (In particular, $w_{l}$ is the identity element.) The elements $w_{0}, \ldots, w_{l}$ form a shortest path from $w=w_{0}$ to the identity. We define $V(\mathbf{i})$ to be the set of "1-visitors" along this path, i.e., the set of entries that appear in the first positions of the one-line forms of $\left|w_{0}\right|, \ldots,\left|w_{l}\right|$. It will be convenient to let $\nu(\mathbf{i}):=\# V(\mathbf{i})$.

For example, if $\mathbf{i}=2 \overline{1} 2$, then the one-line forms of $w=w_{0}, w_{1}, w_{2}$ and $w_{3}$ are (in reverse order) $(1,2,3),(1,3,2),(-3,-1,2)$, and $(-3,2,-1)$, so we have $V(\mathbf{i})=\{1,3\}$.

The following result also occurs in the work of Billey-Haiman (Proposition 3.7 of $[\mathrm{BH}])$.

Lemma 11.1. For $w \in D_{n}$ and $\mathbf{i} \in \mathcal{R}(w)$, we have

$$
\#\{\mathbf{j} \in \mathcal{R}(w): \sigma(\mathbf{j})=\sigma(\mathbf{i})\}=2^{\ell_{ \pm 1}(\mathbf{i})-\nu(\mathbf{i})+1} .
$$

Proof. Let $l=\ell_{ \pm 1}(\mathbf{i})$. For any $\mathbf{j} \in \mathcal{R}(w)$, define $\mathbf{j}^{\prime}$ to be the word obtained by replacing each occurrence of $\overline{1}$ with 1 . There is a unique factorization $\mathbf{i}_{0} \mathbf{i}_{1} \cdots \mathbf{i}_{l}$ of $\mathbf{i}^{\prime}$ in which 1 is the last term of $\mathbf{i}_{k}$ for $0 \leq k<l$ and $\mathbf{i}_{l}$ is possibly empty.

For $0 \leq k \leq l$, let $x_{k} \in A_{n-1}$ denote the product of the generators indexed by $\mathbf{i}_{k}$. There is a one-to-one correspondence between the set of words $\mathbf{j} \in \mathcal{R}(w)$ such that $\mathbf{i}^{\prime}=\mathbf{j}^{\prime}$ (or equivalently, $\left.\sigma(\mathbf{i})=\sigma(\mathbf{j})\right)$ and $l$-tuples $\left(t_{1}, \ldots, t_{l}\right)$ taken from $\left\{1, s_{1} s_{\overline{1}}\right\}$ such that

$$
w=x_{0} t_{1} x_{1} t_{2} \cdots x_{l-1} t_{l} x_{l} .
$$

Indeed, one chooses $t_{k}=1$ (resp., $t_{k}=s_{1} s_{\overline{1}}$ ) according to whether the $k$ th occurrence of 1 in $\mathbf{i}^{\prime}$ is in a position where 1 (resp., $\overline{1}$ ) occurs in $\mathbf{j}$.

Thus the objective is to count solutions of (11.1).

For this, note that $D_{n}$ is the semi-direct product of $A_{n-1}$ and the kernel $T$ of the homomorphism $w \mapsto|w|$. In particular, every $w \in D_{n}$ has a (unique) representation $w=|w| \cdot t$ for some $t \in T$. Given any solution of (11.1), we see that $|w|=x_{0} \cdots x_{l}$ 
and the element $t$ is given by

$$
\left(x_{0} \cdots x_{l}\right)^{-1}\left(x_{0} t_{1} x_{1} t_{2} \cdots x_{l-1} t_{l} x_{l}\right)=t_{1}^{y_{1}} t_{2}^{y_{2}} \cdots t_{l}^{y_{l}},
$$

where $y_{k}=x_{k} x_{k+1} \cdots x_{l}$ and $t^{y}=y^{-1} t y$. Conversely, $\left(t_{1}, \ldots, t_{l}\right)$ is a solution of (11.1) whenever $t=t_{1}^{y_{1}} t_{2}^{y_{2}} \cdots t_{l}^{y_{l}}$. However $T$ is abelian, so

$$
\left(t_{1}, \ldots, t_{l}\right) \stackrel{\varphi}{\longmapsto} t_{1}^{y_{1}} t_{2}^{y_{2}} \cdots t_{l}^{y_{l}}
$$

is clearly a group homomorphism $\left\{1, s_{1} s_{\overline{1}}\right\}^{l} \rightarrow T$. It follows that the number of solutions of $(11.1)$ is $\#(\operatorname{Ker} \varphi)=2^{l} / \#(\operatorname{Im} \varphi)$.

To determine the range of $\varphi$, note that for any $y \in A_{n-1},\left(s_{1} s_{\overline{1}}\right)^{y}=y^{-1} s_{1} s_{\overline{1}} y \in T$ acts on $\mathbf{R}^{n}$ by changing the sign of the two coordinates indexed by the first two entries in the one-line form of $y$. Now as the one-line form of $w$ is computed by applying the generators of $\mathbf{i}$ (read from right to left), the entry that appears in the first coordinate changes only when the generator to be applied is $s_{1}$ or $s_{\overline{1}}$. It follows that the members of $V(\mathbf{i})$ are the first entries of $y_{0}, \ldots, y_{l}$; say, $v_{0}, v_{1}, \ldots, v_{l}=1$. (We are not assuming that $v_{0}, \ldots, v_{l}$ are distinct.) Furthermore, in passing from $y_{k}$ to $y_{k-1}$, the entry in the second position of $y_{k}$ moves to the first position of $y_{k-1}$; in other words, the first two entries of $y_{k}$ are $\left(v_{k}, v_{k-1}\right)$. It follows that $\operatorname{Im} \varphi$ is the subgroup of $T$ consisting of all sign changes involving even subsets of coordinates indexed by $V(\mathbf{i})$, a group of order $2^{\nu(\mathbf{i})-1}$.

It is easy to see that for $\mathbf{i} \in \mathcal{R}\left(D_{n}\right), V(\mathbf{i})$ and $\Lambda(\mathbf{i})$ (see (1.2)) depend only on $\mathbf{j}=\sigma(\mathbf{i})$. Hence the use of $\nu(\mathbf{j})$ and $\Lambda(\mathbf{j})$ in the following is unambiguous.

Theorem 11.2. For $w \in D_{n}$, we have

$$
G_{D}(w)=\sum_{\mathbf{j} \in \sigma \mathcal{R}(w)} \frac{1}{2^{\nu(\mathbf{j})-1}} K_{\Lambda(\mathbf{j})} .
$$

Proof. Set $s=t=1 / 2$ in (8.1) and apply Lemma 11.1.

Define $w \in D_{n}$ to be $V$-stable if $V(\mathbf{i})$ does not depend on the choice of $\mathbf{i} \in \mathcal{R}(w)$.

Theorem 11.3. For $w \in D_{n}$, the following are equivalent.

(a) $w$ is $V$-stable.

(b) $\nu(\mathbf{i})=\# V(\mathbf{i})$ is independent of $\mathbf{i} \in \mathcal{R}(w)$.

(c) $L(|w|) \subseteq N(w)$.

Moreover, if $w$ is $V$-stable, then $\nu(\mathbf{i})=\# N(w)$ for all $\mathbf{i} \in \mathcal{R}(w)$.

This result is an immediate corollary of the following.

Lemma 11.4. If $\left(w_{1}, \ldots, w_{n}\right)$ is the one-line form of $w \in D_{n}$, then

$$
N(w) \subseteq\left\{j:\left|w_{j}\right| \in V(\mathbf{i})\right\} \subseteq N(w) \cup L(|w|)
$$

for every $\mathbf{i} \in \mathcal{R}(w)$. Furthermore, both bounds are attained.

Proof. Let $j \in N(w)$; i.e., $w_{j}<0, j=1$, or $\left|w_{j}\right|=1$. If $w_{j}<0$, then $\pm w_{j}$ must be the first entry of some member of the path from $w$ to the identity defined by any $\mathbf{i} \in \mathcal{R}(w)$, since an entry cannot be changed from negative to positive without appearing in the first position. Also, $w_{1}$ and 1 must appear in the first position of the starting and finishing members of the path. Thus in each case, $j \in N(w)$ implies $\left|w_{j}\right| \in V(\mathbf{i})$. 
Next consider some index $j \notin N(w) \cup L(|w|)$; i.e., suppose that $w_{j}$ is a positive entry of $w$ that is not a left-minimum of $|w|$. We claim that if $\ell\left(s_{i} w\right)<\ell(w)$, then the same is true of $s_{i} w$; i.e., $w_{j}$ is a positive entry of $s_{i} w$ that is not a left-minimum. If $s_{i}$ does not change the $j$ th coordinate then there is nothing to prove, so assume $i=j, i=j-1$, or $i=\overline{1}$ and $j=2$. (We cannot have $j=1$, otherwise $w_{j}$ is trivially a left-minimum.) If $i=j$, then $s_{i}$ moves $w_{j}$ to the right and hence it remains a left non-minimum. If $i=j-1$, then $s_{i}$ moves $w_{j}$ to the left. However in that case, $\ell\left(s_{i} w\right)<\ell(w)$ implies $w_{j-1}>w_{j}$, so $w_{j}$ remains a left non-minimum. Finally, if $i=\overline{1}$ and $j=2$, then $\ell\left(s_{i} w\right)<\ell(w)$ implies $w_{1}+w_{2}<0$. However this yields $0<w_{2}<-w_{1}$; thus $w_{j}=w_{2}$ is a left-minimum of $|w|$, a contradiction.

Having proved the claim, it follows by induction on $\ell(w)$ that any positive entry $a$ of $w$ that is not a left-minimum of $|w|$ can never occur as a left-minimum in any member of the path from $|w|$ to the identity defined by i. In particular, no such entry can appear in the first position; i.e., $j \notin N(w) \cup L(|w|)$ implies $\left|w_{j}\right| \notin V(\mathbf{i})$.

Attaining the lower bound. Since $N(w) \subseteq\left\{j:\left|w_{j}\right| \in V(\mathbf{i})\right\}$, it suffices to exhibit some $\mathbf{i} \in \mathcal{R}(w)$ such that $\nu(\mathbf{i}) \leq \# N(w)$. For this we proceed by induction on $\ell(w)$.

Case 1: $w \in A_{n-1}$. In this case, $w$ has no negative entries. If the first entry of $w$ is 1 , then $\# N(w)=1$ and $\nu(\mathbf{i})=1$ for every $\mathbf{i} \in \mathcal{R}(w)$. Otherwise, if 1 occurs in position $j+1 \geq 2$, then $\ell\left(s_{j} w\right)<\ell(w)$. Hence by induction, there exists $\mathbf{j} \in \mathcal{R}\left(s_{j} w\right)$ such that $\nu(\mathbf{j}) \leq \# N\left(s_{j} w\right)$, and by adding $j$ to the beginning of $\mathbf{j}$ we obtain a reduced word $\mathbf{i}$ for $w$. If $j=1$, then the first entry of $s_{j} w$ is 1 , so we obtain $\nu(\mathbf{i})=2$ and $N(w)=\{1,2\}$. On the other hand, if $j>1$ then $\nu(\mathbf{i})=\nu(\mathbf{j})$ and $\# N(w)=\# N\left(s_{j} w\right)=2$. In either case, we obtain $\nu(\mathbf{i}) \leq \# N(w)$.

Case 2: $w \notin A_{n-1}$. In this case, $w$ has two or more negative entries. If the first two entries are negative then $\ell\left(s_{\overline{1}} w\right)<\ell(w)$, so by induction we can find $\mathbf{j} \in \mathcal{R}\left(s_{\overline{1}} w\right)$ so that $\nu(\mathbf{j})=\# N\left(s_{\overline{1}} w\right)$, and adding $\overline{1}$ at the beginning of $\mathbf{j}$ yields a reduced word $\mathbf{i}$ for $w$. Since the second entry of $s_{\overline{1}} w$ is now positive, we have $\# N(w)-\# N\left(s_{\overline{1}} w\right)=1$, unless this second entry is 1 , in which case $\# N(w)=\# N\left(s_{\overline{1}} w\right)$. On the other hand, in passing from $\mathbf{j}$ to $\mathbf{i}$, at most one new entry appears in the first position; i.e., $\nu(\mathbf{i})-\nu(\mathbf{j}) \leq 1$, with equality occurring only if $\left|w_{1}\right|>1$. Thus in either case, we obtain $\nu(\mathbf{i}) \leq \# N(w)$.

The remaining possibility is that $w$ has a positive entry in some position $j \geq 1$, immediately followed by a negative entry. It follows that $\ell\left(s_{j} w\right)<\ell(w)$, so by induction there exists $\mathbf{j} \in \mathcal{R}\left(s_{j} w\right)$ such that $\nu(\mathbf{j}) \leq \# N\left(s_{j} w\right)$, and by adding $j$ to the beginning of $\mathbf{j}$ we obtain a reduced word $\mathbf{i}$ for $w$. Since $w$ and $s_{j} w$ have the same negative entries, we have $\# N(w)=\# N\left(s_{j} w\right)$, unless $j=1$ and $w_{1}>1$, in which case $\# N(w)-\# N\left(s_{j} w\right)=1$. In passing from $\mathbf{j}$ to $\mathbf{i}$, we have $\nu(\mathbf{i})-\nu(\mathbf{j}) \leq 1$, with equality only if a new entry appears in the first position. Since the latter occurs only if $j=1$ and $w_{1}>1$, we again obtain $\nu(\mathbf{i}) \leq \# N(w)$ in either case.

Attaining the upper bound. Since $\left\{j:\left|w_{j}\right| \in V(\mathbf{i})\right\} \subseteq N(w) \cup L(|w|)$, it follows that the upper bound is attained if there is some $\mathbf{i} \in \mathcal{R}(w)$ such that $\nu(\mathbf{i})=$ $\#(N(w) \cup L(|w|))$. In fact, we claim that this occurs when $\mathbf{i}$ is the canonical reduced word for $w$. Proceeding by induction with respect to $n$, let $x_{n} \cdots x_{2}$ be the canonical factorization of $w$, and let $\mathbf{i}^{\prime}$ be the canonical reduced word for $w^{\prime}=x_{n-1} \cdots x_{2}$, a suffix of $\mathbf{i}$.

If $n$ occurs in position $j>1$ of $w$, then the one-line form of $w$ is obtained from $w^{\prime}$ by removing $n$ from the $n$th position (regarding $w^{\prime}$ as a member of $D_{n}$ ) and 
re-inserting it into position $j$. In that case, we claim that

$$
\nu(\mathbf{i})=\nu\left(\mathbf{i}^{\prime}\right)=\#\left(N\left(w^{\prime}\right) \cup L\left(\left|w^{\prime}\right|\right)\right)=\#(N(w) \cup L(|w|)) .
$$

The first equality is a consequence of the fact that in passing from $\mathbf{i}^{\prime}$ to $\mathbf{i}$, the entry $n$ never occupies the first position. The second equality is the induction hypothesis, and the last is a consequence of the fact that since $n$ does not occur in the first position of $w$, it cannot be a left-minimum of $|w|$.

Otherwise, in case $-n$ occurs in any position, or $n$ occurs in the first position of $w$, then $\pm n$ must visit the first position in passing from $\mathbf{i}^{\prime}$ to $\mathbf{i}$, and either a new negative entry occurs in some position beyond the first, or a new left-minimum is created. It follows that the values of $\nu(\mathbf{i})$ and $\#(N(w) \cup L(|w|))$ are increased by 1 relative to the corresponding values for $\mathbf{i}^{\prime}$ and $w^{\prime}$. Hence by the induction hypothesis, the quantities are equal.

Let $r(w)=\#(N(w) \cup L(|w|))$. Since Lemma 11.4 shows that $r(w)$ is the maximum value of $\nu(\mathbf{i})$ as $\mathbf{i}$ ranges over $\mathcal{R}(w)$, it follows from Theorem 11.2 that $2^{r(w)-1} G_{D}(w)$ is a (symmetric) integer linear combination of the quasi-symmetric functions $K_{\Lambda}$. Thus by Theorem 3.8 of [Ste1], we obtain the following.

Corollary 11.5. For every $w \in D_{n}, 2^{r(w)-1} G_{D}(w)$ is $Q$-integral.

In other words, for $w \in D_{n}$ and strict partitions $\lambda$ of size $l=\ell(w)$, the coefficients $c_{\lambda}(w)$ appearing in the expansion

$$
2^{r(w)-1} G_{D}(w)=\sum_{\lambda} c_{\lambda}(w) Q_{\lambda}
$$

are integers. (And hence, nonnegative integers, by the work of Lam and BilleyHaiman.)

If we use (11.2) to extract the coefficient of $z_{1} \cdots z_{l}$ from $2^{r(w)-1} G_{D}(w)$, we obtain $\sum_{\lambda} c_{\lambda}(w) 2^{l} g^{\lambda}$. On the other hand, if $w$ is $V$-stable, then $\nu(\mathbf{i})=r(w)$ for every $\mathbf{i} \in \mathcal{R}(w)$, so in this case Theorem 11.2 implies

$$
2^{r(w)-1} G_{D}(w)=\sum_{\mathbf{i} \in \sigma \mathcal{R}(w)} K_{\Lambda(\mathbf{i})} .
$$

Since the coefficient of $z_{1} \cdots z_{l}$ in $K_{\Lambda}$ is $2^{l}$ (see (1.1)), it follows that in the $V$-stable case, the coefficient of $z_{1} \cdots z_{l}$ in $2^{r(w)-1} G_{D}(w)$ is $2^{l} \cdot \# \sigma \mathcal{R}(w)$. Having obtained two expressions for the coefficient of $z_{1} \cdots z_{l}$, we deduce the following.

Corollary 11.6. If $w \in D_{n}$ is $V$-stable, then the integers $c_{\lambda}(w)$ of (11.2) satisfy

$$
\# \sigma \mathcal{R}(w)=\sum_{\lambda} c_{\lambda}(w) g^{\lambda} .
$$

For example, consider the longest element $w_{0}$ of $D_{n}$. The one-line form of $w_{0}$ is $( \pm 1,-2, \ldots,-n)$, so $N\left(w_{0}\right)=\{1, \ldots, n\}, L\left(\left|w_{0}\right|\right)=\{1\}$, and $w_{0}$ is $V$-stable, by the criterion of Theorem 11.3. It is known by Corollary 5.3 of [L] or Proposition 3.16 of $[\mathrm{BH}]$ that $G_{D}\left(w_{0}\right)=P_{(2 n-2, \ldots, 4,2)}$, so $2^{r\left(w_{0}\right)-1} G_{D}\left(w_{0}\right)=Q_{(2 n-2, \ldots, 4,2)}$. In other words, there is just one term in the expansion of Corollary 11.6, yielding

$$
\# \sigma \mathcal{R}\left(w_{0}\right)=g^{(2 n-2, \ldots, 4,2)} .
$$

That is, the number of distinct reduced words for $w_{0}$ under the identification $1=\overline{1}$ is the number of standard shifted tableaux of shape $(2 n-2, \ldots, 4,2)$. This fact is proved bijectively by both Lam [L] and Billey-Haiman [BH]. 
Remark 11.7. (a) Given that $c_{\lambda}(w) \geq 0$, the same reasoning that proves Corollary 11.6 can also be used to show that for every $w \in D_{n}$, we have $\# \sigma \mathcal{R}(w) \leq$ $\sum_{\lambda} c_{\lambda}(w) g^{\lambda}$, with equality occurring if and only if $w$ is $V$-stable. By Theorems 4.18 and 4.35 of $[\mathrm{L}]$, it also follows that $\# \sigma \mathcal{R}(w)=\sum_{\lambda} b_{\lambda}(w) g^{\lambda}$ for certain nonnegative integers $b_{\lambda}(w) \leq c_{\lambda}(w)$.

(b) One might hope to prove Corollary 11.5 directly from the $P$-integrality of $G_{D}(w)$, bypassing Theorem 11.2. This would require $r(w)>\ell(\lambda)$ for every strict partition $\lambda$ such that $c_{\lambda}(w)>0$. However this fails, even in the $V$-stable case. Alternatively, one could attempt to use (8.1) to bypass Theorem 11.2; this would require $r(w)>\ell_{ \pm 1}(\mathbf{i})$ for every $\mathbf{i} \in \mathcal{R}(w)$. However again this fails, even in the $V$-stable case.

(c) A natural question to ask at this point is how the set of $V$-stable elements overlaps with the set of finely symmetric elements. By Corollary 7.2, we know that $w$ is $A$-stable if and only if $N(w) \subseteq L(|w|)$. Comparing this with Theorem 11.3, we see that the only $A$-stable members of $D_{n}$ that are also $V$-stable are those that satisfy $N(w)=L(|w|)$; by Proposition 9.8, these are the $\ell_{ \pm 1}$-invariant elements. Otherwise, if $w$ is finely symmetric but not $A$-stable, then $w \in A_{n-2} s_{1} s_{\overline{1}} A_{n-2}$ (Remark 9.5). However the criterion of Lemma 9.2(d) shows that all such elements satisfy $L(|w|)=\{1\}$, and hence are $V$-stable. But the members of $w \in A_{n-2} s_{1} s_{\overline{1}} A_{n-2}$ are also $\ell_{ \pm 1}$-invariant, so we conclude that $w$ is finely symmetric and $V$-stable if and only if $w$ is $\ell_{ \pm 1}$-invariant.

(d) We claim that $V$-stability cannot be characterized by means of pattern avoidance. Indeed, since fine symmetry does have a pattern-avoidance characterization (Theorem 9.6), a set of patterns for $V$-stability would, by the previous remark, also imply the existence of a set of patterns for $\ell_{ \pm 1}$-invariance. However the discussion prior to Proposition 9.8 shows that a set of such patterns does not exist.

Let $(2 n-1) ! !=1 \cdot 3 \cdot 5 \cdots(2 n-1)$.

Proposition 11.8. There are $2(2 n-1) ! !-2^{n-1}(n-1) ! V$-stable members of $D_{n}$.

Proof. For a given $w \in A_{n-1}$ with $l \geq 2$ left-minima, there are $2^{n-l+1}$ elements $w^{\prime} \in D_{n}$ such that $\left|w^{\prime}\right|=w$ and $L(w) \subseteq N\left(w^{\prime}\right)$. If $w$ has only one left-minimum (i.e., the first entry of $w$ is 1 ), then there are only $2^{n-1}$ such elements, not $2^{n}$. Hence by Theorem 11.3, there are

$$
\sum_{w \in A_{n-1}} 2^{n-\# L(w)+1}-\sum_{w \in A_{n-2}} 2^{n-1}
$$

$V$-stable members of $D_{n}$. Apply Lemma 2.6 with $q=1 / 2$.

\section{APPENDIX}

Tables 1 and 2 list the number of members of $B_{n}$ and $D_{n}$ (for $n \leq 7$ ) belonging to the various subsets identified in Parts I and II, respectively. (Abbreviations: $T=$ top, $B=$ bottom, $F C=$ fully commutative, $F S=$ finely symmetric, $A=A$ stable, $\ell=\ell_{ \pm 1}$-invariant, $I I=A_{n-2} D_{2} A_{n-2}$.) 
TABLE $1 . B_{n}$

\begin{tabular}{|c|c|rrrrrrr|}
\hline$X$ & $\# X_{n}$ & 1 & 2 & 3 & \multicolumn{1}{c|}{4} & \multicolumn{1}{c|}{5} & \multicolumn{1}{c|}{6} & \multicolumn{1}{c|}{7} \\
\hline All & $2^{n} n !$ & 2 & 8 & 48 & 384 & 3840 & 46080 & 645120 \\
$A$-reduced & $\frac{1}{4}(n+2) !+\frac{1}{2} n !$ & 2 & 7 & 33 & 192 & 1320 & 10440 & 93240 \\
$T, B$ & $(n+1) !$ & 2 & 6 & 24 & 120 & 720 & 5040 & 40320 \\
$T \cap B$ & $n !(1+H(n))$ & 2 & 5 & 17 & 74 & 394 & 2484 & 18108 \\
$F C$ & $(n+2) C(n)-1$ & 2 & 7 & 24 & 83 & 293 & 1055 & 3860 \\
$F C \cap T$ & $\left(\begin{array}{c}2 n \\
n\end{array}\right)$ & 2 & 6 & 20 & 70 & 252 & 924 & 3432 \\
$F C \cap B$ & $C(n)+C(n+1)-1$ & 2 & 6 & 18 & 55 & 173 & 560 & 1858 \\
$F C \cap T \cap B$ & $C(n+1)$ & 2 & 5 & 14 & 42 & 132 & 429 & 1430 \\
\hline
\end{tabular}

TABLE $2 . D_{n}$

\begin{tabular}{|c|c|rrrrrr|}
\hline$X$ & $\# X_{n}$ & 2 & 3 & \multicolumn{1}{c|}{4} & \multicolumn{1}{c|}{5} & \multicolumn{1}{c|}{6} & \multicolumn{1}{c|}{7} \\
\hline All & $2^{n-1} n !$ & 4 & 24 & 192 & 1920 & 23040 & 322560 \\
$V$-stable & $2(2 n-1) ! !-2^{n-1}(n-1) !$ & 4 & 22 & 162 & 1506 & 16950 & 224190 \\
$F S$ & $\frac{1}{2}(n+1) !+(n-1)(n-1) !$ & 4 & 16 & 78 & 456 & 3120 & 24480 \\
$A$ & $\frac{1}{2}(n+1) !$ & 3 & 12 & 60 & 360 & 2520 & 20160 \\
$\ell, I I$ & $(3 n-2)(n-1) !$ & 4 & 14 & 60 & 312 & 1920 & 13680 \\
$A \cap \ell, A \cap I I$ & $(2 n-1)(n-1) !$ & 3 & 10 & 42 & 216 & 1320 & 9360 \\
$\ell \cap I I$ & $(n-1) !(n+2 H(n-1))$ & 4 & 12 & 46 & 220 & 1268 & 8568 \\
$A \cap \ell \cap I I$ & $(n-1) !(1+2 H(n-1))$ & 3 & 8 & 28 & 124 & 668 & 4248 \\
$F C$ & $\frac{1}{2}(n+3) C(n)-1$ & 4 & 14 & 48 & 167 & 593 & 2144 \\
$F C \cap A$ & $\frac{1}{2}\left(\begin{array}{c}2 n \\
n\end{array}\right)$ & 3 & 10 & 35 & 126 & 462 & 1716 \\
$F C \cap I I$ & $3 C(n)-C(n-1)-1$ & 4 & 12 & 36 & 111 & 353 & 1154 \\
$F C \cap A \cap I I$ & $2 C(n)-C(n-1)$ & 3 & 8 & 23 & 70 & 222 & 726 \\
\hline
\end{tabular}

\section{REFERENCES}

[BH] S. Billey and M. Haiman, Schubert polynomials for the classical groups, J. Amer. Math. Soc. 8 (1995), 443-482. CMP 95:05

[BJS] S. Billey, W. Jockusch and R. Stanley, Some combinatorial properties of Schubert polynomials, J. Algebraic Combinatorics 2 (1993), 345-374. MR 94m:05197

[B] N. Bourbaki, "Groupes et Algèbres de Lie, Chp. IV-VI", Masson, Paris, 1981. MR 83g: 17001

[E] P. H. Edelman, Lexicographically first reduced words, Discrete Math. 147 (1995), 95-106. CMP 96:05

[F] C. K. Fan, "Commutative Elements of a Weyl Group”, Ph. D. thesis, MIT, 1995.

[FK1] S. V. Fomin and A. N. Kirillov, Combinatorial $B_{n}$-analogues of Schubert polynomials, Trans. Amer. Math. Soc. 348 (1996), 3591-3620. CMP 96:15

[FK2] S. V. Fomin and A. N. Kirillov, The Yang-Baxter equation, symmetric functions, and Schubert polynomials, Discrete Math. 153 (1996), 123-143. CMP 96:14

[FS] S. V. Fomin and R. P. Stanley, Schubert polynomials and the nilCoxeter algebra, Adv. in Math. 103 (1994), 196-207. MR 95f:05115

$[\mathrm{H}] \quad$ J. E. Humphreys, "Reflection Groups and Coxeter Groups", Cambridge Univ. Press, Cambridge, 1990. MR 92h:20002

[L] T. K. Lam, "B and D Analogues of Stable Schubert Polynomials and Related Insertion Algorithms", Ph. D. thesis, MIT, 1995.

[M] I. G. Macdonald, "Symmetric Functions and Hall Polynomials", Oxford Univ. Press, Oxford, 1979. MR 84g:05003

[St1] R. P. Stanley, On the number of reduced decompositions of elements of Coxeter groups, Europ. J. Combin. 5 (1984), 359-372. MR 86i:05011 
[St2] R. P. Stanley, "Enumerative Combinatorics, Vol. I," Wadsworth \& Brooks/Cole, Monterey, 1986. MR 87j:05003

[Ste1] J. R. Stembridge, Enriched P-partitions, Trans. Amer. Math. Soc. 349 (1997), 763-788.

[Ste2] J. R. Stembridge, On the fully commutative elements of Coxeter groups, J. Algebraic Combin. 5 (1996), 353-385.

Department of Mathematics, University of Michigan, Ann Arbor, Michigan 481091109 